Florida International University FIU Digital Commons

$7-31-2013$

\title{
Site Specifc Growth of Metal Catalyzed Silica Nanowires for Biological and Chemical Sensing
}

Eric G. Huey

Florida International University, ehuey001@fiu.edu

DOI: $10.25148 /$ etd.FI13120411

Follow this and additional works at: https:// digitalcommons.fiu.edu/etd

Part of the Metallurgy Commons, Nanoscience and Nanotechnology Commons, and the Nanotechnology Fabrication Commons

\section{Recommended Citation}

Huey, Eric G., "Site Specifc Growth of Metal Catalyzed Silica Nanowires for Biological and Chemical Sensing" (2013). FIU Electronic Theses and Dissertations. 984.

https://digitalcommons.fiu.edu/etd/984

This work is brought to you for free and open access by the University Graduate School at FIU Digital Commons. It has been accepted for inclusion in FIU Electronic Theses and Dissertations by an authorized administrator of FIU Digital Commons. For more information, please contact dcc@fiu.edu. 


\section{FLORIDA INTERNATIONAL UNIVERSITY}

Miami, Florida

\section{SITE SPECIFIC GROWTH OF METAL CATALYZED SILICA NANOWIRES FOR BIOLOGICAL AND CHEMICAL SENSING}

A dissertation submitted in partial fulfilment of the requirements for the degree of DOCTOR OF PHILOSOPHY

in

ELECTRICAL ENGINEERING

by

Eric G. Huey 


\section{To: Dean Amir Mirmiran}

College of Engineering and Computing

This dissertation, written by Eric G. Huey, and entitled Site Specific Growth of metal Catalyzed Silica Nanowires for Biological and Chemical Sensing, having been approved in respect to style and intellectual content, is referred to you for judgment.

We have read this dissertation and recommend that it be approved.

Sakhrat Khizroev

Nezih Pala

Kinzy Jones

Sylvia Thomas

Abdul K. Rumaiz

Shekhar Bhansali, Major Professor

Date of Defense: July 31, 2013

The dissertation of Eric G. Huey is approved.

Dean Amir Mirmiran

College of Engineering and Computing

Dean Lakshmi N. Reddi

University Graduate School

Florida International University, 2013 
(C) Copyright 2013 by Eric G. Huey All rights reserved. 


\section{ACKNOWLEDGMENTS}

During the course of my graduate studies I have been supported by numerous persons, each helpful in guiding me to this point. First, I am highly indebted to my supervisor Dr. Shekhar Bhansali, Alcatel-Lucent Professor and chair of the Electrical and Computer Engineering Department at Florida International University, for providing me this opportunity to grow as an engineer and a scientist. His patience and insight have been instrumental in developing talents and skills that will serve me for the duration of my career. His belief in my ability to think critically and produce quality work has been steadfast and unwavering. The opportunity to work beside and learn from him has allowed me to contribute to the human knowledge but also to use graduate school as vehicle to drive my success in any endeavour I choose. For this I am indebted to him for the rest of my life.

I would like to thank, Dr. Subramanian Krishnan for his contributions to my work and my development as an engineer, a scientist and as a person. His careful critiques and insights were instrumental in presenting a fresh perspective on my research to my committee and research community. Also, I am grateful to Dr. Sunil Arya, Dr. Ajeet Kaushik, and Kevin Luongo for their continuous support. Together, they motivated and inspired me to move forward to completion, in spite of experimental and administrative setbacks.

I am most appreciative to Drs. Jack Bass, William Pratt, D. Peter Siddons, and Abdul Rumaiz, for encouraging me to pursue educational training past the undergraduate level. Their lobbying on behalf on numerous occasions has made funding my graduate work possible. Without such support, my work would not have been completed.

Heartfelt thanks to Dr. Sonja Montas-Hunter, Dr. Marcus Huggans, Dr. Sylvia Thomas, Mr. Terrence Buck, and Mr. Bernard Batson. Their mentorship and guidance eased 
the internal feelings of self-defeat that clouded my thoughts and redirected my focus on pressing forward to finish my doctorate.

Thanks to my remaining committee members, Dr. Sakhat Khizroev, Dr. Nezih Pala, and Dr. Kinzy Jones, for their valuable discussions and suggestions.

I am sincerely indebted to Dr. Elhag Shaban, for introducing me to research. The opportunity to work with him was the first step that led to the writing of this dissertation. All of the success I will achieve due to the awarding of this doctorate is attributed to the opportunity he gave me, at a time when I was academically undeserving. For his kindness and belief in my work ethic I am forever appreciative and grateful.

I am grateful to Alen Jones, for reading my dissertation and the articles published from it. His encouragement and critique helped keep me motivated to finish this document. Thanks to Brandon Richard, Frank Alexander, and Justin Boone, my friends and my collaborators, for great memories and support.

Thanks to Brent Huey and Princess Huey, my brother and sister, for your support and encouragement throughout this process. To my lab mates Dr. Al-Aakhir Rogers, Dr. Dorielle Tucker-Price, Dr. Eduardo Murphy-Perez, Dr. Rudran Ratnadurai, Dr. Puneet Khanna, Aparajita Singh, Ange Marie Patricia Fievre, Syed Khalid Pasha, Michael Celestin, Abhishek Dey, Krystine Pimentel, and Nimesh Pokhrel thank you all for insights and camaraderie, during the duration of this journey. To Angela Colucci, Randy Matos ,Nicolas Norena, Celisse Zabalo, Adrian Martin, Justin Chandra-Shekhar Kalap, Andres Felipe Diaz Cruz, and Michel Rivas thank you for allowing me the privilege of mentoring each of you. I have enjoyed supervising you during my time at Florida International University and wish each of you the best as you become credentialed and more into your careers. 
I want to acknowledge my mother, Angela $\mathrm{H}$. Williams and my grandfather, the late Leo W. Huey for making me strong and loving me unconditionally. Your support has been unwavering and your belief in my ability to achieve has been steadfast, since the day I was born. I love both of you. There are not any words that I could ever write that could fully capture or describe the influence both of you have had on my life or the debt I owe to both of you for such unconditional support.

Finally, I would like to thank God, for providing me great friends and mentors throughout my life and for granting me wisdom and understanding.

Humbly,

Eric G. Huey 
ABSTRACT OF THE DISSERTATION

SITE SPECIFIC GROWTH OF METAL CATALYZED SILICA NANOWIRES FOR

BIOLOGICAL AND CHEMICAL SENSING

by

Eric G. Huey

Florida International University, (2013)

Miami, Florida

Shekhar Bhansali, Major Professor

In this research the integration of nanostructures and micro-scale devices was investigated using silica nanowires to develop a simple yet robust nanomanufacturing technique for improving the detection parameters of chemical and biological sensors. This has been achieved with the use of a dielectric barrier layer, to restrict nanowire growth to site-specific locations which has removed the need for post growth processing, by making it possible to place nanostructures on pre-pattern substrates. Nanowires were synthesized using the Vapor-Liquid-Solid growth method. Process parameters (temperature and time) and manufacturing aspects (structural integrity and biocompatibility) were investigated.

Silica nanowires were observed experimentally to determine how their physical and chemical properties could be tuned for integration into existing sensing structures. Growth kinetic experiments performed using gold and palladium catalysts at $1050{ }^{\circ} \mathrm{C}$ for 60 minutes in an open-tube furnace yielded dense and consistent silica nanowire growth. This consistent growth led to the development of growth model fitting, through use of the Maximum Likelihood Estimation (MLE) and Bayesian hierarchical modeling. Transmission electron 
microscopy studies revealed the nanowires to be amorphous and X-ray diffraction confirmed the composition to be $\mathrm{SiO}_{2}$. Silica nanowires were monitored in epithelial breast cancer media using Impedance spectroscopy, to test biocompatibility, due to potential in vivo use as a diagnostic aid. It was found that palladium catalyzed silica nanowires were toxic to breast cancer cells, however, nanowires were inert at $1 \mu \mathrm{g} / \mathrm{mL}$ concentrations.

Additionally a method for direct nanowire integration was developed that allowed for silica nanowires to be grown directly into interdigitated sensing structures. This technique eliminates the need for physical nanowire transfer thus preserving nanowire structure and performance integrity and further reduces fabrication cost. Successful nanowire integration was physically verified using Scanning electron microscopy and confirmed electrically using Electrochemical Impedance Spectroscopy of immobilized Prostate Specific Antigens (PSA).

The experiments performed above serve as a guideline to addressing the metallurgic challenges in nanoscale integration of materials with varying composition and to understanding the effects of nanomaterials on biological structures that come in contact with the human body. 


\section{TABLE OF CONTENTS}

CHAPTER

PAGE

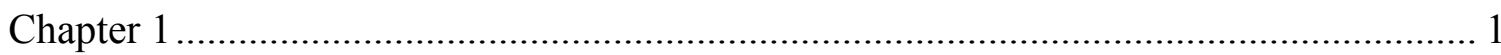

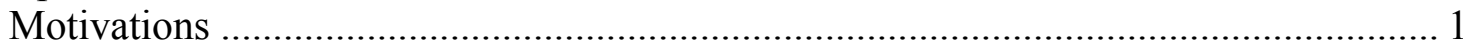

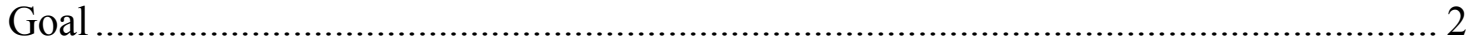

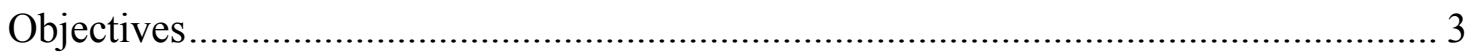

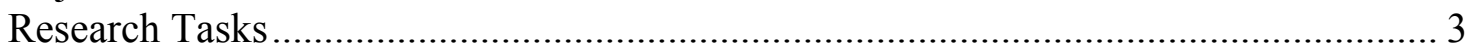

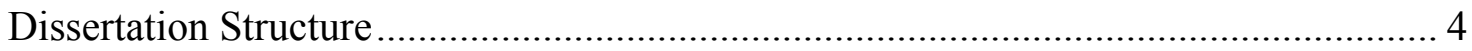

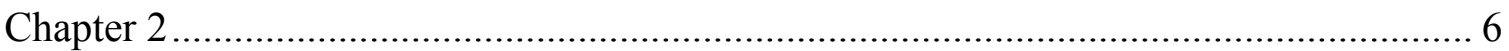

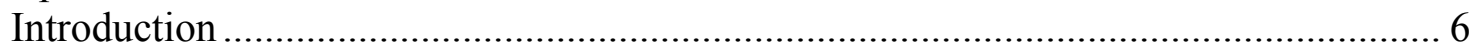

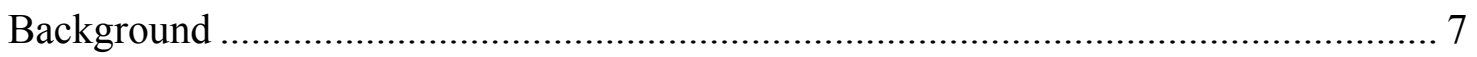

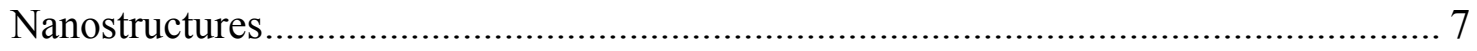

Nanowire Fabrication Techniques ………………….............................................. 8

Making the case for large scale, mass manufacturable nanowire sensors .................... 18

Advantages of General Morphology and Dimensions .............................................. 18

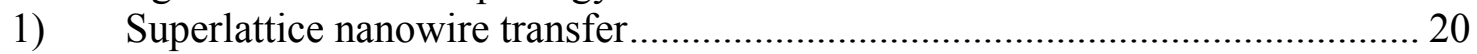

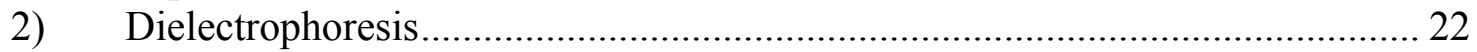

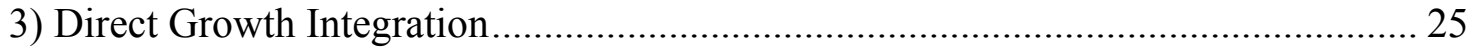

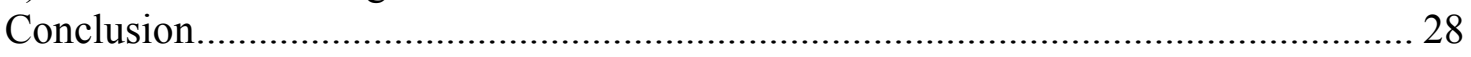

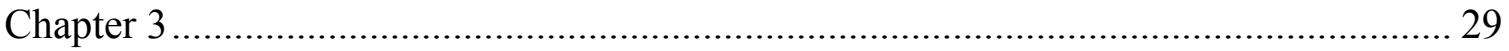

Experimental Methods and Fabrication Techniques ..................................................... 29

Experimental Methods in current research.................................................................... 34

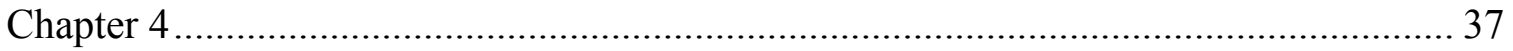

Growth Characteristics of Metal Catalyzed Silica Nanowires........................................ 37

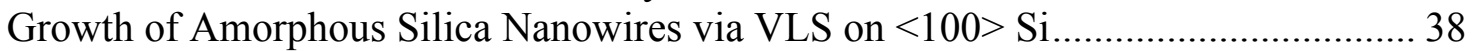

Growth Optimization of Silica nanowires catalyzed with $\mathrm{Au}$ on $<111>\ldots \ldots \ldots \ldots \ldots \ldots . . . . . . .46$

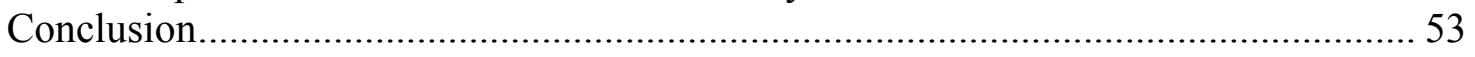

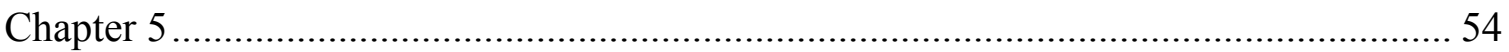

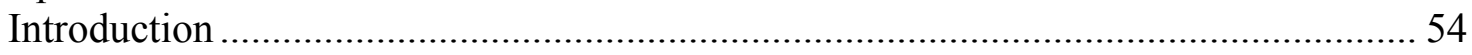

Optimized growth and integration of silica nanowires into interdigitated microelectrode

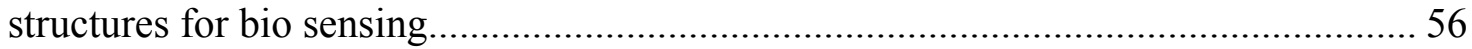

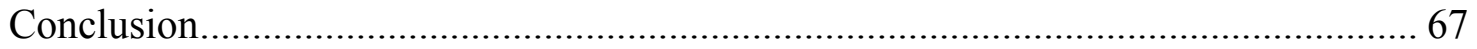

Quantitative impedance analysis of nanowires and cancer cell ..................................... 68

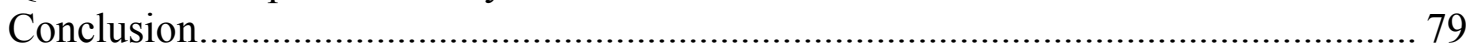

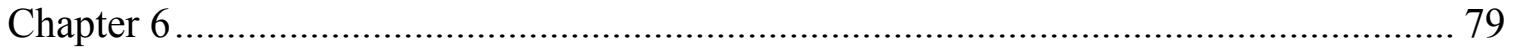

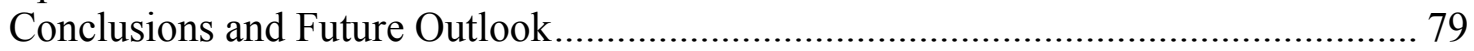

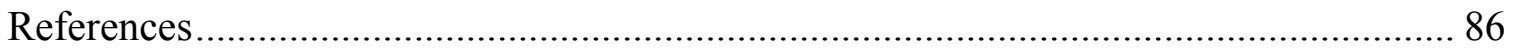




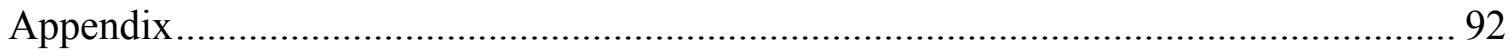

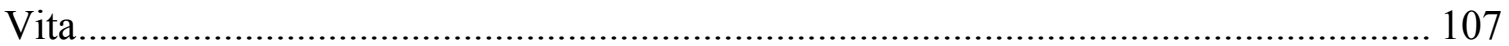




\section{Chapter 1}

In this research, the challenges of integrating nanostructures into microscale technologies were explored. The growth characteristics, cytotoxicity, and mass manufacturability of silica nanowires were investigated, to show to demonstrate the possibility of merging nanotechnology with currently used sensor platforms for commercial applications.

In this chapter, the motivation, goal and objectives of this dissertation are detailed below. Next, research tasks, significance of current research have been presented to provide justification for this research. Finally, the chapter concludes with the organization of this dissertation.

\section{Motivations}

Nanowires are foreseen as the future of many sensor systems. This is due to their high surface to volume ratios, which provide additional testing area and can lead to an increase in device sensitivity and response. Silica nanowires have commanded the attention of researchers in nanoscience due to their tunable aspect ratios, their dependent optical [3], photonic [4], and electronic properties [5] with excellent biocompatibility. Nanowire based sensors detect signals by reacting with the target material and translating the reaction into a measurable response such as impedance, voltage, or current. As these nanowires will be critical building blocks of future nanosystems, the ability to produce them at desired locations, in a controlled manner on any substrate is of great importance $[6,7]$. The primary challenge of producing these types of sensors is maintaining the functionality of the sensing material (nanowires) after final placement on a testing substrate, such as a bio-platform. 
This research has focused on the challenges of integrating silica oxide nanostructures (nanowires) into pre-existing devices, using the Vapor Liquid Solid Growth [8, 9]. The use of silicon based nanowires for device integration is advantageous due to the biocompatibility of this element with the human tissue and its similarities with currently used CMOS and MEMS sensing platforms which are currently silicon based. As such, this work focuses on bottom-up manufacturing and integration of silica nanowires with emphasis placed on growth substrate structure and response of nanowire-integrated platforms.

This technique of nanowire integration will remove the need for post growth processing by, a) minimizing structural damage from physical handling of the nanowires, and b) growing them in place on a target (final location) substrate. Additionally, this will protect the integrity of the sensing material (nanowires) and allow for more accurate testing and characterization. In future applications growing nanowires on flexible substrates will provide access to properties such as shock resistance, biocompatibility, softness and material transparency which can be taken advantage of for further experimentation and device development [10-12].

\section{Goal}

The goal of this research was to explore a simple yet robust nanomanufacturing technique for enhancing the sensitivity of chemical and biological nanosensors by integrating silica nanowires into currently used microelectrode sensing devices. Additionally, the inclusion of nano-sensing material will allow for the development of a versatile sensing structure that would able to detect both biological antigens and gas molecules, by purposeful placement of nanowire on detection surfaces. Manufacturing and placing them for 
characterization/functionality is of great importance as nanowires become more useful as sensing material in devices. The central focus of this research is to address the challenge of mass manufacturing nanowires for integration into biosensing devices by developing techniques that allow for, low cost and site-specific nanowire growth on target (working) substrates. The development of a sensor with a suitable growth substrate will offer new avenues in research and development of nanowire based sensors.

\section{Objectives}

The envisioned objective would be accomplished by-

(A) Examining the role of annealing temperature on Silica nanowire growth, to develop a growth model that will yield nanowires that are robust and of the appropriate aspect ratio (surface to volume) needed for testing,

(B) Explore the biological effects of silica nanowires to their sensing environments, to address cytotoxicity issues that may arise during device characterization and, (C) Investigating the growth mechanics of integrated silica nanowires to gain an understanding of the structural and adherence properties for testing and characterization of a functional biological and gas sensors.

\section{Research Tasks}

Accomplishment of the research objectives would involve the following research tracks; Manufacturing, Characterization, and Applications. Site specific growth of silica nanowires was pursued using a dielectric barrier layer. Also, the growth characteristics of silica nanowires were performed to study the influence of ambient and low pressure gas mixtures on the nanowire to determine a suitable growth and integration regiment. After which direct integration of silica nanostructures into microelectrode test devices occurred. 
Characterization of nanowires was performed using scanning electron microscopy, transmission electron microscopy, electron dispersive spectroscopy, to verify elemental composition. X-ray diffraction was performed to glean information concerning the nanowires surface profile. Lastly, chemical and biological sensing applications were explored. Silica nanowires catalyzed by both $\mathrm{Au}$ and $\mathrm{Pd}$ integrated into microelectrode sensing platforms were modified with PSA antigens for chemical analysis and used hydrogen testing, respectively.

\section{Significance of the current research}

Nanostructures are becoming prevalent in our everyday lives (e.g. healthcare and cosmetic products). For them to become more widely available commercially, current technology and nanotechnology must be merged in a way that uses the best of both to enhance human life. However for this to occur the challenges of integration to make such devices must explored and understood.

\section{Dissertation Structure}

In this thesis, metal oxide nanowires have been produced using a bottom-up Vapor Liquid Solid mechanism and have been shown to be experimentally biocompatible and integratable into microelectrode sensing structures.

Chapter 2 reviews the various types of nanowire fabrication methods, and discusses the process parameters of nanowire synthesis for each. Additionally, the chapter discusses the advantages of large scale manufacturing of nanowire bases sensors, outlining the electrical transport properties and the ease of functionalization of such an existing device. Then, current state of the art nanowire integration techniques are discussed highlighting strengths and weaknesses for current technologies. Finally, a discussion on the issues preventing 
integratable nanowire based structure from being widely available commercially. The limits on such device and how current technology can insure these new nano integrated structures can be preserved.

Chapter 3 presents a brief review of the experimental methods use to collect data for the experiments discussed in this dissertation. Included are some basic theoretical principles of operation on devices such scanning electron microscopy (SEM), transmission electron microscopy (TEM), X-ray and diffraction spectroscopy (XRD) and a brief overview of the fabrication process used for device development, nanowire integration and device measurement.

Chapter 4 presents discussion on the growth and morphology of silica nanowires at $1050^{\circ} \mathrm{C}$ and $1150^{\circ} \mathrm{C}$ and with explanation on high temperature growth inhibition and how taking advantage of such properties could allow for successful integration of nanowires into pre-existing silicon based structures.

Chapter 5 presents details on the integration of silica nanowire via $\mathrm{SiO} 2$ barrier layer. Additionally, functionalization of these wires for PSA detection is demonstrated and explained. Also, data is presented address the cytoxiciyt of silica nanowire to their sensing environment and other biological media, specifically, epithelial breast cancer cells.

Chapter 6 summarizes the findings of this work based on the goals, motivations and research objectives reported above and present details of future work and research avenues that can and are being undertaken to further this work such as low temperature growth, vertical nanowire growth and integration into more complex complementary metal oxide semiconductor structures. 


\section{Chapter 2}

\section{Introduction}

For years nanostructures have emerged as a source of new innovation. Their physical properties such as length, width, and high aspect structures provide the potential for applications in an arena of situations. More so their physical structure also lends them to be chemically altered for applications involving biological and chemical applications. Researchers have working for years to discover and develop ways of using nanostructures to serve better human causes in industries such as health care, defense, and environmental monitoring. With these advancements the advent of nanostructure influences in our daily lives will be a reality soon. Innovations and ideas have to be vetted thoroughly to insure that theoretical calculations and hypothesis are practical to integrate into society. To be practical ideas/innovations will need to be marketable and mass manufacturable and commercialized for consumer purchase. To achieve these goals work should be pursued with the focus on integrating nanostructures into existing detection platforms and devices.

Nanostructures such as nanowires are on the precipice of being introduced into prepackage devices. These devices will provide services that will allow for more accurate testing of human and biological materials. For this transition from lab only phase into the market for profitability and human use nanostructures must be produced packaged in format that allows for their repeatable use in atmosphere not as selectively controlled as a laboratory. This chapter introduces nanowires, specifically their discovery, current methods of fabrication, then addresses issues concerning manufacturing and electron transport, then present techniques for device integration and finally a discussion on issues preventing the production of devices with nanowires incorporated into their structure. 


\section{Background Nanostructures}

Nanostructures are defined as particles or design constructs with dimensions below $100 \mathrm{~nm}$. Nanostructures such as nanotubes and nanowires are generally one dimensional structure with high aspect ratios, one of their unique qualities. These structures don't exist naturally and have to be synthesized or grown using methods developed in laboratory settings.

\section{Nanotubes}

Carbon nanotubes (CNT's) are nanostructures with dimensions ranging lengthwise up hundreds of microns and with diameters that are nanometers in scale. These structures are colloquially described as honeycomb lattices rolled into cylinders[13]. Carbon nanotubes are significant due to unique electrical properties that are tailorable by adjustment of nanotube geometry. After their discovery by Ijima[14] it was widely believed that the carbon nanotubes would provide new insights into condensed matter physics and that their development would allow researchers to contribute to enhancing human life through innovative applications. During the past twenty years, carbon nanotubes have been demonstrated as diodes, transistors, gas sensors, gas separators, gas and energy storage applications[15]. Additionally, they have found use in metrology, as cantilever tips in high resolution atomic force microscopy (AFM), for mapping trenches and holes in IC's [16]. However, carbon nanotubes are limited in their use due to the issues with inflexible geometries, which makes tailoring and surface modification difficult. Also, hydrogen's

contribution to the growth process is yet to be explored, as well as issues with alignment and surface disorders in synthesized nanotubes. To address these concerns another nanostructure, 
the nanowire has garnered interest among researchers who believe its characteristics will address the drawbacks of CNT's.

\section{Nanowires}

Nanowires are defined as nanostructures that can be composed of both metallic and nonmetallic elements with nanometer sized diameters and micron long lengths[17]. These types of nanostructures are robust and have physical strength on the order of 1 million psi; this strength is directly attributed to their crystalline structure and high aspect ratio dimensions. Nanowires were originally called nanowhiskers due to their whisker-like appearance. Nanowires were first reported 1964 by Wagner and Ellis[8] using the Vapor Liquid Solid(VLS) growth mechanism, which will described in more detail in a later section. Since their discovery, new methods of nanowire synthesis have been explored and developed. These include the hydrothermal method Oxide Assisted Growth mechanism and the Vapor Liquid Solid mechanism. These methods yield appreciable and dense wires with their own unique morphologies. A description of each is presented below with examples of that demonstrate the novelty and advance each technique has contributed to the state of the art.

\section{Nanowire Fabrication Techniques}

\section{Hydrothermal}

The hydrothermal growth process is use to synthesize of nanostructures such as nanorods (figure 1) and nanowires of defined morphology and compositions. This method produces these structures with by of reduction and oxidation reactions between precursors/catalyst involved in growth process. Using these reactions has the added benefit of allowing the 
hydrothermal method to occur at lower temperatures than those processes that will be discussed below.

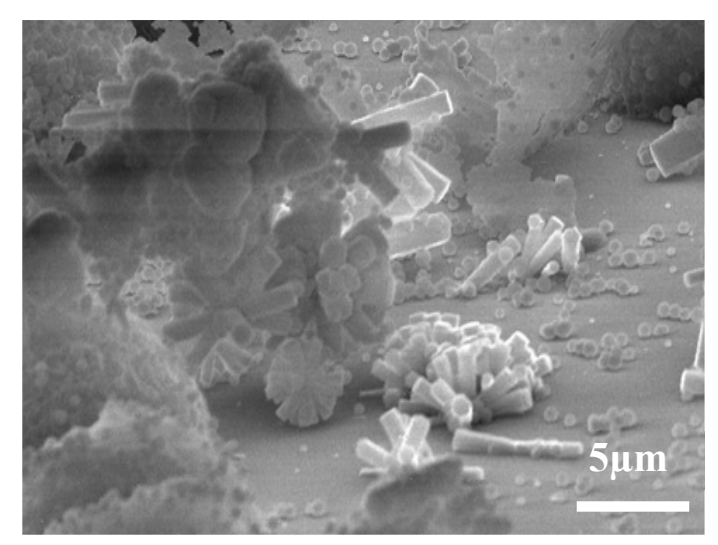

Figure 1. Zinc oxide nanorods. [Courtesy Eric Huey]

Popular among researchers for nanowire fabrication is zinc oxide. B. Liu et al chose $\mathrm{ZnO}$ as a base material to develop nanorods. $\mathrm{ZnO}$ wide band gap of $3.37 \mathrm{eV}$ along with its demonstrated lasing capabilities at room temperature have made it a material nanostructure development. [18] Zinc Oxide nanorods were synthesized using a novel wet chemical method to yield structures with aspect ratios between $30-40$ and diameters of $50 \mathrm{~nm}$. A solution containing zinc nitrate, $\mathrm{NaOH}$ in de-ionized water was mixed with pure alcohol and ethlyendediamine and were autoclaved and placed into an electric oven set to $180^{\circ} \mathrm{C}$ for 20hrs. Harvested wires were observed to be uniform in diameter and growth along the c-axis was maintained along the length of the wires. The diffraction pattern of the wires confirmed a $\mathrm{Zn}: \mathrm{O} \approx 1: 1$. The success of this process provided an experimental foundation that other researchers would use to develop tailored recipe's for the hydrothermal growth metal or metal-oxide based nanostructures. Similarly, Wang et al synthesized single crystalline $\mathrm{Zn}$ based nanowires using a simple hydrothermal method. Nanowire/whiskers with diameters of 
50-80nm and lengths 6um were produced. The method used involved polethylen glycol, distilled water and PEG solution and was described as "simple" by authors due the non requirement of catalysts or complex apparatus'. Additionally, the fabricated wires showed UV emission peaks at $392 \mathrm{~nm}$ at room temperature with a XE lamp excitation source demonstrating possible use in optical applications. [19]

Jiaqiang et. al synthesized Zinc nanorods for gas sensing. Using cetyltrimethylammonium bromide $(\mathrm{CTAB})$ and zinc powder wires were produced at $182^{\circ} \mathrm{C}$ [20] The nanorods were $40-80 \mathrm{~nm}$ in diameter with lengths of a approximately a $1 \mathrm{um}$. The wires were thermally resilient up to $600^{\circ} \mathrm{C}$; however nanorod diameters increased to $100 \mathrm{~nm}$ with prolonged heating. Additionally, the zinc nanorods were tested for gas sensing with $\mathrm{C}_{2} \mathrm{H}_{5} \mathrm{OH}, \mathrm{HCHO}, \mathrm{NH}_{3}$, and LPG, 90\# gasoline. For optimal results the working temperature of the nanorod sensors was adjusted periodically during experimentation. The sensors demonstrated the highest sensitivity to LPG at 500ppm. For additional testing the nanorods were doped with $\mathrm{PdO}$ to enhance sensing. The $\mathrm{PdO}$ when reduced provides electrons back to the oxide, increasing sensor response times to less than 30seconds.

Transition metals are also able to be used in this method for nanowire synthesis. The unfilled octets of the below metals makes them readily available to the reduction and oxidation events needed to for nanowire formation. Liu et al used copper II glycerol reduction to synthesis nanowires with diameters averaging $85 \mathrm{~nm}$ and with lengths of several microns [21]. The novelty and versatility of process lies in the $120^{\circ} \mathrm{C}$ temperature that the wires were synthesized at. Additionally, the wires were of uniform morphology due to the contributions of $\mathrm{NaOH}$ and glycerol in the reduction of copper. Specifically, the mixture of glycerol and $\mathrm{NaOH}$ created a high viscosity fluid which prohibited copper particles from 
agglomerating, thus allowing stable suspension in fluid needed for uniform wire growth. Lastly the absorption spectrum of the wires showed peaks at $590 \mathrm{~nm}$ in agreement with previous literature. It was also noted that oxide formation on the wires during synthesis did not reveal any noticeable affects during UV characterization.

Lin et al [22] demonstrated the growth the silicon oxide nanowires via water assisted synthesis. Synthesized wires were several microns long with diameters ranging from tens to hundred of nanometers in length. Scanning Electron Microscopy (SEM) revealed the nanowires to be smooth and straight with uniform diameters and length. Additionally, chemical bonding studies using Fourier Transform Infrared Spectroscopy FTIR revealed that lattice vibrations were noticeable and were attributed to the $\mathrm{Si}-\mathrm{OH}$ groups on the wire surface. This is also attributed to the smooth surface of the wire in that $\mathrm{OH}-$ and $\mathrm{H}+$ block defects from forming on the wire surface. The authors stressed the novelty of the process by providing clear distinction between this growth method and the Oxide Assisted Growth (OAG) process. The OH- and $\mathrm{H}$ not only prevent defects on surface but are also involved in the wire formation under supercritical hydrothermal conditions with during sample preparation between $400-470{ }^{\circ} \mathrm{C}$ at $6-10 \mathrm{MPa}$ for 20 hours.

Xie et al [23] produced cobalt nanowires via hydrothermal reduction of cobalt (II) citrate by hypophosphite at $160^{\circ} \mathrm{C}$. Nanowires had lengths up to several hundred microns and diameters between $150-330 \mathrm{~nm}$. Citrate salts were used during synthesis to control the morphology of the wires. Additionally, the wires show ferromagnetism with greater coercively than similar bulk materials. The authors employ that this method is environmentally friendly synthetic route for the fabrication of 1-deminstional nanostructures. 
Lastly, characteristic peaks due to cobalt oxides or hydroxides were noticed during XRD characterization.

Wang et al. investigated the hydrothermal formation of alpha and beta manganese oxide (MnO)nanowires [24] This was done with initial intent that 1-D MnO nanostructures would assist in the detecting the theoretical limits of Lithium ion battery based technologies. Using a low temperature $\left(120^{\circ} \mathrm{C}\right)$ method to oxidize $\mathrm{MnO}^{2}+$ by $\mathrm{S}_{2} \mathrm{O}_{8}{ }^{2-}$ oxide nanowires wires with dimensions between $40-100 \mathrm{~nm}$ and lengths ranging 2.5 and 4.0 microns were observed. The outcomes of this work demonstrated that oxides such as $\mathrm{Zno}, \mathrm{TiO}_{2}$, could be formed readily via hydrothermal synthesis. Using proper controls and chemical reactions the method could lend itself to yield nanostructures free of catalytic impurities and a method that would that would introduce savings by reduction of production cost.

Wang et al have used the hydrothermal process for large scale production of silver nanowires by reducing silver chloride with glucose.[25] Nanowires were up to 500um in length and $100 \mathrm{~nm}$ in diameter. The wires were characterized using SEM and XRD techniques. Using SEM nanowire morphology was observed to be smooth, however small quantities of silver nano particles were observed. Nanowires also grew perpendicular to the silver Ag (200) planes. This was confirmed with HRTEM. Comparative experiments were carried out to determine optimal growth conditions for sliver nanowires. It was observed that wire growth was halted at temperatures below $140^{\circ} \mathrm{C}$; due to the inability of glucose to reduce silver chloride. At $160^{\circ} \mathrm{C}$ the reaction was possible and was presented as such in the paper. At $180^{\circ} \mathrm{C}$ the reaction and growth of wires was much faster. Other experiments were performed with other Ag based agents such as silver nitrate, and other reducing agents such as $\mathrm{OH},-\mathrm{Br}-$, and $\mathrm{Cl}-$. Observations revealed that silver nitrate reduced too quickly to form 
wires and nano particles were the product. Silver bromide and silver hydride reduce quickly to form nanowires.

The hydrothermal method is generally effective and highly repeatable, yielding nanowires of consist length and diameter. The process, as discussed is environmentally friendly due to non use of catalyst and surfactants, the use of this method for nanowire integration and growth into CMOS or pre existing platforms would introduce difficulties that could impede device function. For instance, the hydrothermal method by definition is water based; this would introduce moisture into a sensing device/platform. Moisture and water vapor have been observed to introduce artifacts and hamper sensor performance. For CMOS devices the water vapor/moisture could cause additional oxidation, thus augmenting a device structure's performance characteristics.[26]

\section{a. OAG}

Oxide assisted growth $(\mathrm{OAG})$ is a mechanism of nanostructure formation that occurs without the use of metallic catalyst, which puts it in direct contrast with the VLS method in the next section. OAG has been used to synthesize $\mathrm{Si}$ and Ge nanowires[27]. It is a preferred method of growth because it yields dense bushes of wires without the contamination issues that occur when metallic catalysts are used and competes with hydrothermal method by not using water in its synthesis process. Below, arsenic, gallium, and silicon are presented as an example of morphology and composition of wire fabricated using OAG standards.

Shi et al,[28] claimed to have grown gallium arsenide nanowires via an oxide assisted growth method. The novelty of this method is that it produces nanowires without metal catalyst. The structures produced in this study were composed of crystalline cores, $60 \mathrm{~nm}$ in diameter, covered by an additional $5 \mathrm{~nm}$ amorphous oxide. The wires were formed 
thru a decomposition of GaAs into its separate components via laser ablation, Hydrogen carrier gas was used to transport volatile $\mathrm{GaO}_{2}$ and As to the low temperature zone of the growth furnace where decomposition and nanowire growth was able occurred.

Zhang et al.[27] on a review of their work report on the distinct difference between the OAG method and VLS method. The repot finds that Si nanowire can be obtained by laser ablation without catalyst use and that wires using this method can be produced at an enhanced growth rate specifically up to 30 times higher than complimentary reactions with Fe catalyst. Additionally, the wires with diameters approaching 1nm can be synthesized with addition growth directions of 112110 , which is in direct contrast with the $<111>$ oriented growth that is dominant in VLS grown wires. Lastly, the method can also be used to synthesize wires from other group III-V materials, by using the co-existent properties of VLS and $\mathrm{OAG}$.

Nanowires synthesized with this method are without metallic impurities. This alone makes them attractive for application where such contaminates are not preferred, however due to the high temperature environments needed to produce these structures OAG is not preferred for nanowire growth method needed to accomplish the task set forth by this document. Additionally, it is possible for the OAG synthesis temperatures to exceed the thermal budgets of the device platforms of interest to this work.

\section{b. VLS}

The Vapor Liquid Solid (VLS) growth method produces nanowires via substrate vapor and metallic alloys. To produce nanowires with VLS, a Si substrate is deposited with a thin film of metal, usually a metal that has a eutectic relationship with silicon e.g. $\mathrm{Au}[29]$, $\operatorname{Pd}[30], \operatorname{Pt}[31]$. This deposited substrate is then annealed to synthesize nanowires. For 
example, Au deposited silicon substrate produces nanowires by forming eutectic alloy with Si at $363^{\circ} \mathrm{C}$. At this mark Au nucleates globules on the Si wafer surface. The size of the globules are governed by thickness of the film deposited [8, 17, 32] This Au nucleation sites remove $\mathrm{Si}$ from substrate drawing it into the gold globule. Simultaneously, Si vapor is evaporated from wafer surface and blown over the globules by argon carrier gas flowed thru heating furnace. This Si in combination with oxygen present in the ambient and native oxygen (Si wafer) are then absorbs in the Au globule. At a thermodynamic point known as supersaturation, the globule expels the $\mathrm{Si}$, either below or above the surface. This continuous expulsion forms the stems of the nanowire. Usually this growth is vertical and away from the substrate but can be aligned perpendicular or angled depending on growth parameters or crystallography of the surface of the Si substrate. These grown nanowires continue to grow consuming the gold catalyst for a period before slowing in growth when the au catalyst has been exhausted. This type of growth yields nanowires in as little as five minutes and has been demonstrated with a range of materials, with focus on process temperature and pressure, wire morphology(diameter), and optical properties.

Silicon carbide nanowires were fabricated by Wang et al[33] using vertically stored graphic powered placed in a furnace and heated to a $1550^{\circ} \mathrm{C}$, using VLS and further demonstrated that wire growth was strongly related to the pressure variation rate of the source species. Fabricated wires ranged in shape from conical to spindle shaped. Wire morphologies were controlled by varying the carrier gas for the silicon. At certain intervals the carrier gas was flowed in high quantity of gas to provide more Si for the globule thus increasing the diameter thickness of the nanowire. And at other times carrier gas pressure was lowered and a resultant shrink in diameter thickness was noticed[33]. 
Kim et al report the heating of Pd coated Si substrates for synthesis of silica oxide nanowires. Wires were grown after a wafer deposited with $5 \mathrm{~nm}$ of palladium was placed the center of a heating furnace and heated to $1000{ }^{\circ} \mathrm{C}$ for two hours in the presence of 3.1 percent oxygen and a balance of argon with a pressure of $300 \mathrm{mTorr}$. The wires were of amorphous structure with diameters between 15-100nm. An extended VLS mechanism is suggested as the primary contributor to the growth of these wires. Photoluminescence measures showed blue emission bands with peaking at 430 and $537 \mathrm{~nm}$.[34]

$\mathrm{Hu}$ et al used a method of growth that allows for the synthesis of silica nanowires without metallic catalyst. The method was discovered during a high temperature annealing and subsequent thermal oxidation of SI wafers, inside an alumina tube of $42 \mathrm{~mm}$ diameter and $80 \mathrm{~cm}$ length. A polished Si strip $<100>$ orientation was place inside a furnace tube and placed into vacuum at $6 \times 10^{\wedge}-2$ Torr and then heated to $800^{\circ} \mathrm{C}$ at $10^{\circ} \mathrm{C} / \mathrm{min}$ rate. Further heating continued to $1300^{\circ} \mathrm{C}$ and held constant for 5 hours for nanowire growth to be induced. Additionally, high purity hydrogen was flowed into the chamber at $50 \mathrm{sccm}$ at 300 Torr. Scanning Electron Microscopy (SEM) revealed the wires to be of silica composition, highly pure with no metal catalyst present. Diameters of $50 \mathrm{~nm}$ were measured, a few being thin as $5-10 \mathrm{~nm}$. Room temperature photoluminescence of the wires showed a strong emission peak at $540 \mathrm{~nm}[35]$.

Yang et al performed TEM in situ growth of VLS grown nanowires using Au catalyst and identified VLS growth in three phases, 1) alloying process, 2) nucleation, and 3) axial growth. The work was performed to gain a better understanding of growth mechanism to determine the vapor phase contribution to growth. The Au clusters had average sizes of 20.2 
+ or $-3.1 \mathrm{~nm}$. the wires were highly crystalline and generally had a $<111>$ growth direction.[9]

The VLS growth mechanism can also influenced by the use of laser ablation cluster formation. Morales et al [36] using chemical vapor deposition, was able produce this technique to grow uniform $\mathrm{Si}$ nanowires with diameters 6-20 $\mathrm{nm}$ with lengths up to 30 microns. Additionally, this method was used to synthesize wires with a multiple of metal catalysts verifying its establishing its reliability as a method of cluster formation for VLS growth.

Chang et al. have synthesized $\mathrm{ZnO}$ nanowires via the Vapor liquid solid growth mechanism using CVD methods using a three zone furnace [37]. To add novelty to the technique the researchers inserted a quartz tube into the furnace, which the samples for nanowire growth were placed. This led to the formation of $\mathrm{h}$ nanowires with high carrier concentrations without the use of dopants. This was accomplished by creating a high concentration of zinc vapor inside smaller quartz tube, which when combined with low concentrations of oxygen in a temperature controlled method led to the formation of nanostructures that were randomly oriented. TEM revealed that the wires were of varying diameters between 30 to $45 \mathrm{~nm}$ and that they were of single crystalline structure. Electrical conductivities studies revealed that while the carrier concentrations were higher due to non dopant impurities, the carrier mobility was lower, which was suggested to be due to increased scattering from lattice defects and electron-electron interactions. Generally, electron scattering occurs when electron flow is disturbed, usually a deflection of electron path.[38] This deflection reduces the speed of electrons which directly results in loss of conductivity for device that rely such performance such as Integrated circuits and transistors. 
Sekhar et al produced silica nanowires using the VLS method on deposited silicon wafers using a platinum catalyst. It was found that platinum silicide acts to facilitate bottom-up formation of silica nanowires. Briefly, prime grade 2-inch wafer were sputter deposited with various thickness of $\mathrm{Pt}(2,3,5,10$, and $100 \mathrm{~nm})$. Argon was flowed at $30 \mathrm{sccm}$ rate. The samples were introduced into a tube-furnace and heated to $1100^{\circ} \mathrm{C}$. Photoluminescence emissions were blue at $430 \mathrm{~nm}$ under excitation. Wires grown with a Pt catalyst had a thickness of $60-220 \mathrm{~nm}$ with varying diameters due to Oswald ripening, the average was $146 \mathrm{~nm}[39]$.

\section{Making the case for large scale, mass manufacturable nanowire sensors}

Advantages of General Morphology and Dimensions

Electrical Transport Properties

The properties the make nanowires attractive such as, larger surface to volume (high aspect ratio), and large absorption area also affect their electrical transport properties. The transport of electrons through nanowires is of great importance for using them for sensing. It is this transport of electrons that allows for signal detection from the environment or media where nanowires are present.

In nanowires, the resistance of the wire is governed to an extent by the bulk material, from which it is fabricated. However, at smaller scales properties such as, wire diameter, wire morphology, crystal structure, and crystal orientation become factors in the carrier transportation. For example, $\mathrm{Cu}$ is a metal that is commonly used in household and electronic wiring and displays ohmic characteristics at room temperature. Sarkar et al [40] while studying nanowires for use in oxide templates found that $\mathrm{Cu}$ displayed similar characteristics 
at 4.2.K to studies performed at room temperature. However, when $\mathrm{Cu}$ was oxidized into $\mathrm{Cu}_{2} \mathrm{O}$ (which is semi conductive) the nanowire when placed and biased between two electrodes formed two Schottky diodes. This phenomenon supports the need for understanding wire transport and shows that temperature and physical augmentation can affect electron conduction thru materials at different areas based on preparation of material.

In bulk material, the primary restriction in electron flow is lattice structure, which can cause scattering. Electron scattering is directly related to the resistance, or an objects ability to resist current flow, or electron movement. At macro scale this makes many certain materials not useful in applications needed for current sourcing. However, at the nanoscale, scattering can be avoided by the use of a property known as ballistic transport. Ballistic transport occurs when the means free path of an electron is longer than the nanostructure or device it is passing through. For nanowires this is advantageous and can normally be observed happening at the nanowire junctions. Electrical conductance in this case is governed the universal conductance, $2 \mathrm{e}^{2} / \mathrm{h}$. specifically, in the case of the metallic nanowires this electrical phenomena occurs when the Fermi wavelength of the processed (synthesized) metal has identical (similar) length as the diameter of the wire[13].

\section{Nanowire integration into sensing structures}

In order to make use of nanowires and their unique morphologies, techniques of integration have been explored. These methods seek to place nanowires into patterns and positions that would make them useful in devices outside of laboratory settings. As suitable method of nanowire integration into microscale devices would, 1) Maintaining physical integrity of nanosensing material (nanowires), by preventing the physical handling/placement of wires, 2) use materials combinations that would not compromise the thermal budgets of 
pre-existing device, and 3) produce nanowires with size control of wires with consistent and reproducible morphology. Each of the techniques below works to address the above issues.

\section{1) Superlattice nanowire transfer}

Superlattice nanowire transfer patterning (SNAP) is a technique that has been used to produce nanowires with from thin films templates[41, 42]. This technique is capable of creating nanowires with dimensions between 7 to $9 \mathrm{~nm} \pm 2 \mathrm{~nm}$. The method of transfer to final working/test substrate is robust and yields arrays of ordered and morphologically identical nanowires. The SNAP method works by patterning nanowires in plane on silicon-on-oxide (SOI) wafers and then transferring them using a poly dimethylsiloxane (PDMS) sheet. Specifically, nanowires are formed from a patterned thin film that has been deposited on the SOI wafer. Next, the substrate is placed in Hydrofluoric acid (HF) briefly to release the nanowire thin film and then a PDMS sheet is placed attached to the wires and used to peel them from the SOI wafer. The PDMS sheet containing the nanowires is then placed on a warm epoxy sheet, after conformal contact the epoxy is heated and the PDMS is carefully removed revealing aligned in plane with surface nanowires. Figure 2 illustrates an overview of the SNAP process. 

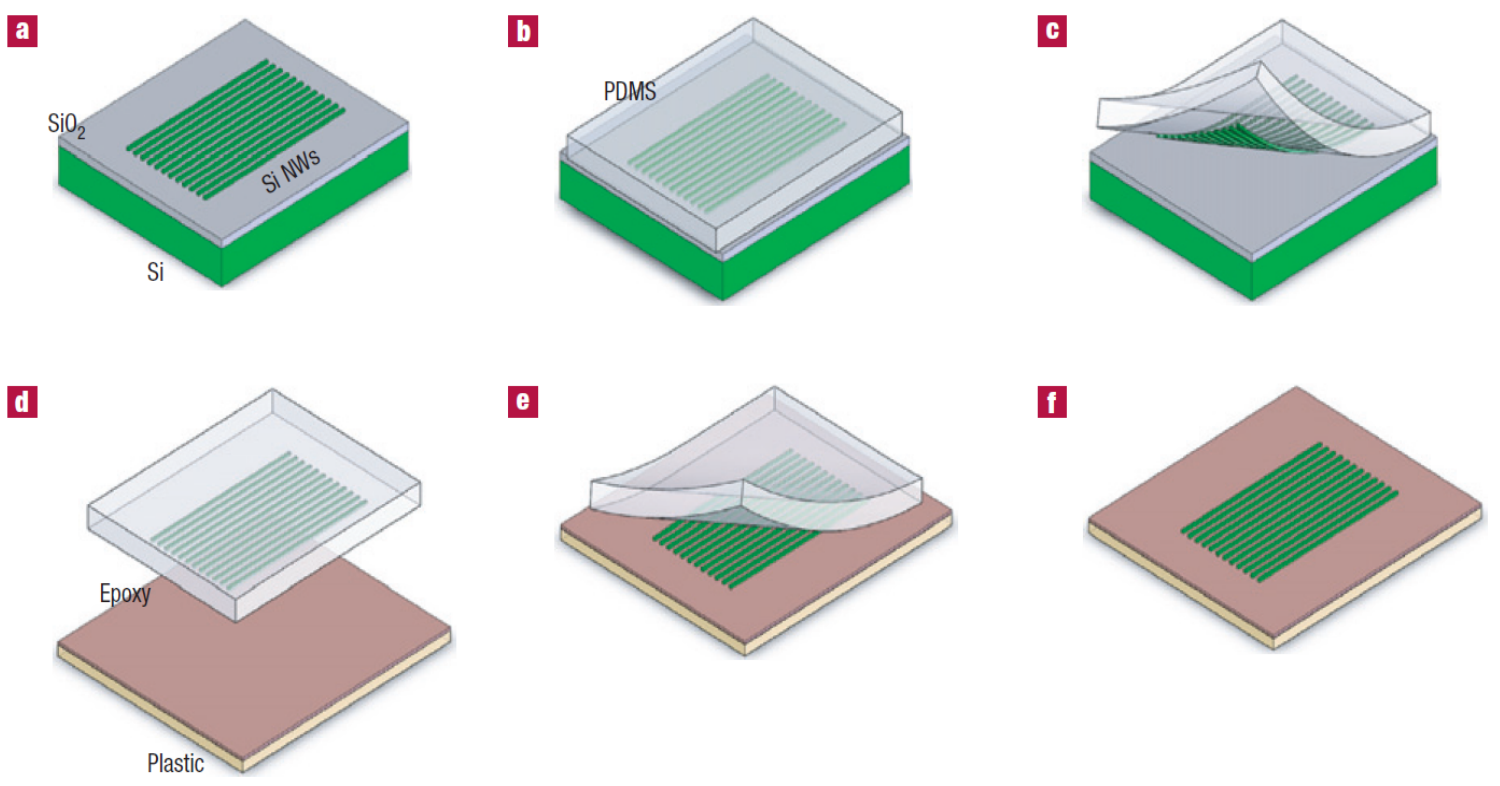

Figure 2: Illustration of the steps for transfer printing SNAP nanowires onto plastic substrates. A. Nanowires are etched into single-crystal silicon-on-insulator substrate. B. The exposed oxide is etched and a piece of PDMS makes conformal contact with the nanowire surfaces. $\mathbf{C}$. The PDMS with adhered nanowires is peeled back from the host substrate. D. A plastic substrate is spin-cast with epoxy. E. The PDMS makes conformal contact with the plastic, and the epoxy is cured. F. Peeling back the PDMS leaves behind the SNAP nanowires in their original orientation, but on plastic.(Reproduced with Permission from [6]).

The prepared nanowires obtain most of their electrical properties from the thin films from which they are patterned from. Since doping at the nanoscale can result in devices with non statistical and unpredictable behaviour[43], this type of doping is one of the advantages of this process, wires and can be tailored chemically in the prefabrication phase by doping the bulk material instead of the post process nanowires. Melosh et al[44], using earlier form of the SNAP method, that used stamps or templates, was able to synthesize nanowires on the order of $8 \mathrm{~nm}$ with matching boron and arsenic doping levels near $10^{18} / \mathrm{cm}^{3}$. As a result, this 
has led to the more recent use of the SNAP technique in the development flexible nanowire based sensors demonstrated by McAlpine et al [6, 7].

The SNAP technique has been a useful tool in the evolution of nanowire based electronic and device integration science. This has been accomplished using a template transfer method to yields wires that are aligned and preformed. However, this technique has a major drawback in the area of material handling. The PDMS transfer process involves the physical handling of nanostructures, which could result in the loss of material through breakage from PDMS attachment, heating and subsequent removal, and transfer through the lab atmosphere. Lee et al [42] demonstrated a nanowire transfer method that allows for nanowire patterning without the use of SOI wafers. Nanowires with diameters of $20-200 \mathrm{~nm}$ and lengths of 5 to $100 \mu \mathrm{m}$ were fabricated as freestanding bridges using controlled micromachining processes. Back gated and top gate p-channel field effect transistors were demonstrated with varying results. As made top gated devices work better than p-channel devices due to lack of thermal heating need to properly diffuse dopants into surface. This annealing would have damage the as prepared nanowire assembly, so the annealing did not take place. As such, the contact were masked and deposited and measurement proceeded without annealing.

\section{2) Dielectrophoresis}

The movement of particles via electrical or magnetic fields, in liquid media, is the next step from nanowire pattern transfer. This movement allows for their manipulation of without physical handling exposure to prolonged heat treatment. This is an advantage in the placement of cells, cellular components, and marker particles, which are affected by unnatural temperature fluxes[45]. Another advantage, is that particles don't need not be 
charged, since particles display electrophoretic property in the presence of electric fields [46]. In addition to being drifted through media randomly, particles can be arranged, separated, and concentrated (localized) by manipulation of external ac forces. Dielectrophoresis (DEP) is process at involved in the movement of charge particles via dipole moments.

Dielectrophoresis is a versatile tool that allows for the manipulation of nanoscale materials such as single and multi wall carbon nanotubes[47, 48]. This type of deposition involves the application of non-uniform electric fields with mild voltages, thus making it useful in wide range of applications that involve the micro assemble of nano structures or nano materials (figure 3) [46, 49].

1)

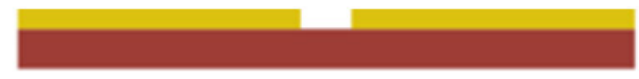

2)

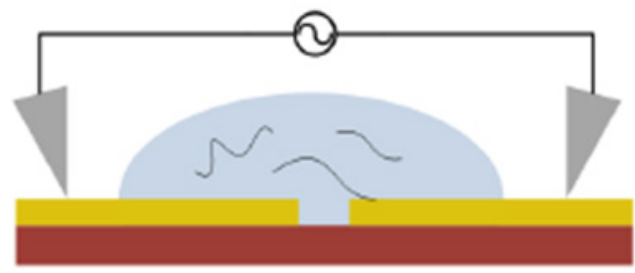

3)

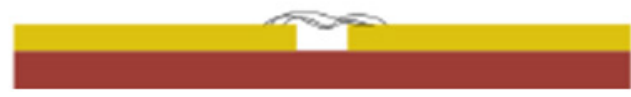

Figure 3. Process to create micro-assembly sensors Gold electrodes patterned on silicon (1) are submerged in a dispersion of either SWNT's' or RGO. An AC voltage is applied (2), and the drop is blown off to produce micro-assemblies [Courtesy MacNaughton IEEE 2010]

When a particle is place in an electric field, its experiences forces applied by that nonuniform field, depending on the material whichever pole is experiencing the strongest force will dominate and the particle will move in that direction. This process works using both AC and DC field. This is due to the field gradient effects on the particles and is governed by the 
simple for of time averaged DEP and the Classius-Mossotti function for homogenous sphere surrounded by a conducting liquid medium $[45,50]$,

$$
\left\langle F_{D E P}\right\rangle=2 \pi r^{3} \varepsilon_{m} \operatorname{Re}\left\langle\frac{\varepsilon *_{p}-\varepsilon *_{m}}{\varepsilon *_{p}-2 \varepsilon *_{m}}\right\rangle \nabla\left|\overrightarrow{E_{r m s}}\right|^{2}
$$

where $r$ the radius of the sphere is, $\varepsilon *_{p}$ is the complex permittivity of the sphere in the medium, and $\varepsilon *_{m}$ is the complex permittivity of the medium. Additionally, varying the AC frequency can be used as a tailoring mechanism for sorting of particles of specific resonances and determining electrical property attributes [51].

Using DEP Pohl et al [51] selectively separated live yeast cells from dead yeast cells, while maintaining the viability of the live cells. Yeast cells were chosen due to their ability to survive in low ionic solution i.e. water and were of appreciable size for imaging. On application of $30 \mathrm{~V}$ the cells agglomerated and the biasing tips within seconds (15 to $30 \mathrm{~s}$ ). To test for selectivity the yeast cells were rendered non-viable by violet crystal staining at $60^{\circ} \mathrm{C}$ to $70^{\circ} \mathrm{C}$. These dead cells were placed in medium with live cells and biased, with only the live cells being attracted to the biasing tip while the dead cells remain un-phased. $30 \mathrm{~V}$ was applied to live cells for 30 minutes without any cell death or reproductive interference observed. It was observed that, 1) high frequency electric fields moved live cells, 2) low conductivity media, and 3) non-uniform electric fields should be considered when separating live viable cells from dead cells. Carbon nanotubes since their discovery by Ijima [14] have been the target of selective DEP for integration into CMOS technology as thermal sensors. Agarwal et al [48] integrated CNT's into a CMOS-based device using a low temp DEP process and zincation pretreatment. Parylene-C was used for improving CNT contact. The CNT's were assembled as the gain contact for an inverting amplifier circuit with I-V measurements indicating accurate thermal detection with a $-0.40 \%$ within a $25^{\circ} \mathrm{C}$ to $105^{\circ} \mathrm{C}$ 
temperature range. Similarly, Chen et al [52], assembled single wall nanotubes on CMOS for thermal sensing and observed identical thermal readings and amplifier gain measurements.

In the realm of gas sensing assembled a CNT's have found use[[49, 53], with assembly model being extended to nanowires[54, 55]. MacNaughton et al[49] assemble CNT's and grapheme based materials on Si for gas sensing, using DEP. CNT's and grapheme materials were assembled on $\mathrm{Si}$ at frequencies ranging $50 \mathrm{KHz}$ to $500 \mathrm{MHz}$, with an applied bias of 2.5 to 10 volts peak to peak. Assembly was complete within 5 minutes. Assemblies of CNT's were recorded to be $100 \%$ on every attempt. Resistance change via gas exposure yielded fractional changes in resistance in the range of $15 \%$ for vapor tested (Sarin and Soman nerve agents). Dan et al [54], assemble polymer nanowires for methanol and nitrogen gas sensing. The nanowires were synthesized using an anodic aluminum oxide (AAO) $[56,57]$. Response and recovery were rapid with device experience $90 \%$ of their change in 30 secs . The nanowires were not sensitive but not selective, expected for this system. However, nanowires did not show significant baseline drift in comparison to thin film sensors of similar properties.

\section{3) Direct Growth Integration}

The direct growth technique integrates nanostructures directly into testing devices, usually without physical handling of substrates or material pre nanowire synthesis. This integration is usually accomplished by the placement of nanowire catalyst or growth material directly on substrate via deposition (CVD, sputtering, electron beam, etc) is a technique uses bottom up synthesized nanostructures, and places them directly into sensing devices, by way

annealing and heat treatment. This technique had yielded aligned nanostructures in 
combination with the VLS method direct growth integration has been used to integrate nanowires in lateral growth structures for gas sensing using a device structure.

Using a top-down approach, Conley et al. have directly assembled $\mathrm{ZnO}$ nanowires into a working device fabricated on silicon-on- insulator substrates. [58] Using an Atomic Layer deposited conformal $\mathrm{ZnO}$ (diethyl zinc precursor and $\mathrm{H} 2 \mathrm{O}$ water vapor at $170^{\circ} \mathrm{C}$ ) seed layer, wires were fabricated without any metal catalysts. Growth occurred when substrate were exposed to $\mathrm{Zn}$ vapor at $800-915{ }^{\circ} \mathrm{C}$ for approximately $30 \mathrm{mins}$ with an Ar carrier gas flow rate of 30-80 SCCM with trace amounts of oxygen present. The fabricated bridges grew across trenches and self-terminated upon reaching a wall or flat surface. The wires were demonstrated to be functional as 3-terminal transistors and ambient gas sensors. UV (photoluminescence) characterization was performed, but yielded typical response for $\mathrm{ZnO}$. However, the transistor data showed the $\mathrm{ZnO}$ nanobridges to have poor contact resistance; this was due to non metallization pre and post processing. Authors suggested contact resistance improvement by metallization and using a lower temperature growth method that would allow for such metallization to occur without disturbing other process flow parameters. The gas sensor enabled wires were sensitive to ambient at room temperature. It was also noted that oxygen was a contributor to the conduction of the wires, providing charge species to contribute to device operation.

In Contrast, a bridging technique (bottom-up approach) to connect metal catalyzed nanowires between etch oxide grooves in Si has been developed [59-61]. Islam et al. [60] Laterally grown Si nanowires were used a connectors to bridged etch $<111>$ planes that had been previously formed by anistropically etching $<110>$ oriented silicon wafers (figure 4). These wires were made electrically conduction by doping during growth using p-type 
Borane, which was added during the chemical vapor deposition process used to synthesize the nanowires. Data from this suggests linearity and that proper contact between the electrodes wall was made. Contact resistance was not affected in the doping range tested. The average resistance across the nanowires was $350 \mathrm{k} \Omega$. The nanowires intersected the opposing wall of the etched trenches at $90^{\circ} \pm 0.5^{\circ}$. The nanowires were approximately $300 \mathrm{~nm}$ in diameter; this is estimated by the micrographs provided and knowledge of the growth conditions. Theoretical calculations and simulations revealed that nanowires with diameters of $10 \mathrm{~nm}$ would collapse under their own weight if growth distances exceeded $\sim 40 \mu \mathrm{m}$, while a nanowire of $100 \mathrm{~nm}$ could extend up $800 \mu \mathrm{m}$ before failure. These calculations neglect the influences of native oxide and residual stress induced by the growth process. Also, when nanowires terminate upon the opposite trench wall it was observed that the catalyst was present and the attachment is proposed to be of Si-Si bonding.

(a)

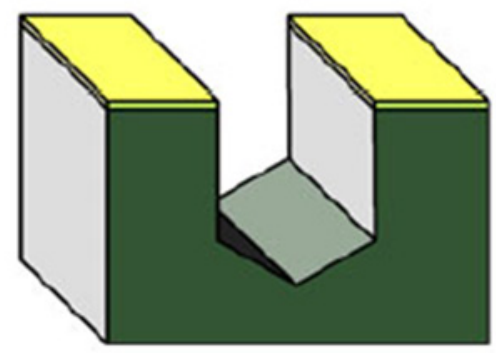

(c)

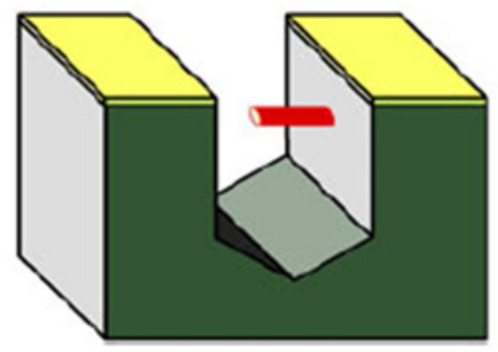

(b)

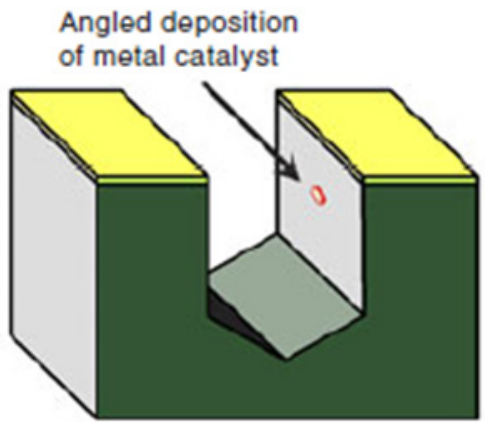

(d)

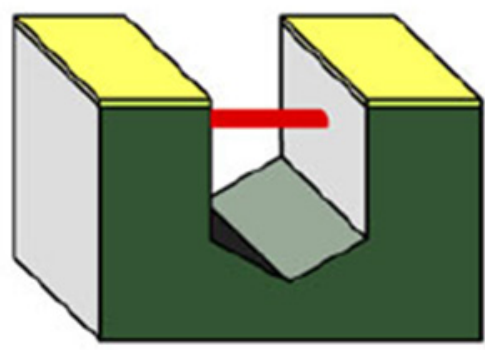

Figure 4. Schematic diagrams of (a) trench formation, (b) catalyst deposition, (c) lateral nanowire growth, and (d) bridging across trench. [Courtesy Islam [51]] 
Hochbaum et al[62] have integrated silicon based nanowires into predefined structures using with gold colloids as a seeding layer. Using a $\mathrm{SiCl}_{4}$ precursor, the VLS process was used to grow wires that were aligned and single crystalline. Process temperatures were $800-850^{\circ} \mathrm{C}$, with argon containing $\mathrm{H}_{2} 10 \%$ used as a carrier gas. The growth substrate was $<111>$ and the wires grew normal to this surface. Colloids seeds with diameters of 50 (56 \pm 5.0$), 30(30 \pm 3.3) 20(20 \pm 2.1)$ yielded wires of $93 \pm 43 \pm 4.4,39 \pm 3.7 \mathrm{~nm}$, respectively.

Traditionally, wire grown via VLS have diameters relating closely to their seed diameters, however wire diameters were slightly larger than seed colloids. It was suggested that this was due to an influx of Si into the colloids and alloy causing the seed droplets to swell in size until the super-saturation needed for wire growth occurred. Using a variation of the SNAP process the Au colloids were transferred into patterned trenches (micro channels), vertical growth was observed with the above characteristics of growth still valid.

Direct growth integration is effective due to the removal of physical handling of the nanowires and preferential placement of nanowires into preformed structures. Additionally, in the articles reviewed, the thermal budge has not been compromised during integration. However, the use of $\mathrm{SiCl} 4$ precursor poses a risk. It is hazardous and safety protocols have to be put in place before its use. It is possible that this chemical could make CMOS integration difficult, if it byproducts were to etch away trenches and gate oxide present in the existing structures. This process could be optimized if a non volatile source or precursor could be found, that would eliminate the use of hazardous materials for nanowire integration.

\section{Conclusion}

Nanostructures, such as nanotubes, nanowires, nanorods, have aided researchers in developing devices in the fields of biological and chemical sensing, energy harvesting, point- 
of-care therapeutics and more. As reviewed above nanowires have generated interest and are poised to be the next step in smaller systems. In laboratories nanowires have proven useful in single testing applications, but for commercial use a technique of mass production and system integration needs to be investigated. Therefore, the ability to synthesize them on distant and desired locations, for the purpose of performing variety of processes e.g. sensing, is of importance. A variety of concerns remain when considering integrating systems and materials, such as process parameters, system functionality, and limitations of integrated structures. To address the issue of nanowire integration into microscale systems experimentation pertaining to nanowire synthesis, biocompatibility, and integration are presented in the following chapters.

\section{Chapter 3}

\section{Experimental Methods and Fabrication Techniques}

This Chapter gives details the various experimental techniques used to explore the challenges of integrating oxide nanostructures into microscale devices. The processes presented cover the areas of metrology, thin film deposition, lithography and wet chemistry techniques.

\section{X-ray Diffraction}

X-ray diffraction (XRD) is a technique that is used for phase identification and elemental composition of materials. XRD's measure the average spacing between layers or rows of atoms to determine characteristics such as orientation, grain size, crystal structure. Additionally, measures of size, shape, and crystalline stress can also be determined. Measurements are taken when x-ray photons collide with electrons, a portion of incident beam photons will be deflected (diffracted). These diffracted waves, which are associated 
with different atoms from target surface, interfere with each other and the resultant intensity is affected by such electron interaction.

X-ray diffraction analysis works on the basis of Braggs' Law. Braggs' diffraction occurs when incident waves are diffracted from crystals and the resultant distributions are sharp interference by peaks with similar symmetry. These peaks and symmetry, if constructive can be if in multiples of $2 \pi$ can be expressed by Braggs' Law,

$$
n \lambda=2 d \sin \theta \ldots . . . \text { figure } 1
$$

Where,

$\mathrm{n}$ - An integer by the order,

$\lambda$ - Wavelength of the X-rays,

$\mathrm{d}$ - Spacing between the atomic lattice, and

$\theta$ - Angle between the incident ray and the scattering planes 


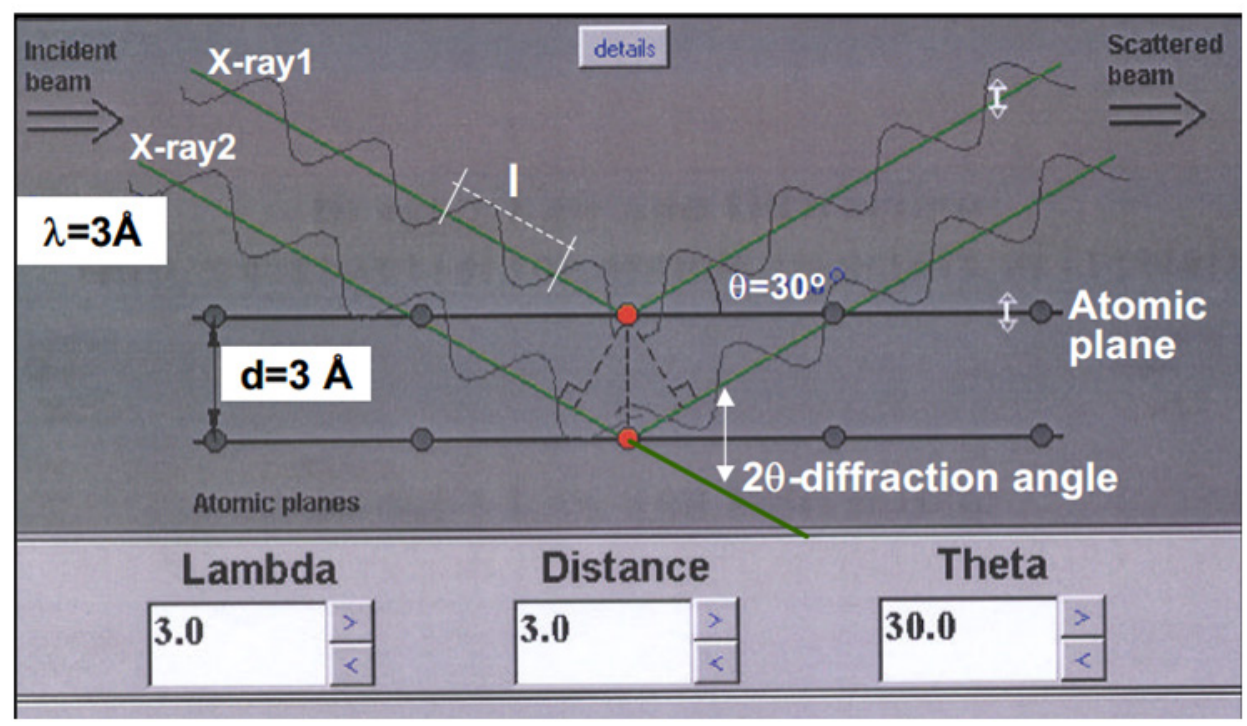

Figure 1. Simulation schematic showing fundamentals of Braggs' Law [Courtesy Stonybrook University]

XRD data presented in this dissertation was acquired using a Panalytical X'PERT PRO MRD. This device is research diffraction system equipped with hybrid and germanium (440) mono chromators, an X-ray mirror, fixed divergence slit and cross slit. Also included are triple axis \& rocking curve attachments for precision beam optic measurements. The sealed $\mathrm{CuKa}$ detectors included with this model have an efficiency of $84 \%$, with a goniometer that has a programmable step size of 0.0001 . Software and data collection was performed using Philips X'Pert Data collector, High Score for data interpretation, and Smooth fit for automated fitting, and Expitaxy (Curve fit \& simulation software) [63].

\section{Scanning Electron Microscope}

A scanning electron microscope (SEM) is a magnification device that images objects via focused beam electron rastering. Data collected from the electron object interface can be used to learned information about the morphology, crystal orientation and structure, and chemical composition of sample under study [64]. Briefly, SEM's work by producing 
magnified images using electrons, a direct contrast to traditional microscope, which use light for imaging. An electron beam (incident beam) is projected from an electron gun in vacuum. This incident beam travels vertically through electromagnetic fields and lenses which are adjusted to condition (sharpen/focus) beam towards the sample observation. The beam strikes the target and detectors collect secondary electrons, backscattered electron and X-rays to form an image that can be examined and stored (figure 2). The Hitachi S-800 was used to acquire images used in this dissertation. The Hitachi S-800 uses a cold cathode field emission source to generate electrons and is capable of 300,000x magnification of images of sample with $2 \mathrm{~nm}$ resolution. Additionally, this model is can perform Energy Dispersive Spectroscopy (EDS). EDS is a tool that can be used to perform chemical analysis on samples below 1um using an X-rays. The X-rays detected using this process are representative of the quantities of each element present in sample[65]. 


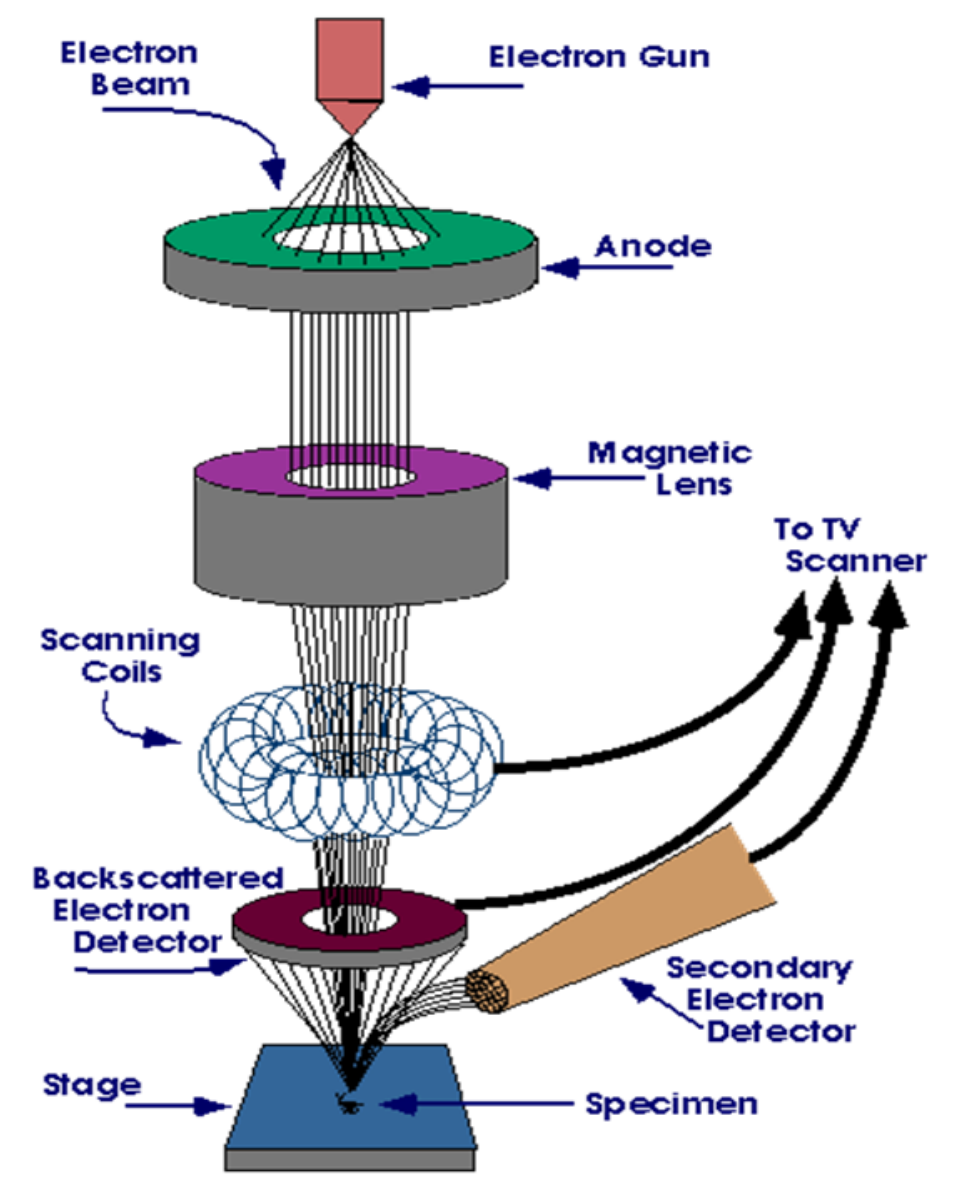

Figure 2. Scanning Electron Microscope diagram. [Courtesy Iowa State University]

\section{Transmission Electron Microscope}

Transmission electron microscopy is an imaging technique that is used to view and analyze materials at the atomic/molecular scale. Specimens examine using this technique are thin and observed on small grid structures. TEM analysis occurs when electron source emits electrons that travel through vacuum vertically. The electron beams is focus by electromagnets into a thin beam and then the beam travels into the sample, where electrons (unscattered) are collected by a fluorescent screen and analyzed through a viewer (figure 3). Objects of varying are noticeable based on their contrast in color. Transmitted (unscattered) electrons form bright field images. In contrast, diffracted (scattered) electrons form dark field 
images. Conventional TEM studies of crystalline materials often use the diffraction contrast mechanism (which is the variation of intensity of electron diffraction across the sample) to produce either of the images. Bright field and dark field images are complementary, usually displaying contrasting views of images, for the purpose of revealing shaded/ in-depth morphological features.

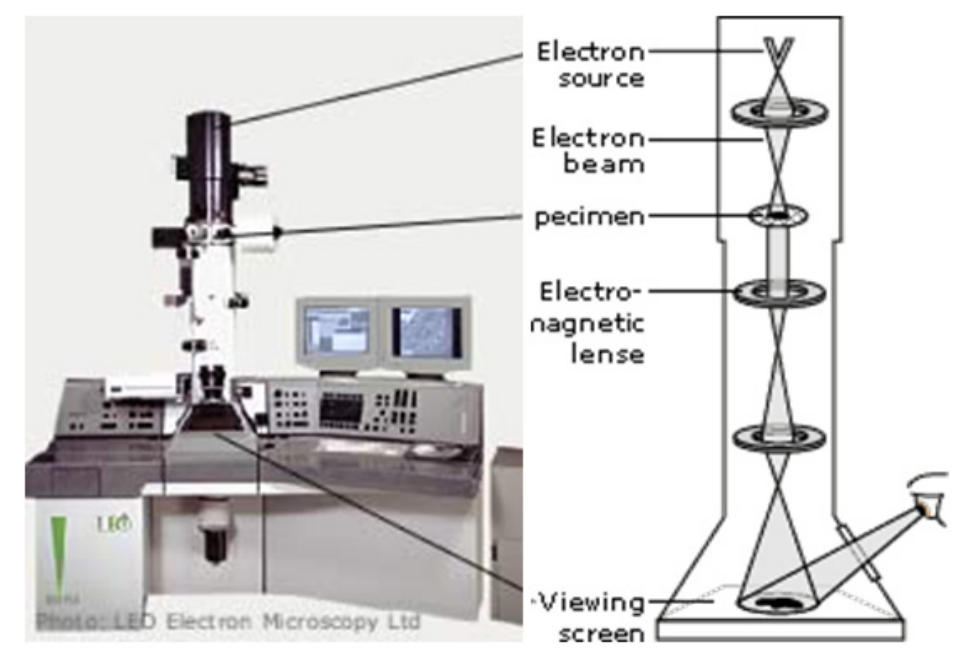

Figure 3. Transmission electron microscope and diagram. [Courtesy Nobel Peace Prize.org]

TEM data presented in this dissertation was acquired using a Tecnai F20. The Tecnai F20 is high resolution device outfitted with both bright and dark field detectors for high resolution imaging. Additionally, the microscope uses a Schottky field emitter as an electron source, high beam stability and long life. The device also features a point and line point resolutions of $.24 \mathrm{~nm}$ and $.102 \mathrm{~nm}$, respectively, with a camera length $30 \mathrm{~mm}-4.5 \mathrm{~m}$ and an accompanying diffraction angle of $26^{\circ}$ and double tilt angle for the specimen of $40^{\circ}$ [66].

\section{Experimental Methods in current research}

4 inch n-type silicon wafers were coated with metal catalyst via thermal evaporation. 
Initially, $\mathrm{Si}<100>$ wafers were used with $\mathrm{Au}$ and $\mathrm{Pd}$ catalyst for initial weight gain and growth studies. However, for vertical alignment experiments $\mathrm{Si}<111>$ substrates with $\mathrm{Au}$ and Pd catalyst were used for nanowire synthesis. The metal deposited wafers were cleaned in buffered oxide etchant, to remove native oxide and hydrogen peroxide, to remove organics and dried with nitrogen. Then the substrates were diced into $1 \mathrm{~cm} \times 1 \mathrm{~cm}$ pieces, for placement into furnace for high temperature annealing.

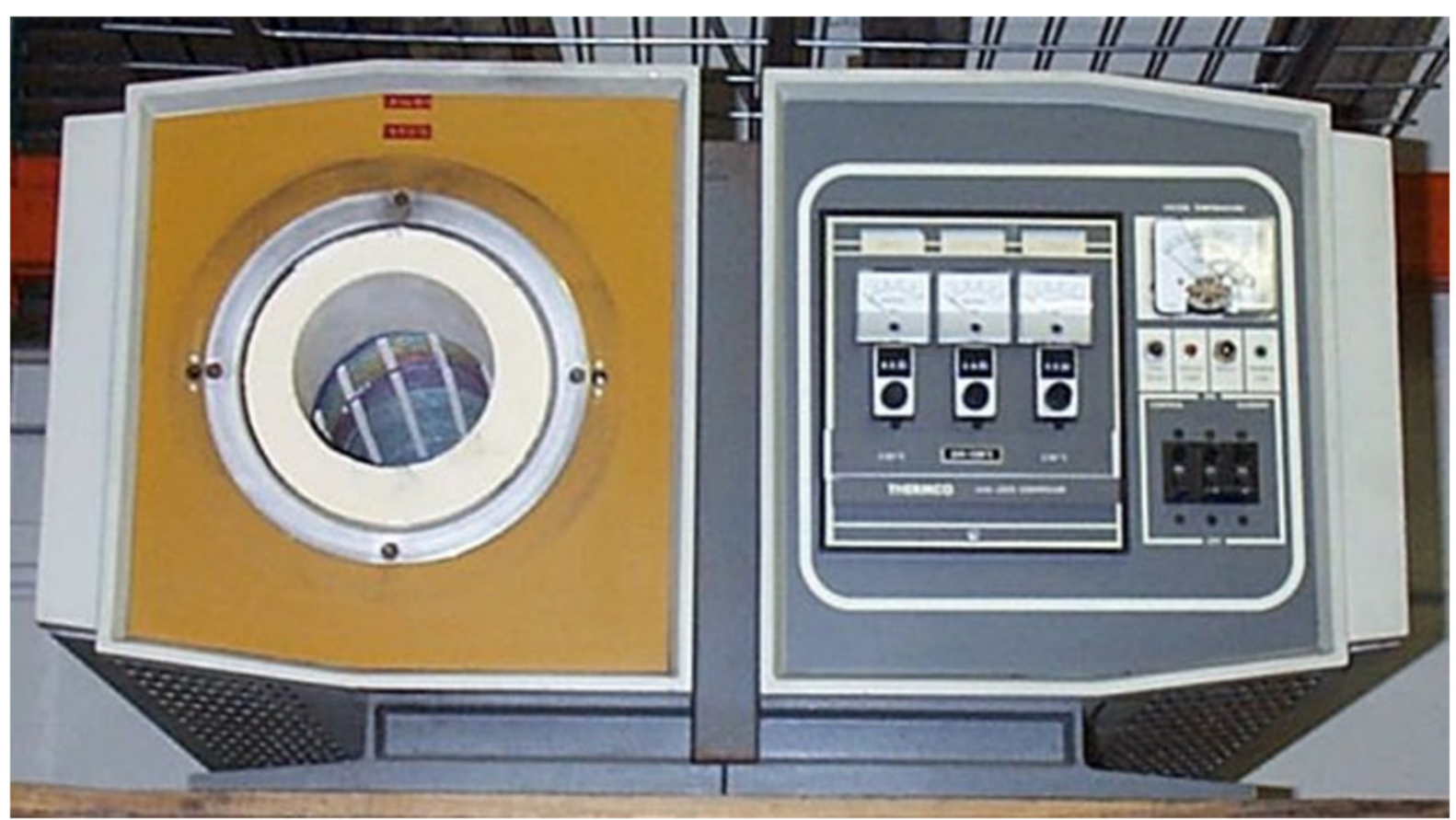

Figure 4. Thermco MB-71 Laboratory Diffusion Furnace [Courtesy Surplus Process Equipment Corp]

An open tube furnace(figure 4), the Thermco MB-71, was used to induce nanowire growth via the Vapor Liquid Solid growth mechanism[8]. The furnace is a three zone unit featuring; water cooled heating elements, operating temperatures between $200^{\circ} \mathrm{C}$ to $1200^{\circ} \mathrm{C}$, and with a tube diameter of 4.5 inches and length of 30 inches. Nanowire growth trials were performed at $1050^{\circ} \mathrm{C}$, for a range of times varying from $5 \mathrm{~min}$ to 240 , using the parameters listed in figure 5. The high temperature furnace was ramped up to $1050^{\circ} \mathrm{C}$ at $17.5^{\circ} \mathrm{C}$ per 
minute. During ramp-up Argon was used as a purging and carrier gas and (25 SCCM flow rate) was to remove contaminating species present in the furnace tube. Pd sputtered samples were placed on a small silicon wafer (donor substrate) and introduced into the furnace and heated.

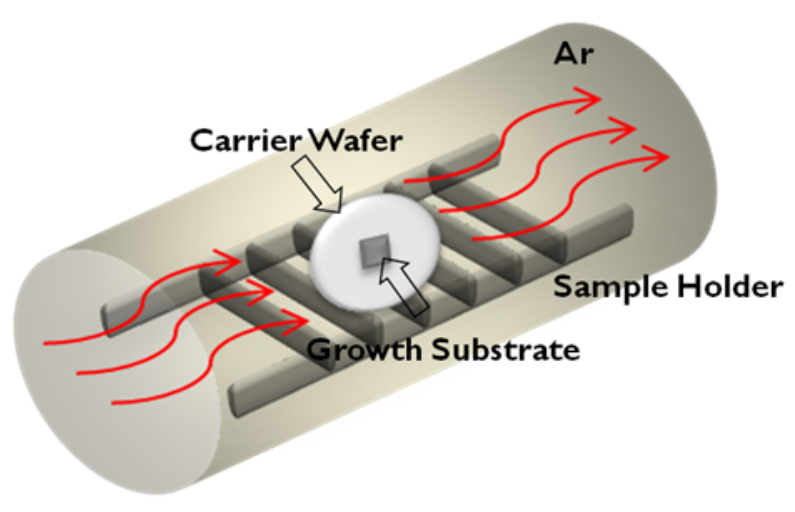

\begin{tabular}{ll}
\hline \multicolumn{2}{c}{ Growth Parameters } \\
\hline Growth Substrate & Silicon $<100>$ \\
\hline Catalyst & $\mathrm{Pd}$ \\
\hline $\begin{array}{l}\text { Soak (Growth) } \\
\text { Time }\end{array}$ & $5-120$ Minutes \\
$\begin{array}{l}\text { Growth } \\
\text { Temperature }\end{array}$ & $1050^{\circ} \mathrm{C}$ \\
$\begin{array}{l}\text { Growth Mechanism } \\
\text { Vapor-Liquid-Solid } \\
\text { (VLS) }\end{array}$ \\
\hline Gas Flow & 25 S.C.C.M. \\
\hline
\end{tabular}

Figure 5. Quartz-tube furnace setup and growth parameters used for nanowire synthesis.

The prepared samples were weighed pre and post heating, to determine the substrate weight change, using a high precision microbalance. After calibration with a known calibration sample, the nanowires samples were placed on the scale and sealed within its plexiglass walls and then measured. Results from this study are presented as an appendix in this dissertation.

Nanowire integration into interdigitated microelectrode structure was performed at $1050^{\circ} \mathrm{C}$, with a growth (soak time) of $60 \mathrm{mins}$ on oxidized $\mathrm{Si}<111>$ substrates. Morphological characterization of the wires was carried out using Hitachi SEM S800, S4800 
SEM) fitted with an EDS detector. High resolution structural studies were carried out using transmission electron microscopy (FEI Tecnai F30 TEM) with SAED and EDS capabilities. Surface functionalization of these nanowires was done using Prostate antibody solution. For modification, a PSA antibody solution $(10 \mathrm{mM}, \mathrm{pH} 7.0,0.9 \% \mathrm{NaCl})$ containing $5 \mathrm{mM}$ $\mathrm{Fe}(\mathrm{CN}) 63-/ 4-$ was placed on the devices and allowed to incubate for $24 \mathrm{~h}$. The sensor was then washed thoroughly with PBS $(10 \mathrm{mM}, \mathrm{pH} 7.4)$, In this case the phosphate buffered solution was used to remove any unbound biomolecules on the electrode surface and then incubated with a solution containing prostate specific antigens.

\section{Chapter 4}

\section{Growth Characteristics of Metal Catalyzed Silica Nanowires}

I. Introduction

Synthesizing nanowires is of great importance in understanding their properties and preparing them for sensing applications. Future sensors will require a refined growth technique that will yield wires that can be modified for increased sensitivity. The VaporLiquid-Solid (VLS) growth technique has been used successfully in the synthesis of the metal catalyzed nanowires. During VLS growth, a metal droplet acts as the sight for a vapor phase Si absorption. From the supersaturated droplet, Si precipitation occurs at the droplet site and Silica nanowires grow simultaneously consuming/dispersing the metal catalyst along the length of the wire and into growth chamber.

Metal catalyzed 1-D nanostructures, such as nanowires, have the potential to revolutionize technological areas such as personal health care and homeland security $[67$, 68]. This is due to their adaptable morphology, which has led to nanowire use in 
environmental monitoring and cancer therapeutics $[69,70]$. The next step in nanowire evolution is their integration into devices usable for in-vivo drug delivery and measurement of intra-muscular activity. However, one of the primary challenges of producing devices such as these is developing a method that yields nanowires of reproducible morphology and chemical composition. Developing a method for controlled and oriented growth of nanowires that lends itself to large-scale manufacturing is needed for their commercialization.

Among these, palladium (Pd) and gold ( $\mathrm{Au})$, transition metals have been used as a catalyst in VLS nanowire growth, due to occurrence of Pd-Si eutectic at $760^{\circ} \mathrm{C} .[71]$ and $\mathrm{Au}-$ Si eutectic at $360^{\circ} \mathrm{C}[29]$. We report the use of the VLS growth mechanism with $\mathrm{Au}$ and $\mathrm{Pd}$ as a growth catalyst for $\mathrm{SiOx}$ nanowire synthesis at $1050^{\circ} \mathrm{C}$ and $1150{ }^{\circ} \mathrm{C}$. These temperatures are chosen as an intermediary and outlier values, respectively, to provide a comprehensive representation of the influence of temperature and time on nanowire synthesis.

In the paragraphs below results nanowire growth factors were explored and investigated to determine simple, reliable and reproducible techniques for controlled, bottomup manufacturing of silica nanowires for integration into sensing structures for mass manufacturing. The results of the studies explain the role of temperature and time on nanowire morphology.

\section{Growth of Amorphous Silica Nanowires via VLS on $<100>$ Si}

A. Experimental 


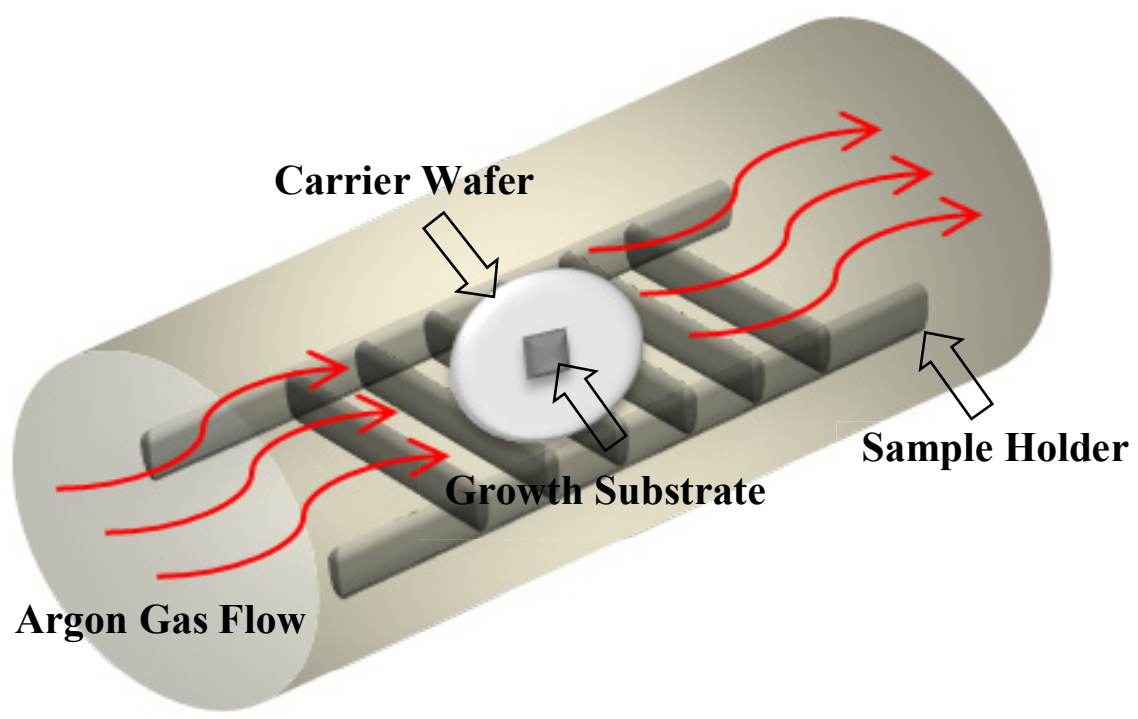

Figure 1. Diagram illustrating sample setup and gas flow into furnace tube.

Silica nanowires were grown on 4 -inch $\mathrm{Si}<100>$ wafers. Initially, the wafers were cleaned using a standard RCA cleaning procedure to remove any organic and inorganic contaminants, and then deposited with a thin layer of growth catalyst (5 nm of Pd) and diced into $1 \mathrm{~cm}^{2}$ coupons. Later, the coupons were placed into an open tube furnace for nanowire synthesis via the VLS growth mechanism (Fig 1). During the initial ramp-up of the furnace, argon (99.9\%) was used as a purging and carrier gas, to remove contaminating species from the heating tube and to aid silicon transport during growth, respectively. 


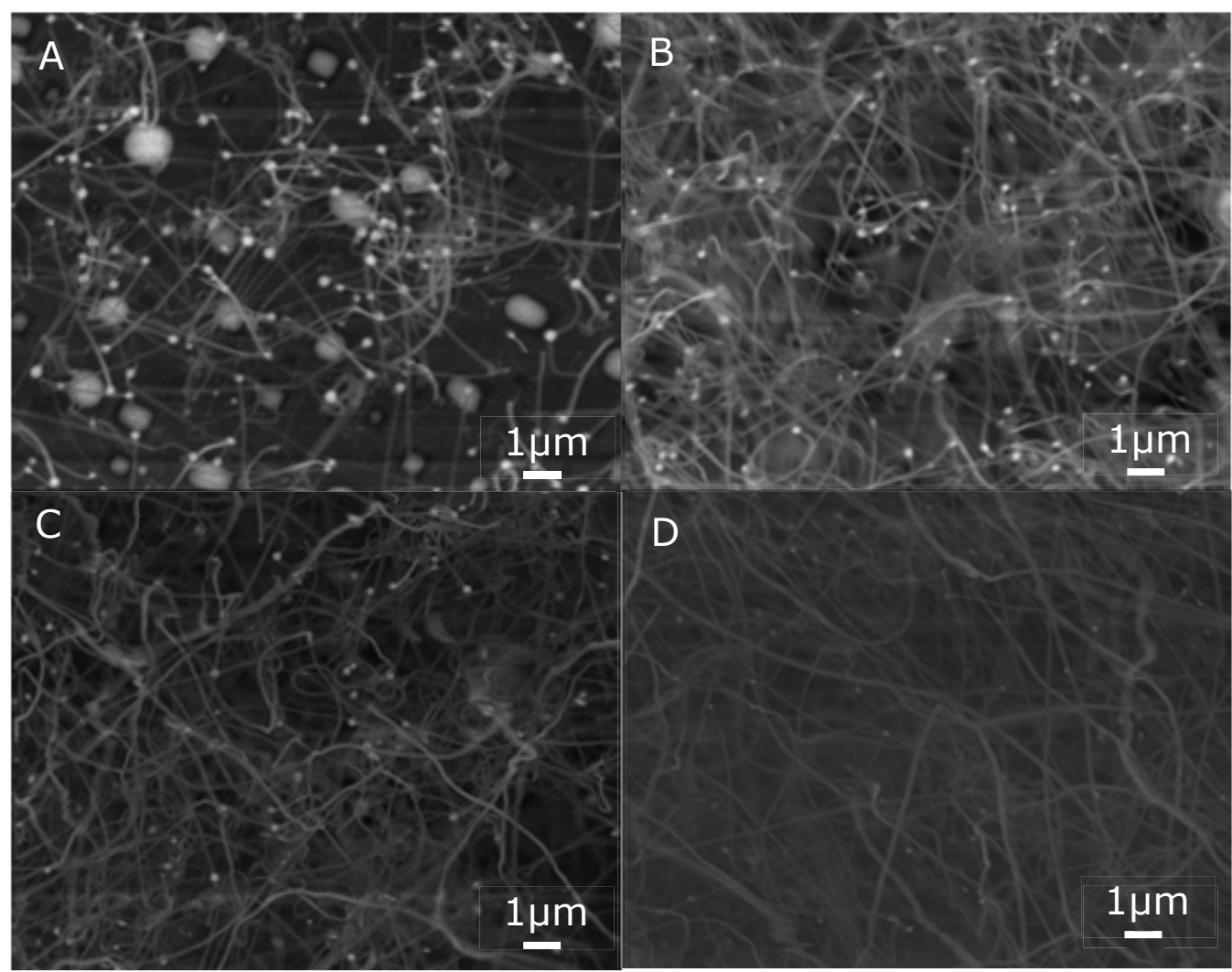

Figure 2. Time stamp at $1050^{\circ} \mathrm{C}$ showing catalyst evaporation for nanowire growth a) $5 \mathrm{mins}$, b) $20 \mathrm{mins}$, c) $75 \mathrm{mins}$, and d) $90 \mathrm{mins}$

Nanowire growth trials were performed at two temperatures, $1050^{\circ} \mathrm{C}$ and $1150^{\circ} \mathrm{C}$. The growth temperatures were chosen based upon the Pd-Si phase diagram[30] and previous results. Samples were heated for various times $(5,10,15,20,30,45,60,75,120$, and 240 mins) so that catalyst development and growth morphology could be monitored and growth conditions recorded.

Scanning electron microscopy (SEM), energy dispersive x-ray spectroscopy (EDS), and transmission electron microscopy (TEM) were used to monitor catalyst evaporation, verify elemental composition, and determine nanowire morphology, respectively. 


\section{B. Results and Discussion}

Figure 2a-d is a SEM composite showing Pd catalyst consumption at $1050^{\circ} \mathrm{C}$ for 5 , 20,75 , and 90 min intervals. Nanowire growth was immediately noticeable at 5 minutes of annealing. As the annealing time was increased the wires grew longer and began to appear denser covering the substrate by the 20 minute time mark.

Between 20 to 75 minutes, an accelerated nanowire growth was observed (not shown). Also the metallic catalyst located at the tips of the nanowires was noticeably smaller. Beyond 75 minutes, growth in z-axis was observed to be significantly slowed. At 90 minute mark the metal catalyst was nearly evaporated. Figure 3 depicts Si etch pits with Pd globules sunken wafer surface (nanowires removed).

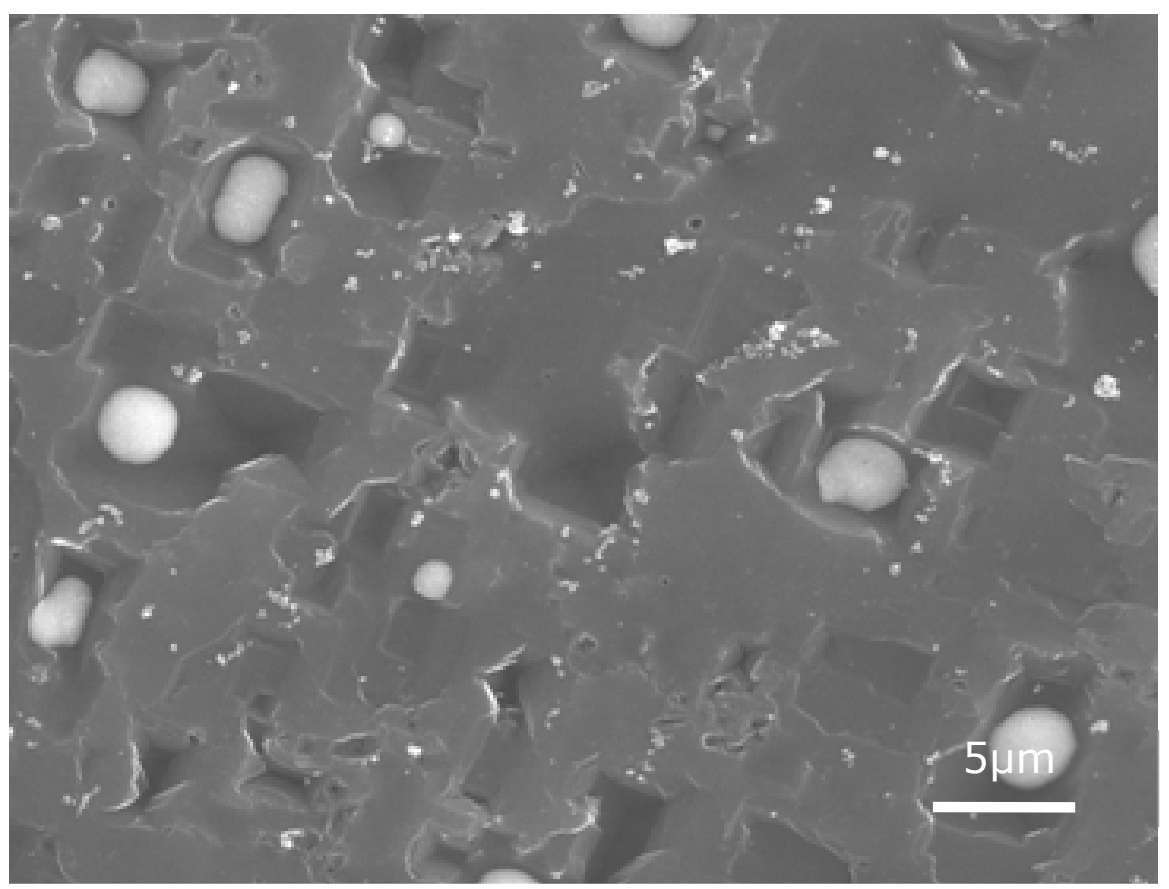

Figure 3. Silicon etch pits on surface of wafer, with nanowires removed. 


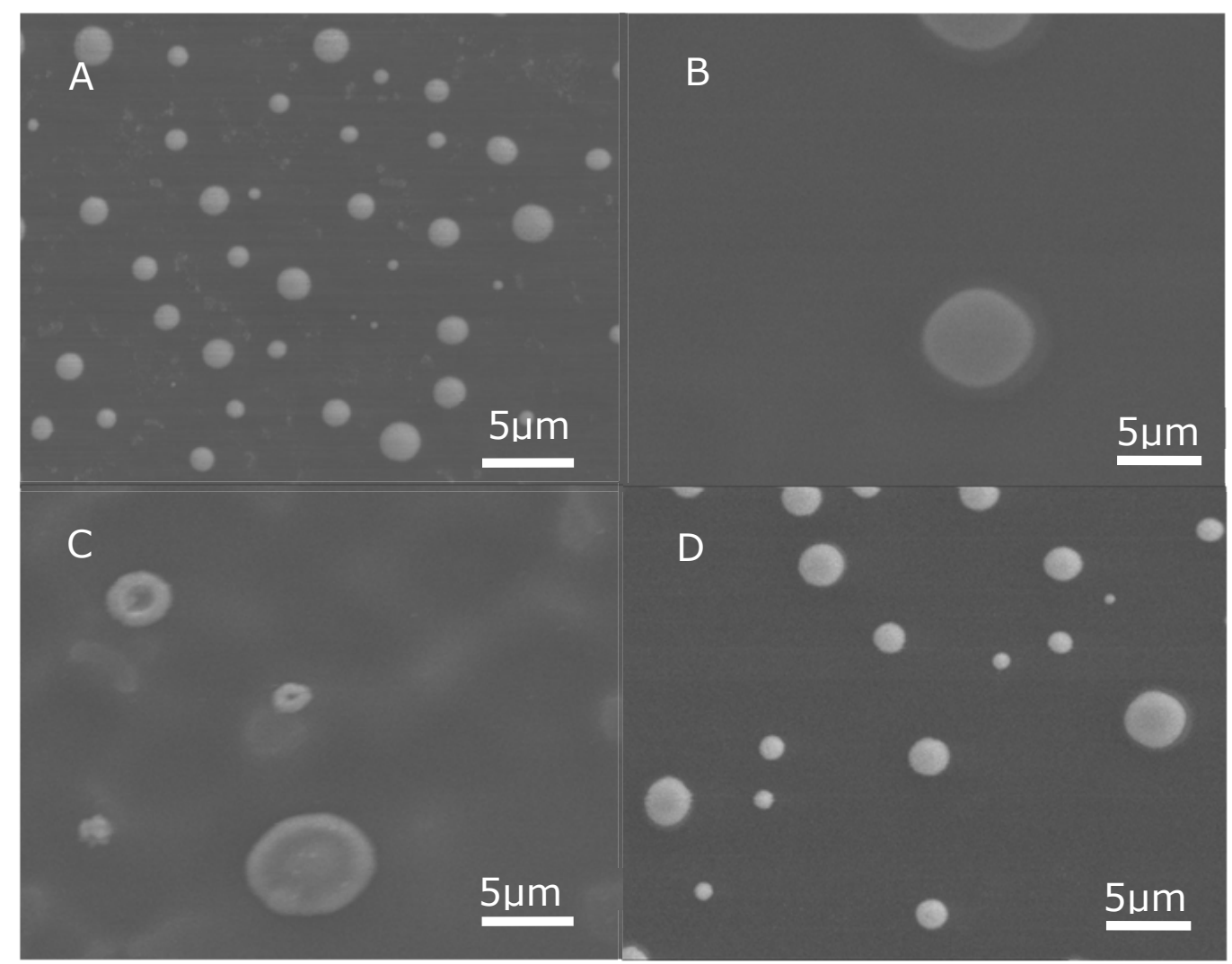

Figure 4. SEM images showing stunted nanowire growth at $1150^{\circ} \mathrm{C}$ for a) $5 \mathrm{~min}$, b) $20 \mathrm{~min}$, c) $90 \mathrm{~min}$, and d) $120 \mathrm{~min}$ time stamps.

Next, Pd deposited Si substrates were subjected to $1150^{\circ} \mathrm{C}$ using the same processing andfabrication steps used for the $1050^{\circ} \mathrm{C}$, to establish a nanowire growth boundary. However, growth trials did not yield any nanowires. Figure 4, an SEM composite, confirmed the presence of scattered Pd globules, but absent of nanowires. Further, the expected substrate trenches from silicon etching were also not present.

Typically, the nanowires continue to grow till the catalyst $(\mathrm{Pd})$ is unavailable. This trend can be observed at $1050^{\circ} \mathrm{C}$ as shown in Figure 2a-d. When the growth condition was extended beyond 90 mins, after the catalyst was consumed, growth was observed lengthwise. 
However, lateral growth was observed at higher time scale. This lateral growth is typically attributed to a combination of VLS and OAG mechanism as suggested by Sekhar [17]. OAG is characterized as nanowire growth without the presence of metallic catalyst at high temperatures $[27,72]$. However, nanowires synthesized at $1050^{\circ} \mathrm{C}$ exhibited the presence of $\mathrm{Si}$ and $\mathrm{Pd}$, suggesting that during growth the catalyst is dispersed in the growth chamber and along the stem of the nanowires. Figure 5 shows the Transmission Electron Microscopy image and Energy Dispersive x-ray Spectroscopy data of the same wires, verifying the catalyst presence in the nanowires. Since the temperature of the furnace was kept constant during growth for all annealing times, it is possible that the presence of the dispersed $\mathrm{Pd}$ particles along with vapor phase $\mathrm{SiO}$ contributed to the lateral development of the wires.
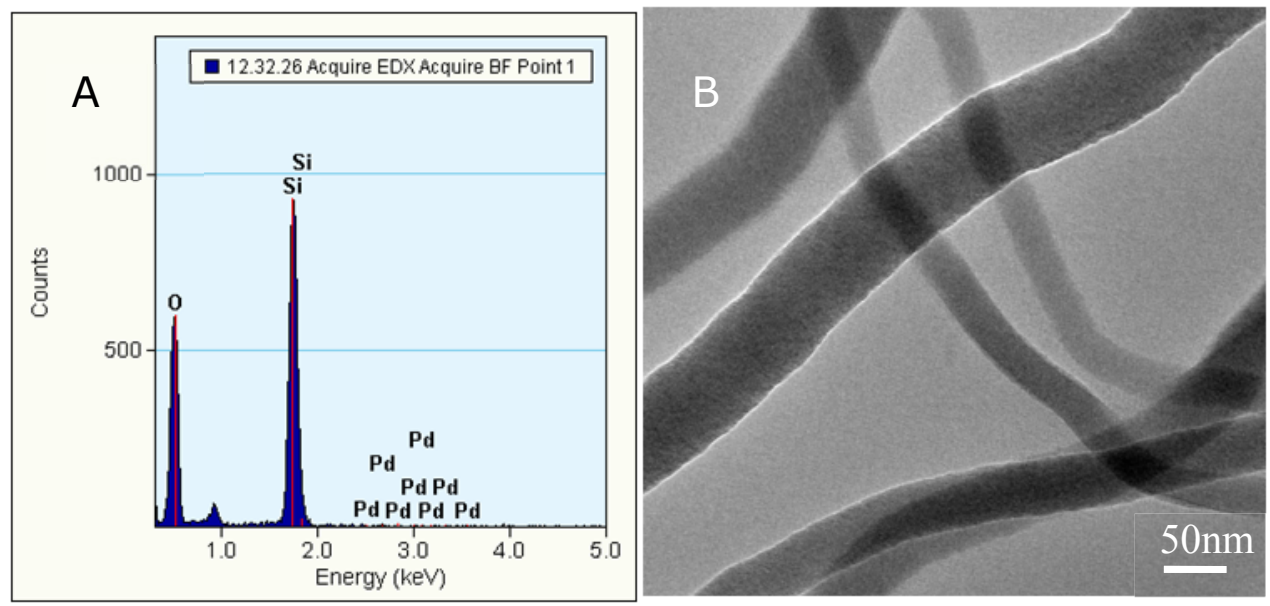

Figure 5. (A) EDS on silica nanowire sample revealing the presence metallic catalyst $(\mathrm{Pd})$ dispersed along the length of the wire. (B) Transmission Electron Micrograph (TEM) of silica nanowire without palladium visible.

Furthermore, during the initial growth stages of silica nanowires at $1050^{\circ} \mathrm{C}$, etch pits were observed as shown in Figure 2a and shown again in Figure 3(without nanowires). This was caused by the uneven dissolution/ evaporation of silicon from the substrate. When annealed in high temperature atmospheres in the presence of low oxygen partial pressure, 
surface roughening of silicon substrate occurs, causing silicon to be etched from the surface instead of being oxidized, as identified by Suzuki [73] and observed by Sekhar [74], Yang[75], and Kim[76] in their reports on oxide nanowire manufacturing, growth environment characterization and nanowire functionalization, respectively. However, this $\mathrm{Si}$ surface removal and subsequent pit formation serves as the main source of silicon for nanowire formation. At $1050^{\circ} \mathrm{C}$, VLS has been the dominant growth mechanism. A simplified growth model depicting four distinct stages i.e., a) oxide formation, b) nanowire nucleation and $\mathrm{Si}$ etch pit formation, $\mathbf{c}$ ) nanowire growth, and d) nanowire growth saturation is shown in Figure 6.

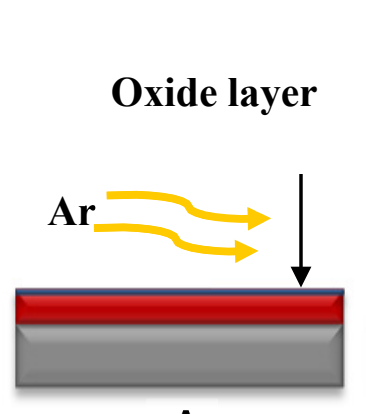

A

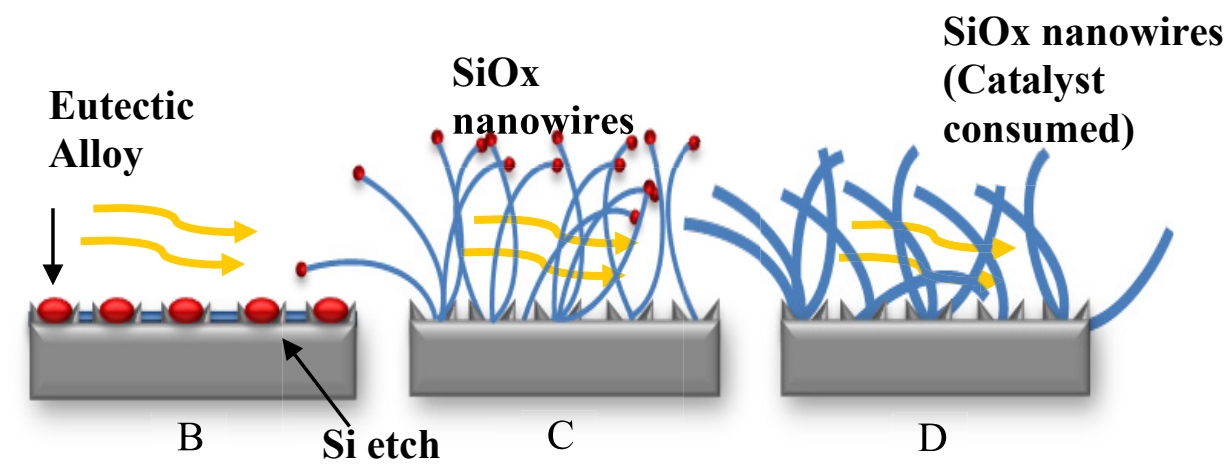

Figure 6. Schematic Illustration of metal catalyzed VLS growth at $1050{ }^{\circ} \mathrm{C}$,

A) Deposition of Catalyst, B) Eutectic Liquid Alloy and Si pit formation,

C) Nanowire Growth, and D) Saturated nanowire growth

The growth of $\mathrm{Pd}$ catalyzed silica nanowires at $1100^{\circ} \mathrm{C}$ suggested that nanowire synthesis occurred in two separate phases, VLS and OAG [27], and that growth was reliant on $\mathrm{SiO}$ vapor for continued growth. Direct comparison of data at $1100^{\circ} \mathrm{C}$ [74] and $1050^{\circ} \mathrm{C}$ revealed that nanowires produced at the higher temperatures had thicker diameters and longer lengths than those produced at $1050^{\circ} \mathrm{C}$. A separate experiment was performed with annealing temperature set to $1150^{\circ} \mathrm{C}$ for the purpose of growing wires that would be larger and heavier compared to those grown at $1050^{\circ} \mathrm{C}$ and $1100^{\circ} \mathrm{C}$. Growth trials at the $1150^{\circ} \mathrm{C}$ yielded results 
contradictory to $1050^{\circ} \mathrm{C}$ growth patterns (Figure 4), stunted nanowire growth. We have observed that the presence of oxygen in the ambient environment and native oxide on $\mathrm{Si}$ substrate surface both serve as contributors to the growth of nanowires in an open tube furnace growth systems. Imbalance's in this gas equilibrium has been observed to be detrimental to nanowire growth [17]. However, it is the change in temperature from $1050{ }^{\circ} \mathrm{C}$ to $1150{ }^{\circ} \mathrm{C}$ that has caused the VLS process to be halted.

When the nanowire synthesis temperature is increased the $\mathrm{O}_{2}$ (provided by $\mathrm{SiO}$ from wafer dissolution) needed for growth becomes limited due to an increase in vapor pressure (Fig 7). At the $1050^{\circ} \mathrm{C}$ and lower temperatures growth was possible with the use of the additional oxygen source (ambient). However, at $1150^{\circ} \mathrm{C}$ the ambient source is not enough (well above $10^{-5}$ needed for Si surface etching) Thus, Si etching of the wafer is prevented, super saturation of Pd globules does not occur and nanowire growth is halted. This effect is suggested to explain the stunted growth observed at $1150^{\circ} \mathrm{C}$. Moreover, observations from the above experiments provide insight into techniques that can be used to integrate nanowires into Si based structures, by adjusting oxygen partial pressure in conjunction with process temperature to inhibit etch pit formation or site specific nanowire placement. Recently, the above growth conditions and observations were used to integrate silica nanowires into microelectrode structures for chemical sensing application[2]. Efforts are being made to integrate these nanowires into CMOS platforms for environmental monitoring. 


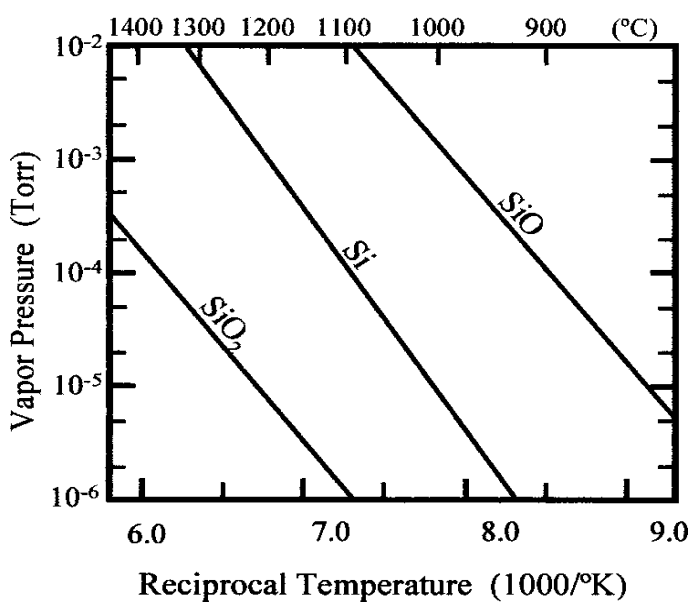

Figure 7. Equilibrium vapor pressures of silicon and its oxides. "Reprinted with permission from [Suzuki, T., Effect of annealing a silicon wafer in argon with

\section{Conclusion}

Metal catalyzed silica nanowires were grown using the VLS growth mechanism and the effect of temperature and time on growth has been reported. The composition of the nanowires was found to be primarily silicon and oxygen with Pd dispersed along the wire stem. These experiments establish that nanowire growth can be tailored through control of growth parameters. Using such controls silica nanowires were grown with diameters of $125 \mathrm{~nm} \pm 5 \%$. This has been established through the controlled study of effect of time and temperature. The wires stop growing at $1150^{\circ}$ due to lack of $\mathrm{Si}$ vapor provided by the low oxygen partial pressure etching of Si substrate.

\section{Growth Optimization of Silica nanowires catalyzed with Au on <111>}

Using current growth processes the VLS mechanism can be used to yield dense forests of nanowires and with the inclusion of metal catalysts these wires can be tailored and their morphologies i.e. diameter and length can be predictable.. However, the VLS process is a parasitic process by design. This is due to the removal of silicon from the growth substrate surface, used for wire growth. This removal of the growth substrate could prevent the 
integration of VLS grown nanowires into CMOS structures due to possible device damage during nanowire integration. Below a technique is presented that demonstrates the use of a capping layer to address this silicon dissolution from the growth substrate.

\section{A. Experimental}

Silica nanowires were grown on 4-inch $\mathrm{Si}<111>$ wafers, to determine the role of substrate orientation in wire growth. The wafers were cleaned using a standard RCA cleaning procedure to remove any organic and inorganic contaminants, and then deposited with a thin layer of growth catalyst $5 \mathrm{~nm} \mathrm{Pd}$ and $\mathrm{Au}$ and then diced into $1 \mathrm{~cm}^{2}$ coupons. The coupons were placed into an open tube furnace for nanowire synthesis in three different configuration involving a carrier substrate (to hold the wafer), growth substrate (with $5 \mathrm{~nm}$ metal film), and a carrier wafer (to provide source of Si). During the initial ramp-up of the furnace, argon (99.9\%) was used as a purging and carrier gas, to remove contaminating species from the heating tube and to aid silicon transport during growth, respectively. The growth temperature was chosen based upon the Pd-Si [30] and Au-Si diagrams and previous results. For aligned nanowire growth and $<111>$ substrate is used based on Wagner and Ellis' $[8]$ original assumptions concerning the whiskers growth via VLS Figure 8a. To prevent growth substrate etching via the VLS a Si capping layer is used as the primary source of vapor phase $\mathrm{Si}$ instead the growth substrate figure $8 \mathrm{~b}$. The capping layer is kept at a distance of $500 \mu \mathrm{m}$ away from the growth substrate surface, so as to interfere with the length of the wires as they grow from the substrate.
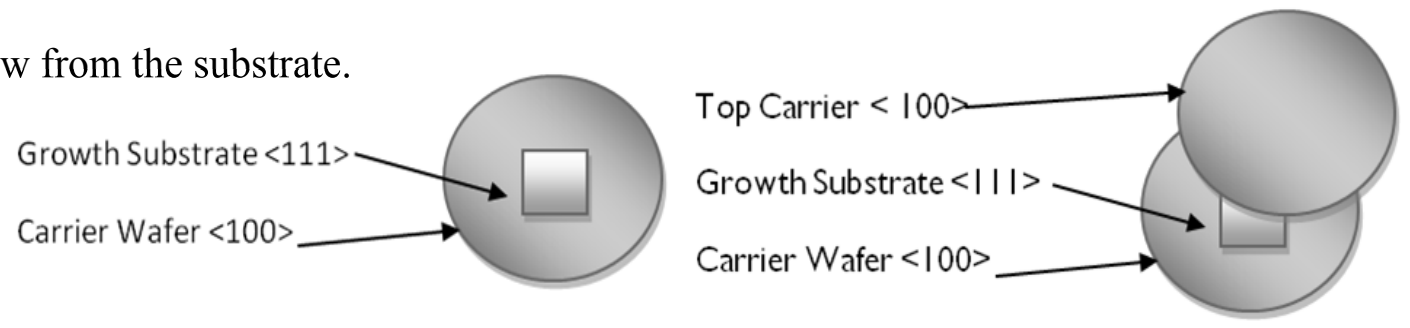

Figure 8. a) Diagram of $<111>$ Si substrate setup for aligned nanowire growth, b) diagram of $<111>\mathrm{Si}$ wafer for aligned growth with $<100>$ cap layer used to prevent growth substrate etching during nanowire svnthesis 


\section{B. Results and Discussion}

Figure 9a-c is an SEM composite of silica nanowire growth catalyzed with Au at 1050C for 60 minutes. These wires were grown using the substrate setup in figure 8a. Due to the longer growth time the substrate floor is densely covered with nanowires. From the micrographs nanowire growth was observed to be more vertically aligned and oriented when compared to samples grown at the same temperature and time duration on $<100>$ substrates. The surface of the growth substrate was smooth; however evidence of Si etching was noticeable, as indicated by groove pits beneath the wires (fig 9b, c). This is evidence that Si dissolution did occur and that the primary source of $\mathrm{Si}$ in this growth method is still from the substrate itself. Metal particles (Au globules) (Figure 9b) are still noticeable at the tips of the wires and the nanowire diameter is in of $100-150 \mathrm{~nm}$ in agreement with previous by work presented in this chapter. 


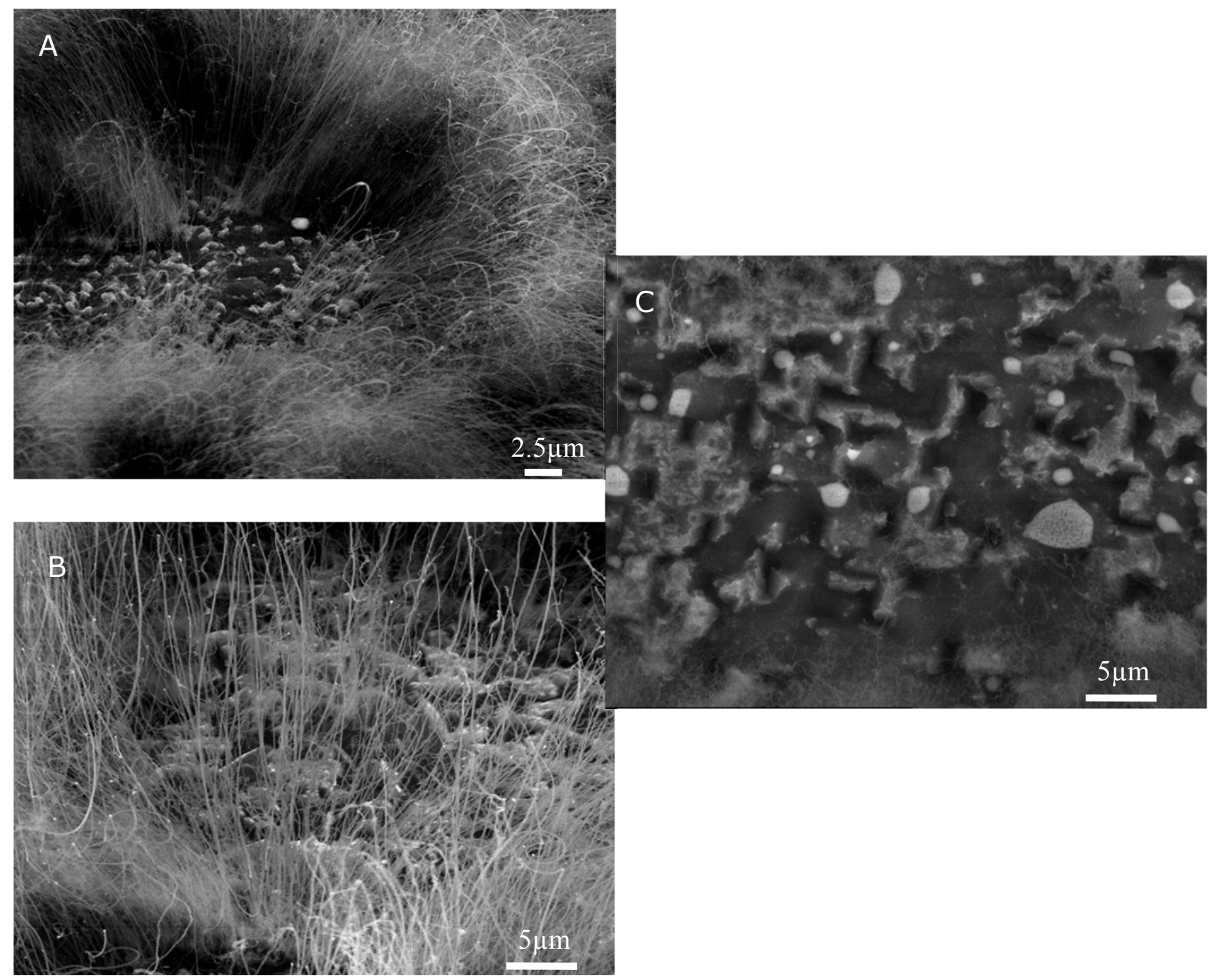

Figure 9. SEM composite of nanowire $\mathrm{Si}<111>$ growth substrate a) nanowire growth with noticeable wafer pitting /etching, b) magnification showing aligned wires with metal tips and etch pits in background, c) magnified area showing etch pits and isolation growth patches

Also, the carrier wafer had noticeable nanowire growth and etch pit formation. Au was not deposited on this substrate, so catalytic VLS nanowire growth was not expected. It is possible that nucleated droplets on the growth substrate during the vapor phase could have migrated, due to carrier gas flow (Ar) onto the carrier wafer, to cause nanowire growth. However, while etch pits were noticeable, revealing the Si wafer dissolution, the metallic tips 
that have been observed in all the previous experiments were not present, the signaling the possibility of another growth mechanism occurring concurrently during metal catalyzed VLS. Micrographs (Figure 10) of the wires show them to be randomly oriented, expected nanowire growth on a $\mathrm{Si}<100>$ surface. The diameters (Figure 10d) on the growth substrate were similar those observed of metal catalyzed wires grown on $<100>$ substrates, and edax (Figure 11) showed the area containing the wires to be of silicon and oxygen content. These observed results were interesting but were not researched any further and concluded to be related to the oxide assisted growth method (OAG) discussed in chapter 2.

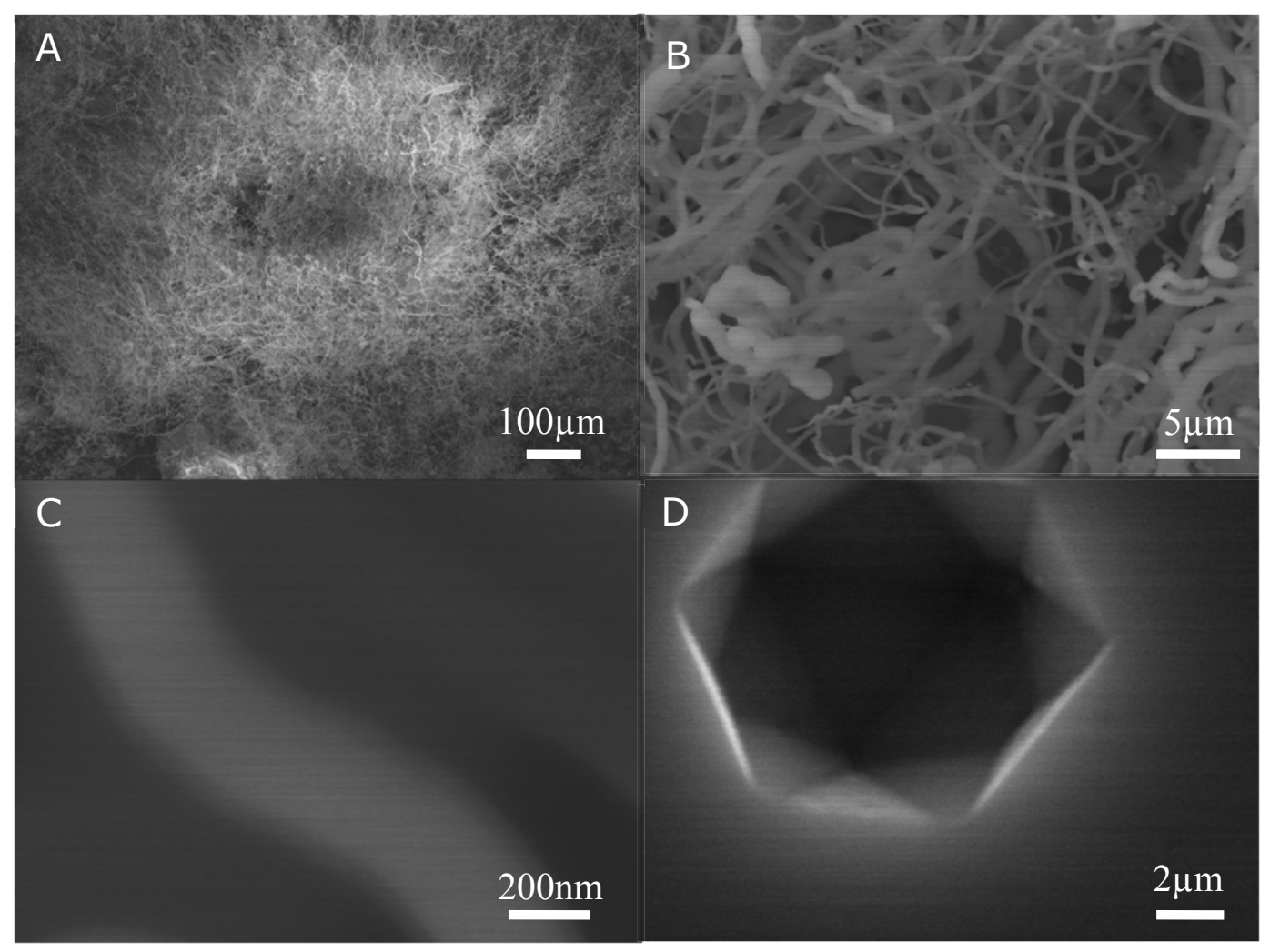

Figure 10. SEM composite of nanowire growth on carrier substrate a) Randomly oriented growth, b) Zoom in on same area, c) 70K magnification of single wire, and d) etch pit on carrier wafer surface 


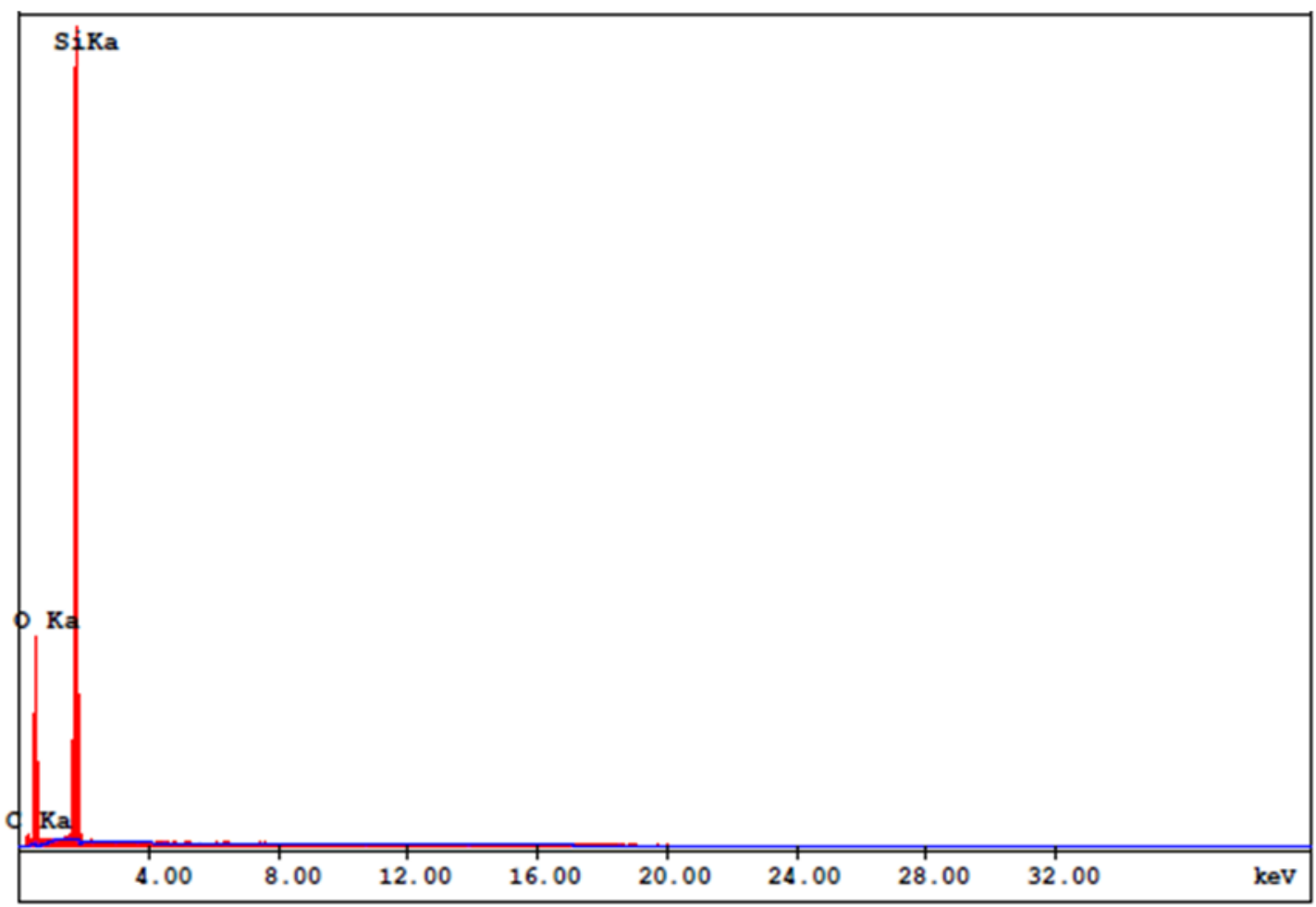

Figure 11. EDAX of area on a carrier wafer containing nanowires confirming the presence of $\mathrm{Si}$ and $\mathrm{O}$, absent metal catalyst.

Next, Figure 12a-c is a composite micrograph of the nanowires prepared using the growth setup in figure $8 \mathrm{~b}$. Overall the nanowires were observed to more vertically align than those grown on $\mathrm{Si}<100>$ wafers. The bending of the wires is attributed to the carrier gas force used to drift Si on metallic globules to influence growth. Metallic tips were observed at the end of the nanowire stems and edax of the area confirmed the presence of $\mathrm{Si}, \mathrm{O}$ and $\mathrm{Au}$, as expected (Figure 12d). The substrate surface was smooth and clear of the etch pits expected, when nanowire synthesis occurs via VLS. This is attributed to the use of the topside capping layer above the growth substrate. This capping layer changes the primary source of silicon, thus preserving the growth wafer substrate, but in Figure 12c, surface grooves/ridges are present. The contributions of the ridges are unknown, but wire growth in these areas was not present, an indication that the carrier etch formation occurred on the capping layer (figure 13). However, an alternate theory of etch pit formation on top carrier 
can be linked to the possible evaporation of Pd catalyst onto top carrier surface. This is possible due to the micron separation length of the growth substrate and top carrier. For the catalyst to evaporate and redeposit on the top carrier and continue the VLS process, the catalyst would need to boil off the surface of the growth substrate. The enthalpy of vaporation for $\mathrm{Pd}$ is $357 \mathrm{~kJ} / \mathrm{mol}$ measured at its boiling point of $2963^{\circ} \mathrm{C}$. These values are not in agreement with VLS growth environment used to synthesize silica nanowires. However, the eutectic system of Si-Pd introduces a new energy system not addressed by traditional enthalpy values and measures. Initially, the etch pits on the top carrier wafer are attributed to the dissolution of Silicon which has been reported to occur at the processing parameters of this study, but a more absolute statement can be made once the enthalpy's of eutectic systems elements are better understood.

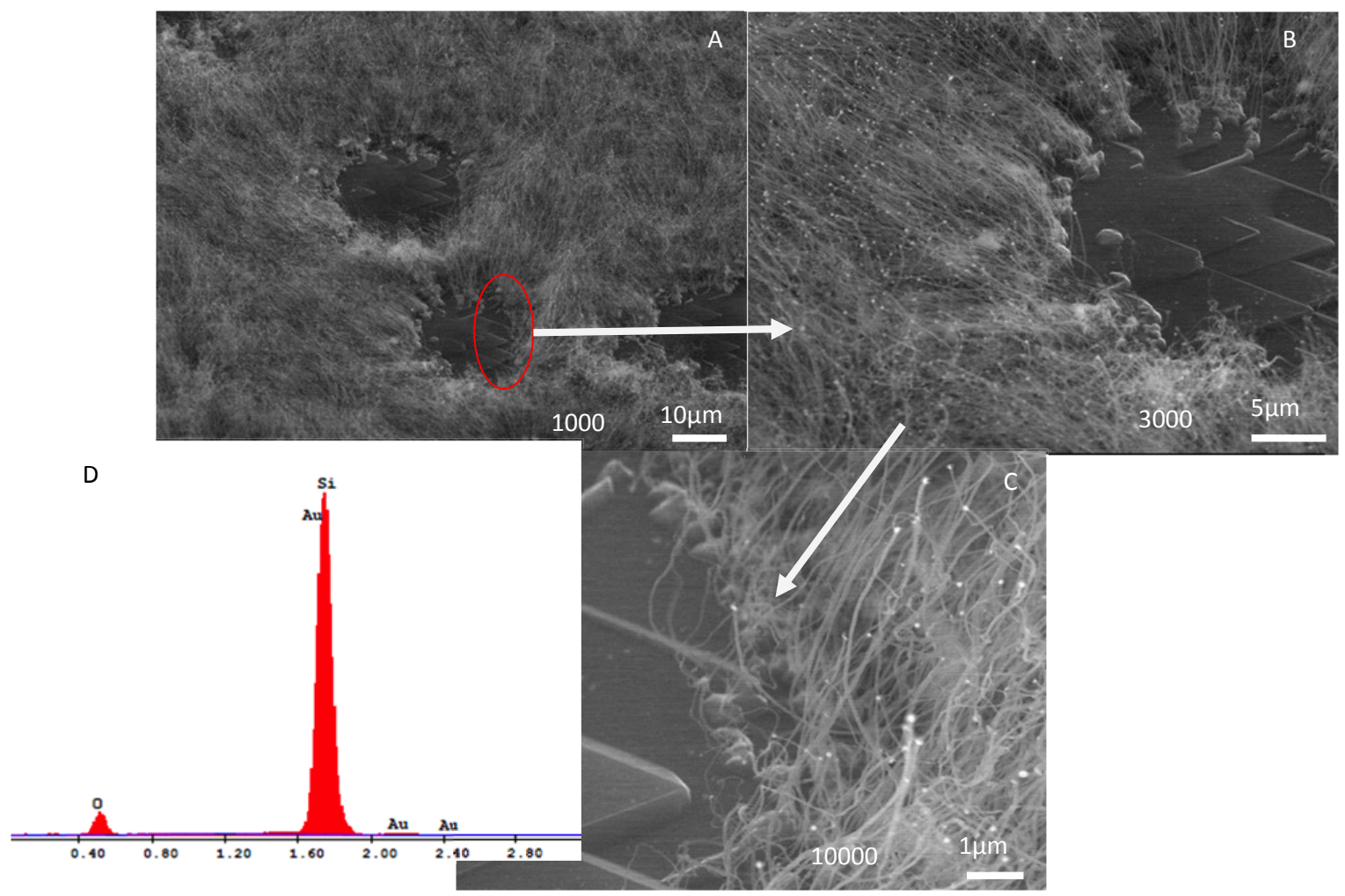

Figure 12. SEM composite and EDAX of area prepared with top carrier layer place over substrate nanowire growth on carrier substrate a) Aligned growth, b) 3000 magnification C) 10K magnification, and D) EDAX of area confirming presence of $\mathrm{Si}, \mathrm{O}$, and $\mathrm{Au}$. 


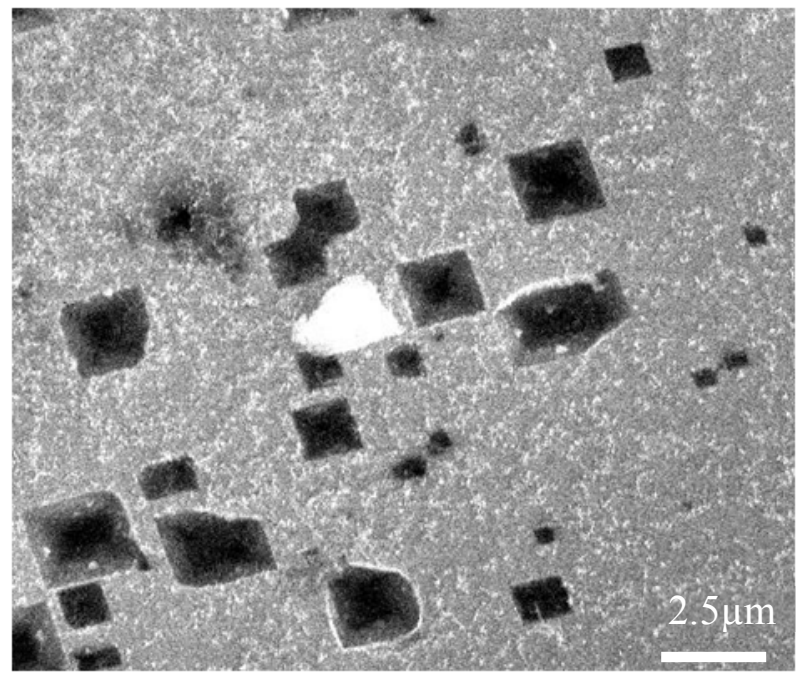

Figure 13. Silicon capping layer with etch pits on surface, evidence of $\mathrm{Si}$ dissolution on this surface instead of growth substrate.

\section{Conclusion}

Silica nanowire synthesis was achieved, while preserving the growth substrate. The growth substrate was left intact with indented ridges as primary surface marks. The expected etch pits were not present, this was due to the use of a capping layer (bare Si wafer) above the growth substrate. This capping layer served as the primary source of silicon, thus preventing etch pitch formation on the growth substrate. These results were realized on a $\mathrm{Si}$ $<111>$ oriented substrate. Silica nanowires grown on these surfaces were aligned with metallic tips intact and morphologies similar to those grown on $<100>$ substrates in the previous section. This technique has demonstrated that aligned wires can be produce via VLS without the use of vacuum or toxic silicon precursors. Additionally, the growth substrate surface was preserved from significant structural damage, which directly implies that this technique could be used to integrate nanowires into microscale devices where the growth substrate should be left unaffected morphologically from the growth tor annealing process. 


\section{Chapter 5}

\section{Metal Catalyzed Silica Nanowires for Biosensors Applications}

\section{Introduction}

After observing and understanding nanowire growth kinetics needed to produce structures of proper orientation and elemental composition, the next step in fabrication of a complete device was integration of nanowires into a micro-scale sensing structure. An ideal structure(figure 1) would be intact both with pre-existing and integrated structures properly formed and integrated. So far, the Vapor Liquid Solid growth method has been shown to be effective at synthesizing nanostructures in a simple and repeatable form without destruction the targeted growth substrate.

For demonstration of this integration technique a microelectrode interdigitated sensing structure has been chosen. These interdigitated electrodes (IDE's) were chosen due to their demonstrated use as sensors in surface acoustic wave (SAW), biological and redox applications with potassium ferro/ferrihexacyanid[77, 78]. Additionally, this structure has been optimized by Alexander et al for an impedance analysis of epithelial breast cancer cells, using an optimized IDE spacing to improve bio-impedance response, sensitivity and improve signal to noise characteristics[79]. The integration of these nanostructures into this preexisting and demonstrated device could yield promising result in terms of increased sensing area, from utilization of nanowire stems and interdigitated planar surface. However, such integration has presented challenges that involve metallurgy (IDE composition), fabrication (barrier layer placement/removal), nanowire integration (annealing temperature/time) and analytic toxicity due to nanowire incompatibility. 
To address the above mentioned challenges silica nanowires were selectively placed in a space measuring $5 \mathrm{um}$ in width and the length of one the electrode fingers. Using the VLS process catalyzing material e.g. a metal $(\mathrm{Pd}$ and $\mathrm{Au})$ were placed in the confined area annealed to produce nanowires. Upon integration, the optimized IDE structure was preserved after high temp annealing (nanowire integration) and was found to electrically conductive. Impedance Spectroscopy measures of Anti-Prostate Antigens were used to eclectically verify the presence of the silica nanowires within the structure and indicated by a resistive change upon nanowire integration. A study is presented below addressing the issue of nanowire toxicity. Specifically, the effect of prolonged silica nanowire exposure to cellular media containing epithelial breast cancer cells has been investigated. This is fundamental for the use of future applications that may involve the placement of such nano-micro hybrid devices in vivo biosensors.

In this chapter the topics of silica nanowire cytoxicity and microscale integration are presented and discussed. The discussion below highlights in detail the single step methodology used in integrating silica nanowires into the 5um spacing on the interdigitated microelectrode structures (IDE). The Vapor-Liquid-Solid growth process was used to facilitate this growth. Additionally, analysis of breast cancers cells in nanowire containing media is presented below to verify the biocompatibility of a microscale device containing integrated nanostructures for use with human biological tissue. 


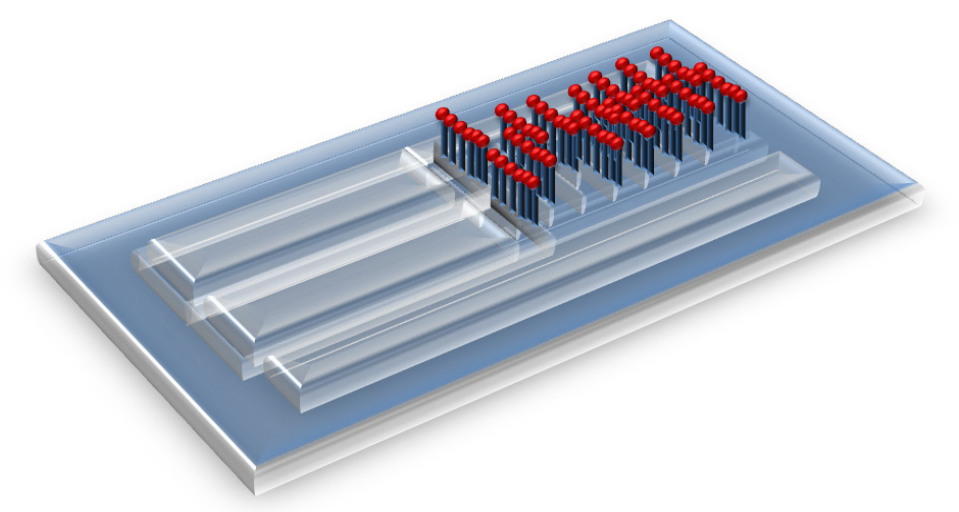

Figure 1. Ideal sensing device with vertically aligned silica nanowires integrated into interdigitated microelectrode structure.

\section{Optimized growth and integration of silica nanowires into interdigitated microelectrode structures for bio sensing}

\section{Experimental}

The silica nanowire based biosensor was developed on an interdigitated microelectrode structure. A schematic of the IDE is shown in Figure 2. The electrode device has two working electrodes and a comb like pattern along the length of structure. The comblike structures form the sensing area. IDE's are preferred structure for electrochemical biosensors, due to the ability to amplify the detection, which is strongly dependent on the inter-electrode separation. Although, interdigitated electrodes with an electrode separation of less than one micrometer are desired for maximum signal amplification, due to lithographic limitations, the microelectrodes have been designed to have a $5 \mu \mathrm{m}$ separation. This distance will facilitate the interaction of nanowires with the biomolecules and result in a fast detection of PSA. 


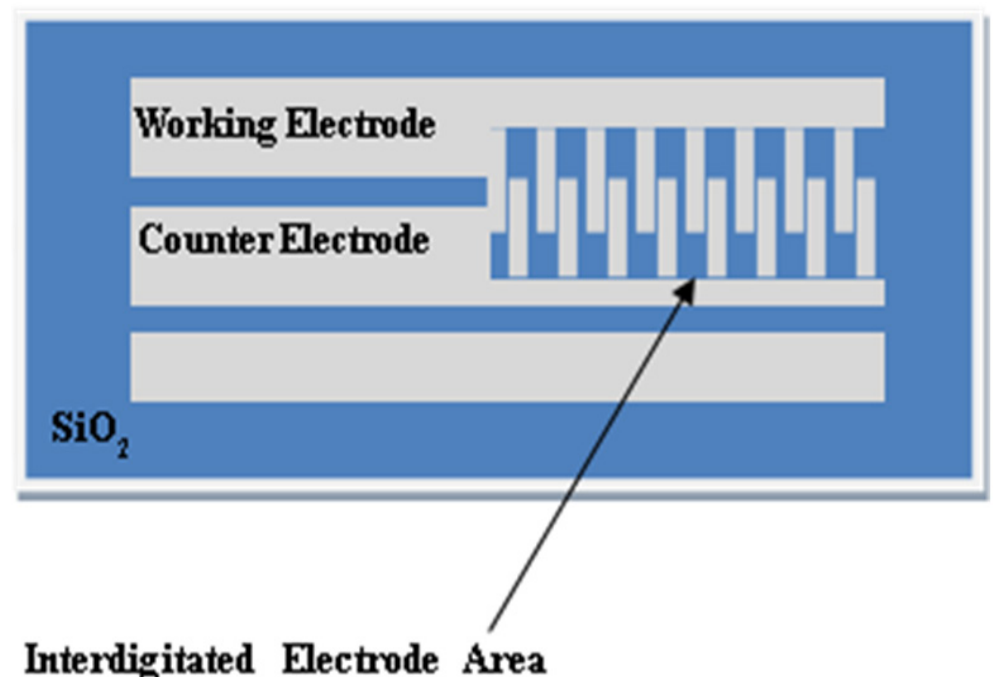

Figure 2.Schematic drawing of the interdigitated microelectrode device structure used for nanowire integration and sensing.

The interdigitated microelectrode devices were fabricated on a 4" silicon substrate. Figure $1 \mathrm{~b}$ illustrates the process sequence used to fabricate the electrodes. Initially, the silicon substrate was thermally oxidized to grow a $300 \mathrm{~nm}$ thick oxide layer. This is grown to prevent electrode contact with the silicon wafer surface. Direct contact with the substrate could possibly cause and electrical short, bridging the electrodes and the silicon surface and possibly disrupt the electrical reading of the device. After oxidation the wafers were patterned with a negative resist and lithographed to reveal spacing for electrode development on the silicon wafers. The microelectrodes and IDE were formed with a $20 \mathrm{~nm}$ Ti adhesion layer and $150 \mathrm{~nm}$ Pt deposition for electrode formation. Pt also does not readily oxidize in ambient environments; this is of concern, due to ambient exposure of device during machine transfer between fabrication steps and would help to ensure structural integrity throughout the fabrication /nanowire synthesis process. Both metals were deposited without breaking vacuum using electron beam deposition. Following deposition the wafer containing these devices was placed in metal etchant for liftoff, to remove resist and metal to reveal the IDE 
microelectrode pattern. Finished devices contained 24 individual sensing devices, each with a structure composed of 35 interdigitated fingers measuring $10 \mu \mathrm{m}$ pitch and $5 \mu \mathrm{m}$ width, and providing a total sensing area of $500 \mu \mathrm{m}$. The devices were cleaned with deionized water and nitrogen dried, and then diced as individual die for nanowire integration.

Figure 3a-c is an image of the fabricated interdigitated electrode device and flow diagram for nanowire integration. To perform the next critical step, silicon oxide use to separate the electrodes from substrate surface needed to be removed and Au would need to be deposited in these areas. The deposited Au would serve as a catalyst for nanowire growth, but only on those regions that where $\mathrm{Au}$ and silicon were in contact with each other. A bath of buffered oxide etchant of 5:1 composition was mixed and used to selectively remove silicon oxide from the wafer surface. Then, a thin layer of catalyst $\mathrm{Au}(5 \mathrm{~nm})$ was deposited by evaporation technique. The thin metallic layer between the electrode fingers will serve as the seed layer (catalyst) for nanowire growth. This technique also serves as a nanowire growth deterrent on the electrode, thus controlling the growth location. Silica nanowires were produced by high temperature annealing using the Vapor-Liquid-Solid mechanism $[8,36]$ Silica nanowire integration optimization was performed to determine the optimal conditions (annealing temperature and time) that would yield the densest amount of nanowires without damaging microelectrode structure. 
$\mathrm{B}$

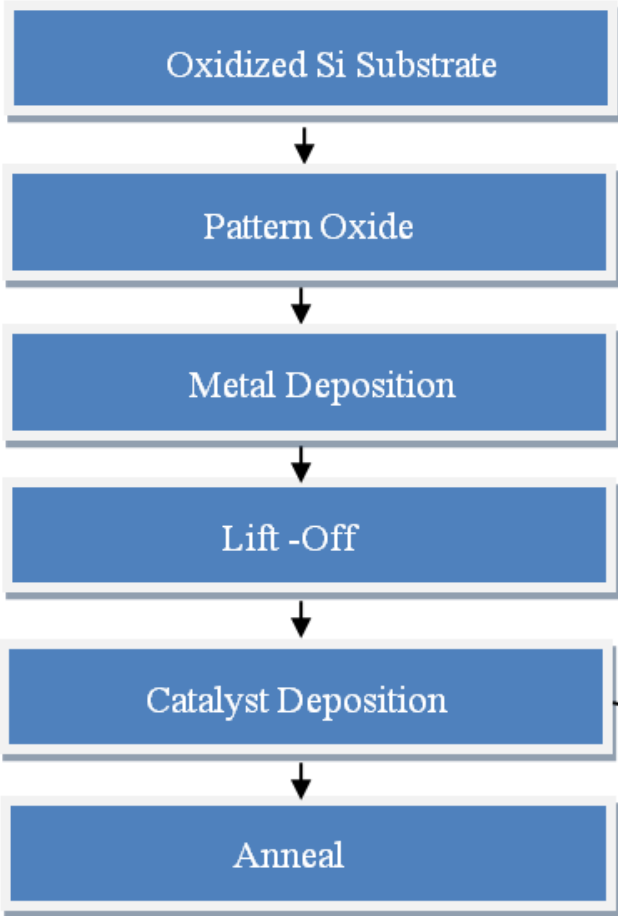

A

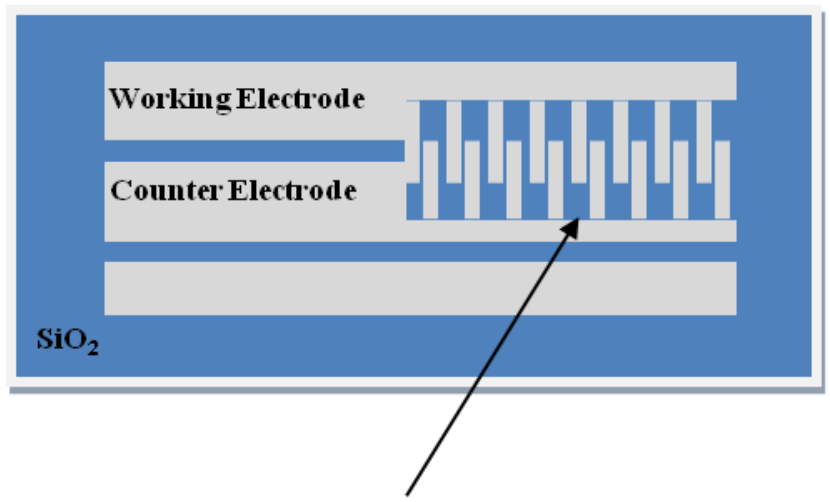

Inter digitated Electrode Area

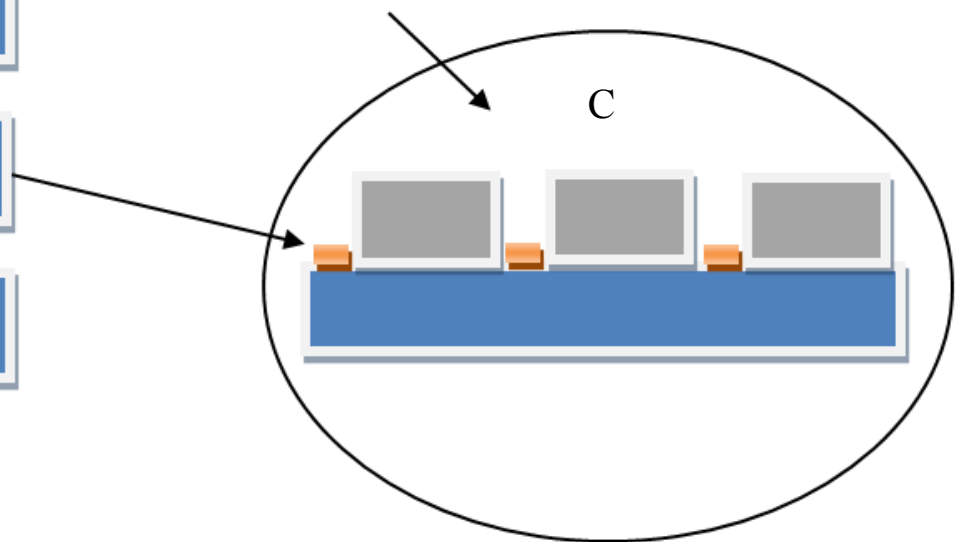

Figure 3a-c. Schematic and flow diagram illustrating the Microelectrode/Nanowire integration process a) Fabricated interdigitated microelectrode device, b) Process flow of electrode fabrication and nanowire integration, and c) inset of interdigitated electrodes showing catalyst placement for nanowire growth.

For nanowire synthesis, the devices were placed individually in an oxidation furnace.

The furnace was heated to $1050 \mathrm{C}$ in the presence of Argon ambient flowing at $25 \mathrm{sccm}$. Argon was used as a purging agent to remove contaminating species from the furnace chamber and as a flow gas to support nanowire formation. A growth time of $60 \mathrm{~min}$ was selected from previous experimentation. It was determined based on growth trials and weight estimates/modeling that $60 \mathrm{~min}$ at a temperature of $1050 \mathrm{C}$ would yield nanowires of approximately $100-125 \mathrm{~nm}$ in diameter and length of several microns. This size is needed to allow for future functionalization of wires and for this purpose they are customized as such. Wire growth was performed with the Si capping layer and without. 


\section{Antibody Immobilization}

Interdigitated microelectrode devices with nanowires were successfully grown inbetween the electrodes and functionalized with anti-PSA for PSA detection. The IDE's were pre-cleaned and exposed to the PSA antibody solution for surface modification. For modification, the PSA antibody solution $(10 \mathrm{mM}, \mathrm{pH} 7.0,0.9 \% \mathrm{NaCl})$ containing $5 \mathrm{mM}$ was placed on the devices and allowed to incubate for 24 hours. The sensor was then washed thoroughly with PBS (10 mM, pH 7.4) to remove any unbound biomolecules on

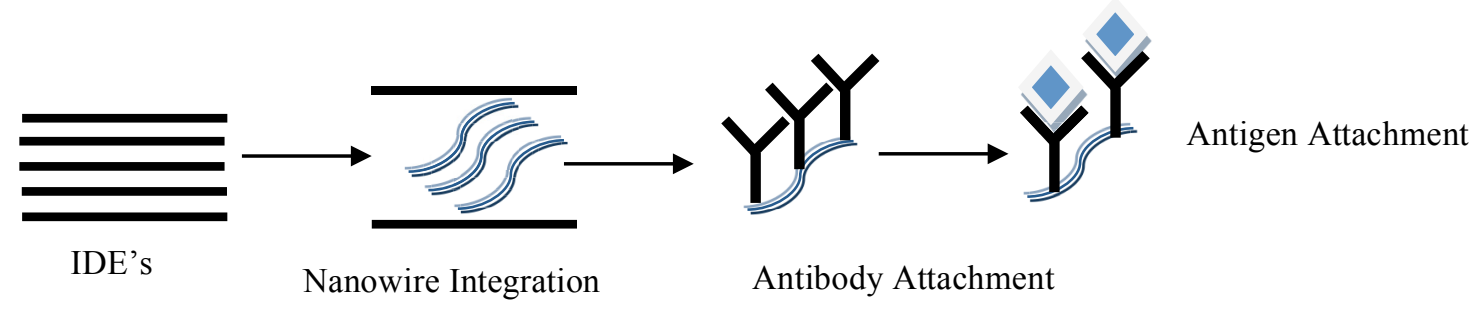

Figure 4. Antibody immobilization to form the biosensor. (Reproduced with Permission [2] )

the electrode surface and then incubated with a solution containing prostate specific antigens. The fabricated bio-electrodes were characterized using the electrochemical impedance technique at each solution/chemical addition step to determine antibody binding and antigen detection, respectively. Figure 4 schematically illustrates the antibody immobilization to form the biosensor. 


\section{Results and Discussion}

Structural Characterization

The nanowires were characterized using scanning electron microscope (SEM) and Transmission Electron Microscope (TEM) to determine the structure of the nanowires as well as the microelectrode integrity.
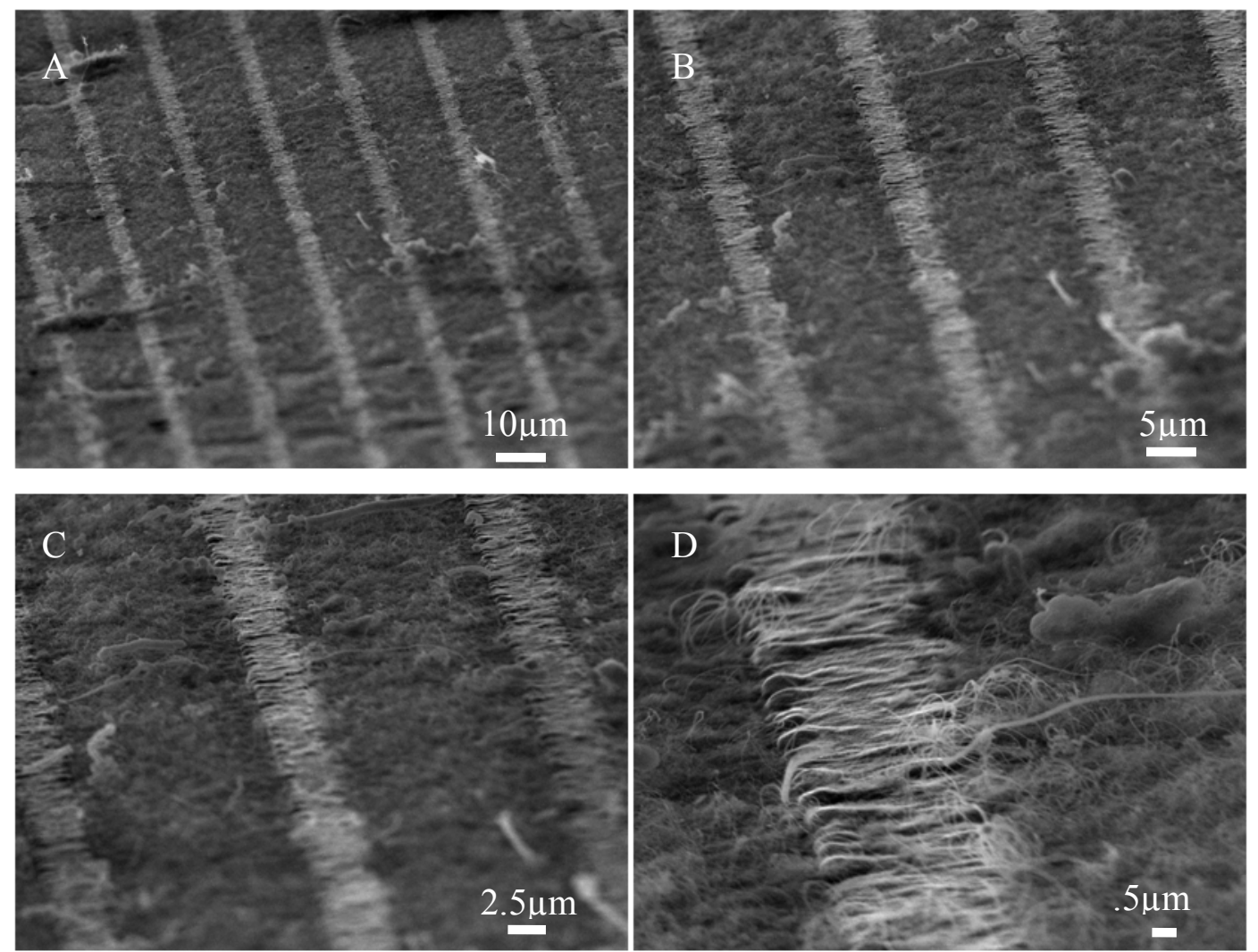

Figure 5. Angled view of interdigitated microelectrode with dense nanowires grown between the working electrodes a) $1 \mathrm{k}$, b) $2 \mathrm{k}$, c) $3 \mathrm{k}$, and d) $10 \mathrm{k}$ magnifications.

Figure 5 shows an SEM micrograph of the silica nanowires integrated into an interdigitated structure. The nanowires are randomly oriented and tangled, as expected on a $\mathrm{Si}<100>$ wafer and found to be confined between electrodes. Nanowires are dense and the 
substrate floor is covered. A capping layer (wafer cover) was not used, so it is understood that the growth substrate itself is the supplier of vapor phase $\mathrm{Si}$, which would lead etch pits o

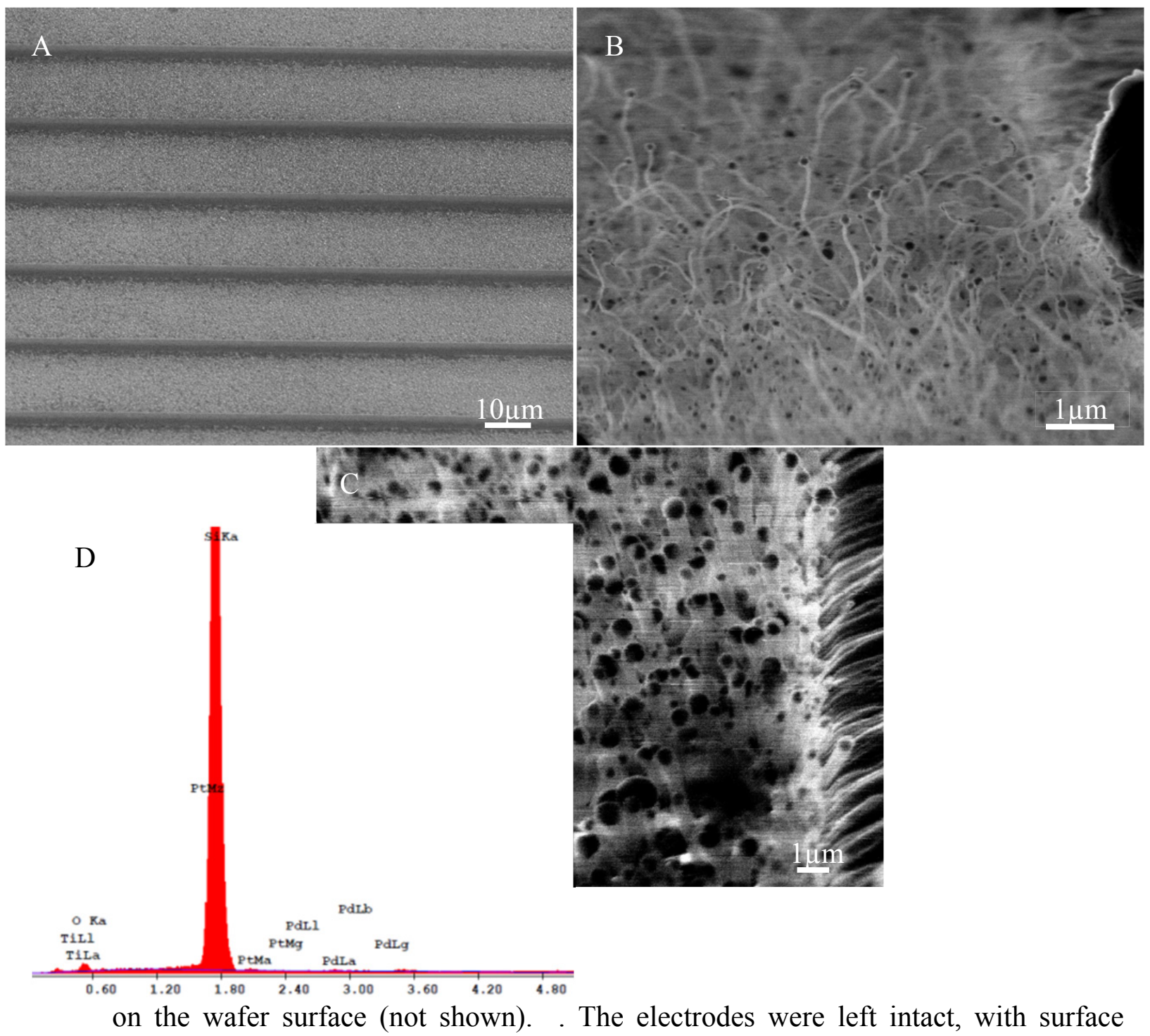

rippling noticeable, attributed to the some annealing effects. Electrical continuity of interdigitated fingers was found to be intact. 
Figure 6a-c are micrographs of the silica nanowires successfully grown between Pt IDE's with Si capping layer placed a distance of $500 \mu \mathrm{m}$ from electrode surface. The nanowires are more vertically aligned than those grown without the capping layer. The morphologies of the structure are identifiable such the prominent metallic tips at the ends the nanowire stems. Nanostructure growth was confined strictly between electrodes without any noticeable over hang, as observed with on surfaces grown without capping layer. Additionally, the electrode surface was left intact with a smooth planar top surface, rough portion were noticed on the sidewalls of the electrodes. EDS of the area confirmed the presence of expected elements, those that the electrode is composed of and those within the nanowires. Figure 7 is a TEM image showing a single intact nanowire. Figure $7 \mathrm{c}$ shows the granularity of the nanowires confirming amorphous structure and additional analysis revealed the composition of the wire stem to be composed of $\mathrm{Si}_{2}$ and $\mathrm{O}$. XRD in figure 8 confirms the observed peaks in EDS (Chapter 4) and corresponds to reflection planes of $\mathrm{SiO}_{2}$ and correlates with those reviewed in literature. The broad XRD peaks are attributed to the nanoscale formation of wires. 


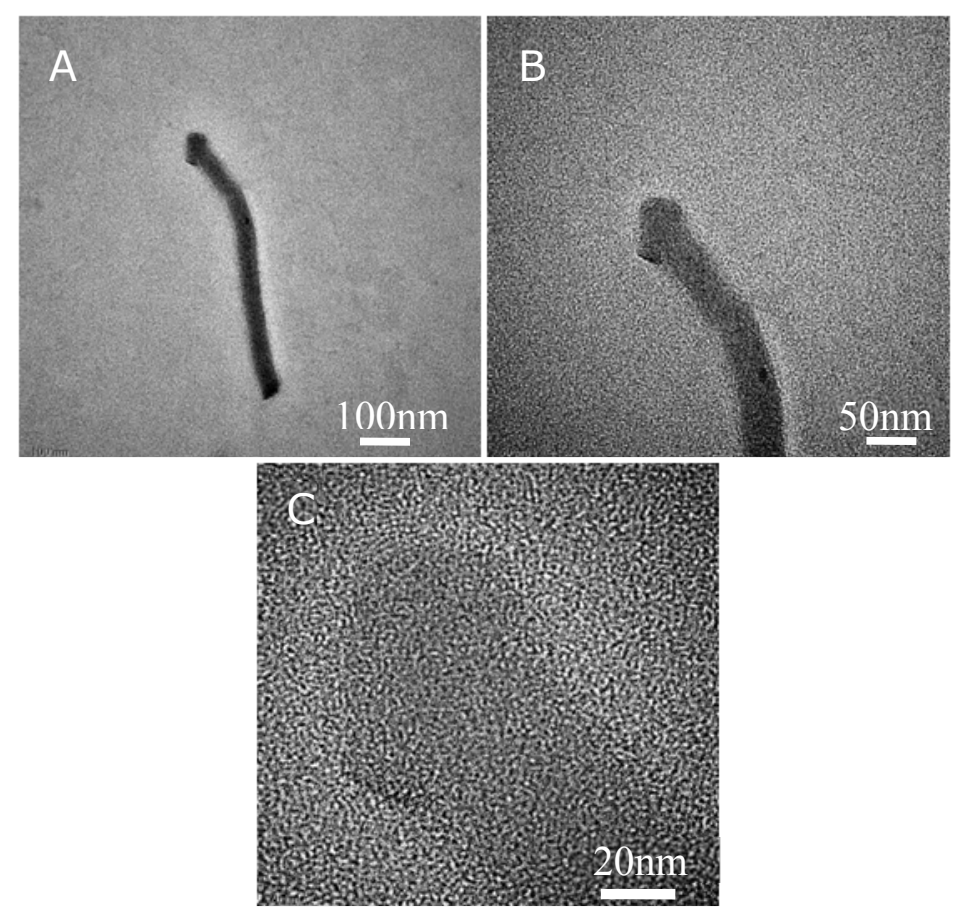

Figure 7 TEM of a nanowire revealing its composition to be amorphous and with a diameter of approximately $50 \mathrm{~nm}$.
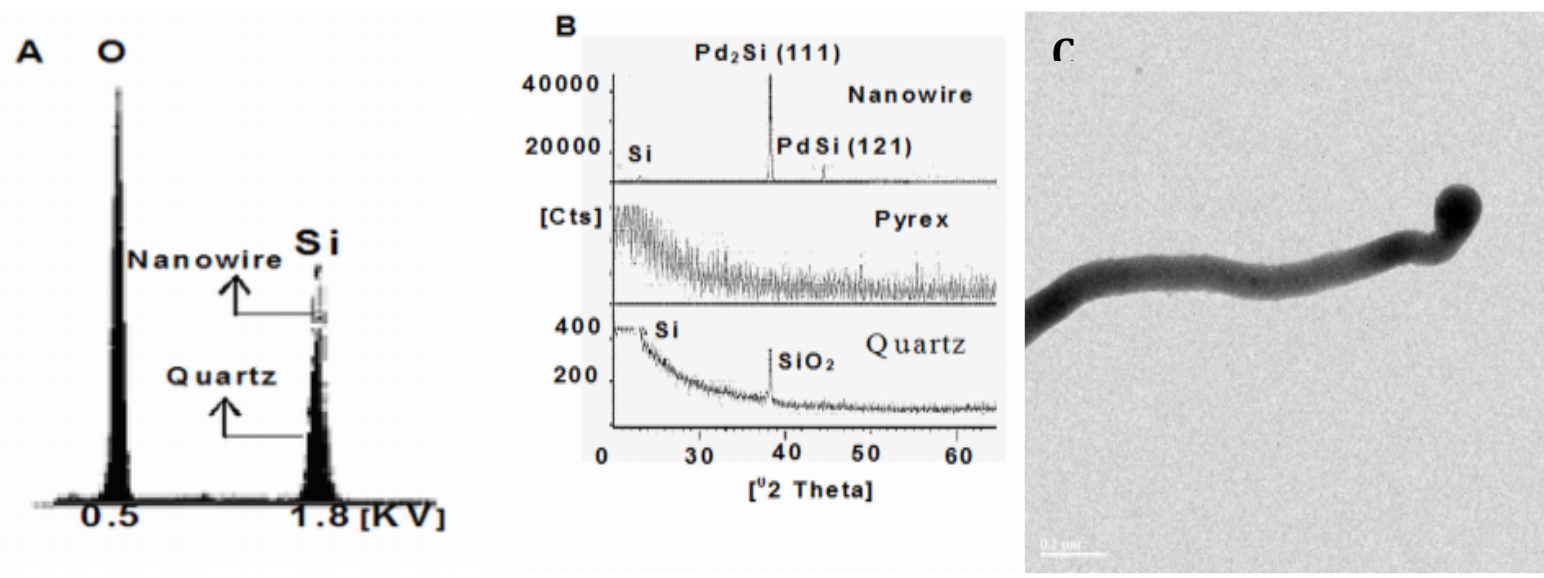

Figure 8. XRD confirming the nanowire composition be of $\mathrm{Si}, \mathrm{O}$ and $\mathrm{Pd}$. Scale bar in $8 \mathrm{c}$ is $.3 \mu \mathrm{m}$. [Courtesy Praveen Sekhar] 


\subsection{Bio-Electrical Characterization}

Nyquist plots of impedance spectra were plotted to study (a) the change in the charge transfer resistance at the biosensor interface after anti-PSA binding with and without the nanowires on IDE, (b) the change in charge transfer resistance with antigen attached to blank IDE and IDE with nanowires. All EIS spectra were recorded in PBS (10 mM, pH 7.4) containing $5 \mathrm{mM}$ as a redox probe.

Figures 8 and 9 show Nyquist plots of impedance spectra measured on the interdigitated electrodes without and with nanowires, respectively. Interdigitated microelectrode device without nanowires (Figure 8) shows an increase in charge transfer resistance (diameter of semicircular part) from $856 \Omega$ (curve (i)) to $1679.2 \Omega$ (curve (ii)) after anti-PSA binding on blank IDE's. This indicates the binding of antibody on the device surface. Further the increase of charge transfer resistance to $4686.50 \Omega$ as showing in curve (iii) reveals successful binding of antigen onto antibody. The increase is attributed to the fact that binding of PSA create extra insulating layer on electrode surface thus resulting in hindrance to charge transfer from solution to electrode and hence increasing the charge transfer resistance.

Figure 9 shows the Nyquist results with nanowires grown in place with IDE's. In comparison to the blank IDE as shown in Fig. 8, curve (i) - sample with no wires; higher initial charge transfer resistance of $1366 \Omega$ is observed in Fig. 9, curve (i). With antiPSA bound to the silica nanowire, the contact resistance increases to $13975 \Omega$ as shown in Fig. 8 curve (ii). This large increase in resistance can be attributed to increased binding of anti-PSA on large sensing surface area provided by the integration of the silica nanowires. Lastly, an 
increase to $19464 \Omega$ with antigen attachment is observed as shown in Fig. 8 curve (iii). This reveals the intact activity of bound antibodies in silica nanowire network.

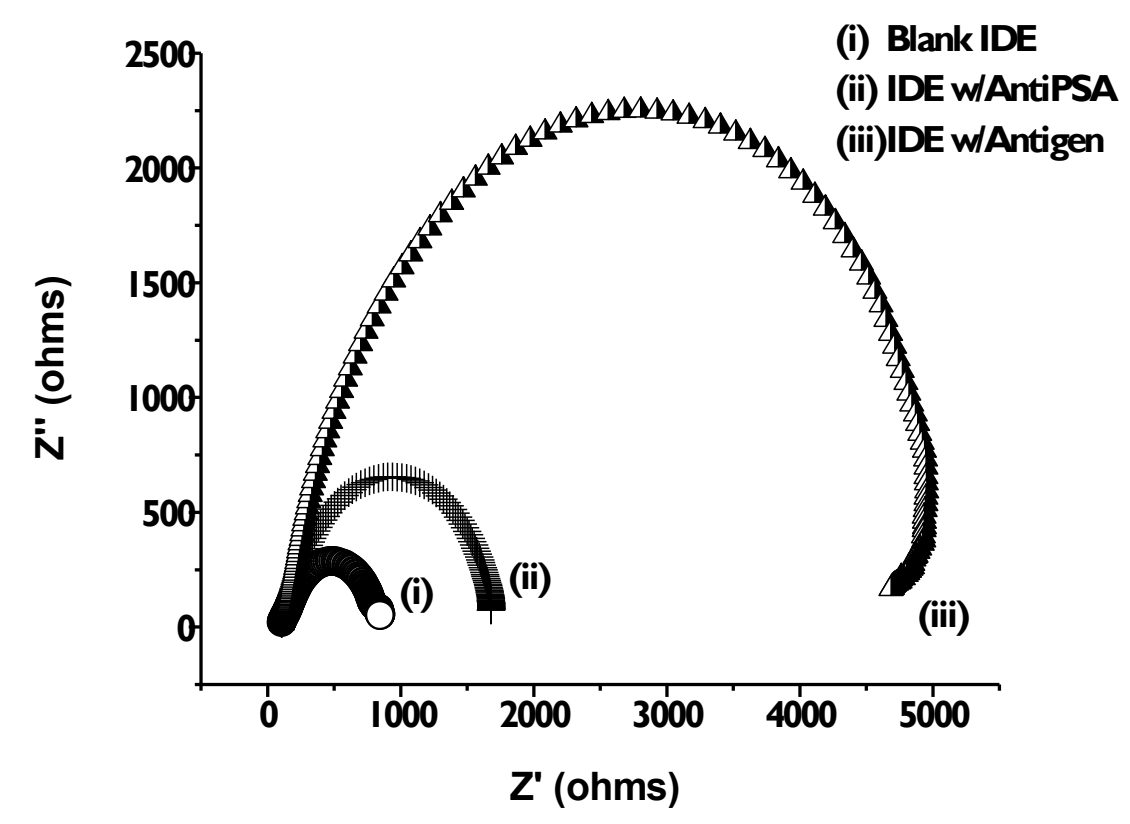

Figure 8 Nyquist plots of (i) Blank IDE, (ii) IDE with AntiPSA, and (iii) IDE with Antigen (Reproduced with Permission[2] )

For each of the curves presented above, an increase is resistance was observed with each testing phase and solution addition. This increase in resistance was expected due to the insulating nature of the antibodies and antigens. Similarly, previous work with these electrodes reported by Arya et al [80]revealed similar resistance trends from antibody/antigen attachment. The resistance change after the antiPSA attachment to the nanowires was unexpected. Based on work reported by Murphy-Perez et al [81] antiPSA attachment should have caused a change on the order of $4000-6000 \Omega$. This could be due to the partial detachment of the nanowires causing this substantial increase in resistance. 


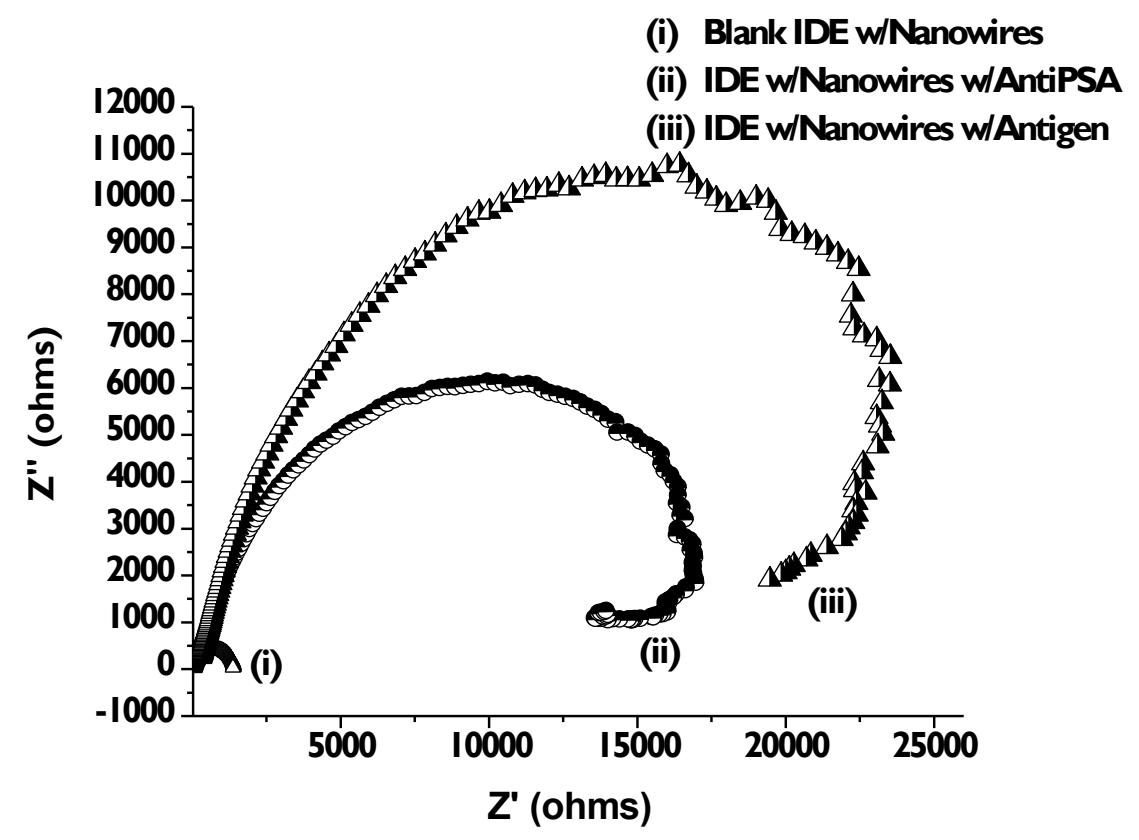

Figure 9 Nyquist plots of (i) Blank IDE with nanowires, (ii) IDE with AntiPSA/nanowires, and (iii) IDE with Antigen/nanowires (Reproduced with Permission[2])

\section{Conclusion}

In summary, nanowires have been grown successfully and "in place" on a working substrate using the VLS method. TEM and EDAX data indicate wires were amorphous and the metal catalyst used for growth was distributed along the length of the wires, respectively. SEM micrograph revealed integration of nanowires in microelectrode structures of IDE's. Conductivity studies suggested electrically intact properties of microelectrodes. EIS spectra taken after nanowire/IDE integration showed a noticeable increase in resistance after the successful attachment of antibodies and antigens. This observable increase in resistance will make it possible to further functionalize these devices for attachment with self-assembled monolayer's (SAM's) for enhanced detection of biologically important analyte and cancer biomarkers. 


\section{Quantitative impedance analysis of nanowires and cancer cell}

\section{Experimental}

\section{Nanowire synthesis}

Silica nanowires (NW's) were grown using the vapor liquid solid method[8, 9] on 4" silicon wafers. Initially, the Si substrates were cleaned with buffered oxide etchant to remove any native oxide formed on the surface and a $5 \mathrm{~nm}$ thin palladium was deposited via e-beam evaporation as growth catalyst. An open-tube furnace was used for growing the nanowires, which was heated to $1050{ }^{\circ} \mathrm{C}$ and purged with argon during the entire process. Argon served as a carrier gas for vapor phase silicon and to remove contaminating species from furnace tube. The Pd-sputtered Si substrate was placed on a bare Si wafer and placed onto a quartz boat, which was loaded into the open-tube furnace. The sample was annealed for 60 minutes at $1050{ }^{\circ} \mathrm{C}$, to yield a dense formation of wires that would be suitable for characterization and cell testing. Figure 10a shows an SEM image of the nanowires grown with this process. Scanning Electron Microscopy (SEM) revealed the nanowires to have diameters on average of 100-200 $\mathrm{nm}$ in width and EDS verified wire elemental composition to be silicon and oxygen with palladium growth catalyst dispersed along the length of the wire. 


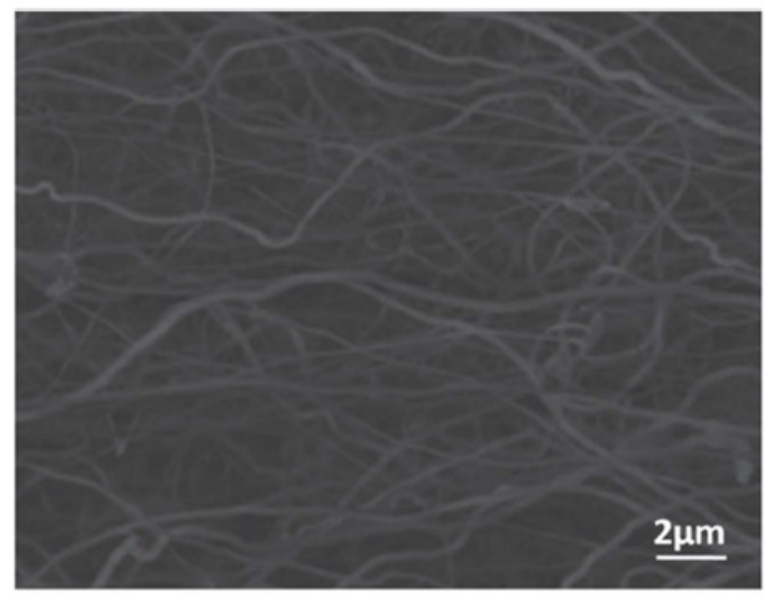

(a)

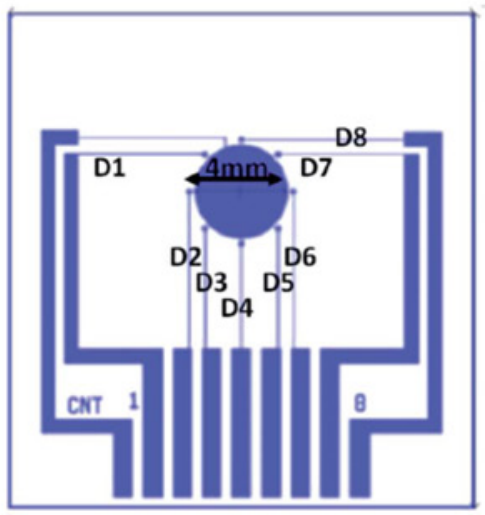

(b)

Figure 10 (a) SEM micrograph of silica nanowires annealed for 60 minutes at 1050 _C. (b) Electrode arrays were fabricated on a glass substrate from $\mathrm{Cr} / \mathrm{Au}$ using a standard lift-off procedure. A single counter electrode $(4 \mathrm{~mm})$ is placed in the center surrounded by eight working electrodes (D1-D8). (Reproduced with Permission[1])

\section{Microelectrode device fabrication and preparation}

Microelectrode arrays were fabricated to monitor monolayers of Hs578T epithelial breast cancer cells (ATCC: HTB-126). The electrode geometry, shown in Figure 10b contains a circular array of 8 working microelectrodes (diameter $=250 \mu \mathrm{m}$ ) with a single counter electrode (diameter $=4 \mathrm{~mm}$ ) in the center. The counter electrode is made much larger than the surrounding working electrodes to ensure that the impedance of the cellular monolayer dominates the measured impedance as opposed to impedance of the electrodes. Electrodes were designed to increase the spatial resolution over currently available electrode designs. By incorporating a circular array of working electrodes, multiple areas of a single culture are monitored during a single experiment. In addition to increased spatial resolution, this incorporates measurement redundancy, an essential requirement for generating statistically significant results. The microelectrode arrays were patterned on a 4" glass wafer using conventional lithography technique followed by thin film deposition of $\mathrm{Cr}(15 \mathrm{~nm})$ and 
$\mathrm{Au}(60 \mathrm{~nm})$ to define the microelectrodes and contact pads. Later, the electrode tracks were insulated using SU-8 and cloning cylinders were attached to the devices using SU-8 to hold the culture medium and cells covering the electrodes. The cloning cylinders were reinforced and leak-proofed using high vacuum grease. The fabricated devices were cleaned with isopropanol, deionized water, and then sterilized by dry heat sterilization for 90 minutes in an oven at $170{ }^{\circ} \mathrm{C}$.

\section{Cell culture and inoculation}

Hs578T epithelial breast cancer cells (ATCC: HTB-126) were cultured in Dulbecco's Modified Eagle Medium (DMEM) supplemented with 10\% Fetal Bovine Serum (FBS), 0.01

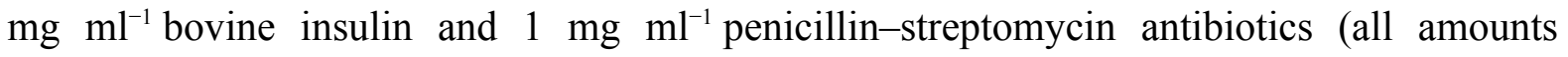
indicated are final concentration). During culture and experiments, cells were kept in a humidified atmosphere (incubator) at $37{ }^{\circ} \mathrm{C}$ and $5 \% \mathrm{CO}_{2}$. Cells were grown to $90 \%$ confluence and then harvested using a 25\% trypsin/EDTA solution. Viable cells were then removed to a separate flask and counted using a hemocytometer and the tryphan blue exclusionary technique. Prior to cell seeding microelectrode surfaces were pre-treated with 0.1\% type B bovine gelatin solution (Sigma). Microelectrode arrays were incubated with gelatin solution for 30 minutes and then rinsed with complete culture medium prior to cell seeding. This surface treatment improved cellular attachment to electrodes for experimental impedance monitoring. Cells were then seeded onto impedance devices at a density of $\sim 2 \times$ $10^{4}$ cells per device. Microelectrodes were connected to the switching circuit located inside an incubator and monitored for 24 hours or until baseline measurements stabilized. 


\section{Nanowire preparation and inoculation}

Pd-catalyzed NWs were scraped from the growth substrate and weighed to be approximately $200 \mu \mathrm{g}$. In order to avoid contamination, NWs were then sterilized in an oven at $170{ }^{\circ} \mathrm{C}$ for 90 minutes. Sterilized NW's were then added to $1 \mathrm{ml}$ of sterile growth medium (DMEM) and dispersed using ultrasonic agitation for 1 minute. A stock solution of $\mathrm{NW} / \mathrm{DMEM}$ of $200 \mu \mathrm{g} \mathrm{ml}{ }^{-1}$ was refrigerated at $4{ }^{\circ} \mathrm{C}$ until needed for further dilution and cell inoculation. Cells were inoculated with nanowire-medium solution with final concentrations of $1 \mu \mathrm{g} \mathrm{ml} l^{-1}, 50 \mu \mathrm{g} \mathrm{ml}^{-1}$, and $100 \mu \mathrm{g} \mathrm{ml}^{-1}$.

\section{Impedance measurements}

An Agilent 4294A Impedance Analyzer was used to make impedance measurements. Measurements were recorded using a $10 \mathrm{mV}$ peak to peak voltage over a frequency range of $100 \mathrm{~Hz}$ to $1 \mathrm{MHz}$ and were kept in an incubation chamber at a temperature of $37.1^{\circ} \mathrm{C}$ and an atmosphere consisting of $5 \% \mathrm{CO}_{2}$ to stabilize the environment for continuous cell growth. Impedance measurements were controlled remotely using an HP desktop computer with Labview software and an in-house switching circuit capable of monitoring 4 devices in parallel. In order to provide electrode functionality, baseline measurements were recorded using DMEM growth medium prior to cell seeding. Damaged electrode connections, due to residual photoresist or scratched electrode contacts, were discarded at the end of the baseline experiment. 
Next, cells were seeded onto devices, and cellular impedance was monitored for 24 hours prior to inoculation with nanowires. Once cells reached $100 \%$ confluence and cellular impedance stabilized, cells were removed from the incubation chamber and varying concentrations of nanowire solutions $\left(0 \mu \mathrm{g} \mathrm{ml}^{-1}, 1 \mu \mathrm{g} \mathrm{ml}^{-1}, 50 \mu \mathrm{g} \mathrm{ml}^{-1}\right.$, and $\left.100 \mu \mathrm{g} \mathrm{ml}^{-1}\right)$ were then prepared and seeded into the devices as described in the previous section. Finally, cells were immediately returned to the incubation chamber and the impedance of each device was then recorded once per hour for a minimum of 24 hours for real-time monitoring of cellular impedance.

\section{Equivalent circuit modeling}

Internally designed Matlab programs and Excel (Microsoft) spreadsheets were used to calculate impedance magnitude and phase data recorded by the impedance analyzer. Initially, Bode plots were used to visualize magnitude and phase data calculated for each measurement on a specific device. In order to assess changes in the culture, a time plot was then created; monitoring the average $|Z|$ calculated at $96.49 \mathrm{kHz}$. This frequency is significant because it lies within the "beta dispersion" where the capacitive impedance contributed by the cellular membrane is highest $[82,83]$ making it a good indicator of cellular activity. Equivalent circuit modeling via Matlab was used to extract parameters for baseline measurements. Two different equivalent circuit models were used to fit data using the complex nonlinear least squares (CNLS) method. Initially, all data was fit to (Fig. 11a) denoting lack of cellular presence. $\mathrm{CPE}_{\mathrm{dl}}$ represents the double layer capacitance at the electrode interface and $R_{\mathrm{s}}$ represents the solution resistance. Data was next fit to (Fig. $11 \mathrm{~b}$ ) to detect cell 
presence. Fit accuracy was evaluated by sum of squared error (SSE); where, parameters extracted with the smallest SSE value were taken to represent the system.

\begin{tabular}{|c|c|c|c|c|}
\hline (D07) & Rs & CPEdl-T & CPEdl-P & Sum-Sqr \\
\hline D1 & $2.62 E+03$ & $1.97 \mathrm{E}-08$ & 8.16E-01 & 2.89E-01 \\
\hline D2 & $2.39 E+03$ & $2.01 \mathrm{E}-08$ & 7.99E-01 & $1.74 \mathrm{E}-01$ \\
\hline D3 & $2.45 E+03$ & 9.97E-09 & $8.45 E-01$ & $1.49 \mathrm{E}-01$ \\
\hline D4 & $2.20 \mathrm{E}+03$ & $1.13 E-08$ & $8.17 E-01$ & $1.13 \mathrm{E}-01$ \\
\hline D5 & $2.21 E+03$ & $6.91 E-09$ & $8.62 E-01$ & $1.44 \mathrm{E}-01$ \\
\hline D6 & $2.27 E+03$ & 7.07E-09 & $8.60 \mathrm{E}-01$ & $1.34 \mathrm{E}-01$ \\
\hline
\end{tabular}

(a)

\begin{tabular}{|c|c|c|c|c|c|c|c|}
\hline (D07) & Rs & CPEdl-T & CPEdl-P & Rcell & CPEcell-T & CPEcell-P & Sum-Sqr \\
\hline D1 & $2.07 E+03$ & 2.40E-08 & $8.16 \mathrm{E}-01$ & $6.07 E+03$ & 8.33E-08 & 5.96E-01 & $1.20 \mathrm{E}-02$ \\
\hline D2 & $2.05 E+03$ & $1.91 \mathrm{E}-08$ & $8.25 \mathrm{E}-01$ & $5.94 \mathrm{E}+03$ & 4.79E-09 & $7.84 \mathrm{E}-01$ & $1.21 \mathrm{E}-02$ \\
\hline D3 & $1.60 \mathrm{E}+03$ & $6.34 \mathrm{E}-09$ & $9.19 \mathrm{E}-01$ & $7.52 \mathrm{E}+03$ & $1.25 \mathrm{E}-07$ & $5.50 \mathrm{E}-01$ & $7.62 \mathrm{E}-03$ \\
\hline D4 & $1.64 E+03$ & 8.01E-09 & 8.77E-01 & $4.97 E+03$ & 6.17E-08 & $5.92 \mathrm{E}-01$ & $7.62 \mathrm{E}-03$ \\
\hline D5 & $1.60 \mathrm{E}+03$ & $6.33 \mathrm{E}-09$ & $8.96 \mathrm{E}-01$ & $4.91 \mathrm{E}+03$ & 4.16E-08 & $6.26 \mathrm{E}-01$ & $9.13 \mathrm{E}-03$ \\
\hline D6 & $1.67 \mathrm{E}+03$ & 7.14E-09 & $8.94 \mathrm{E}-01$ & $6.73 E+03$ & $3.65 \mathrm{E}-08$ & $6.33 \mathrm{E}-01$ & $1.33 \mathrm{E}-02$ \\
\hline
\end{tabular}

(b)

Normalized Impedance after Initial Seeding

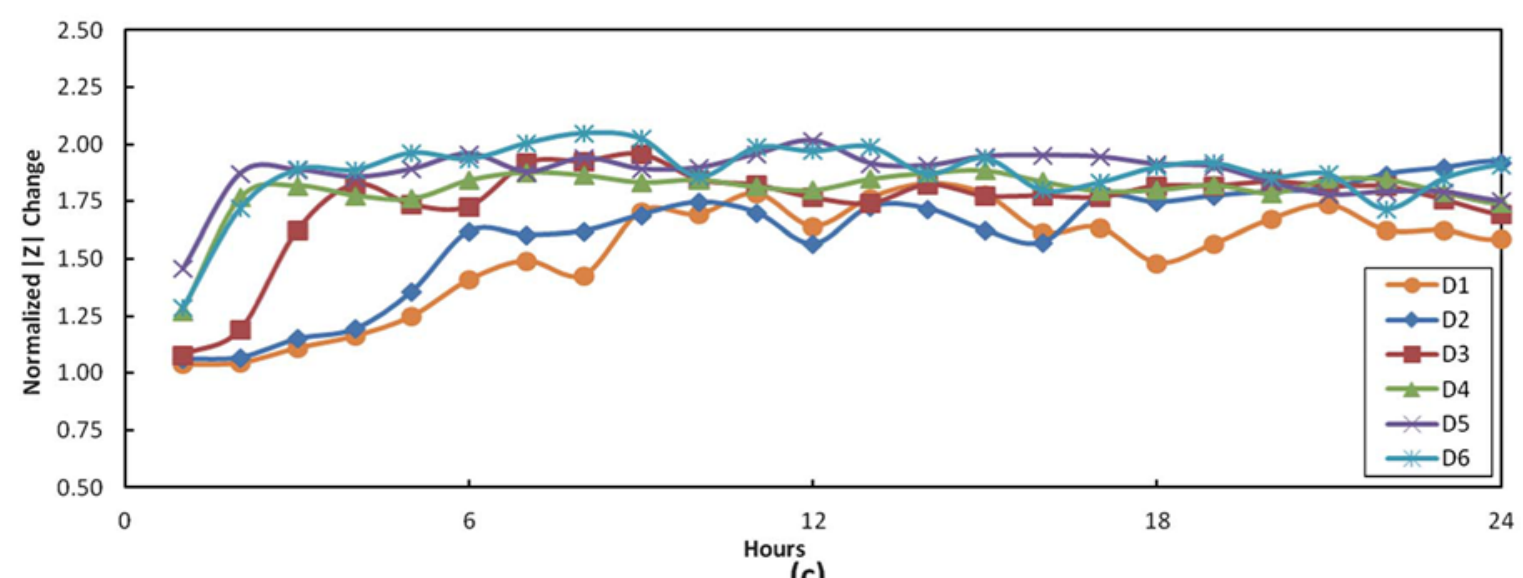

Figure 11. Baseline impedance spectra were first recorded using medium and then used to normalize cellular impedances for device D07. Measurements were fit to (a) to indicate lack of cellular presence and (b) for cellular presence. (c) Normalized $|\mathrm{Z}|$ at $96.49 \mathrm{kHz}$ were recorded for 24 hours. (Reproduced with Permission[1]) 


\section{Results and Discussion}

Baseline characteristics of cellular monolayer

To prove device functionality baseline measurements were first recorded for devices using only culture medium. This ensured electrode connectivity and provided a reference to compare with measurements of confluent cellular monolayers. The extracted parameters for the baseline (Fig. 11a) indicate that no cellular presence is detected within the culture and the impedance of the system is best approximated as a solution resistance in combination with the double layer capacitance. Baseline impedance values at $96.49 \mathrm{kHz}$ measured for each functional electrode (non-functional electrodes not reported) were recorded and used as normalizing factors for all future time plots of monitoring impedance changes. Values close to one indicate baseline characteristics and a very small change in impedance.

A time plot of data collected from the six independent working electrodes located on one device is shown in Fig. 11c, as an example of the increased spatial resolution made possible with this electrode design. It can be seen that at time zero, impedance measurements are very close to unity, indicating that while cells are present in the culture area, they have yet to spread over the electrodes and contribute to the impedance. Over the next 6 hours the impedance begins to increase rapidly due to cellular attachment to the substrate, eventually settling between an impedance increase of a factor of 1.5 and 2. It is important to note that the rate of increase varies over different electrodes, indicating differences in cellular coverage within the chamber. The measured impedance responses for electrodes D1-D6 on the electrode map correspond directly to specific spatial locations within the culture (see Microelectrode device fabrication and preparation section). From this data, it can be inferred that the majority of cells are initially attached to the area surrounding electrodes D4-D6. 
Cellular migration and division cause the impedance measured by other electrodes to rise when cells spread across them as time progressed.

After 6 hours, dedicated increases in impedance begin to taper off and measured impedance begins to fluctuate for each electrode indicating cellular confluence. The small fluctuations over time correspond to the micro motion of cells across the surface of the electrodes, as seen previously in prior studies[84]. Equivalent circuit parameters extracted 24 hours after cellular seeding (Fig. 11b) confirm cellular presence. The equivalent circuit model representing cell presence was chosen because SSE values were smaller than SSE values obtained with the alternate equivalent circuit model.

Cellular impedance response to nanowire inoculation

After 24 hours of incubation, devices were then seeded with concentrations of nanowires including: $0 \mu \mathrm{g} \mathrm{ml}^{-1}, 1 \mu \mathrm{g} \mathrm{ml}^{-1}, 50 \mu \mathrm{g} \mathrm{ml} l^{-1}$, or $100 \mu \mathrm{g} \mathrm{ml}^{-1}$. Fig. 12 shows the impedance response of the cancer cells to various concentrations of nanowires. A small increase after the first hour can be attributed to an initial decrease in impedance caused by being removed from the incubation chamber and restabilizing once returned to standard growth conditions. Over time, only controls and cells seeded with $1 \mu \mathrm{g} \mathrm{m} l^{-1}$ of nanowires remained attached and spread onto the electrodes. Cells that remained viable on the electrodes exhibited a stable impedance magnitude that continued to fluctuate in a small range due to cellular micromotion and activity. Cells inoculated with concentrations higher than $1 \mu \mathrm{g} \mathrm{ml}^{-1}$, in contrast, showed dedicated reductions in impedance magnitude immediately after initial seeding. After approximately 30 hours, cells exposed to the highest concentration of nanowires $\left(100 \mu \mathrm{g} \mathrm{m} l^{-1}\right)$ reached near baseline characteristics. This indicates that all cells had completely detached from the surface, resulting in cell death. 


\section{Average $|\mathrm{Z}|$ Response After Seeding}

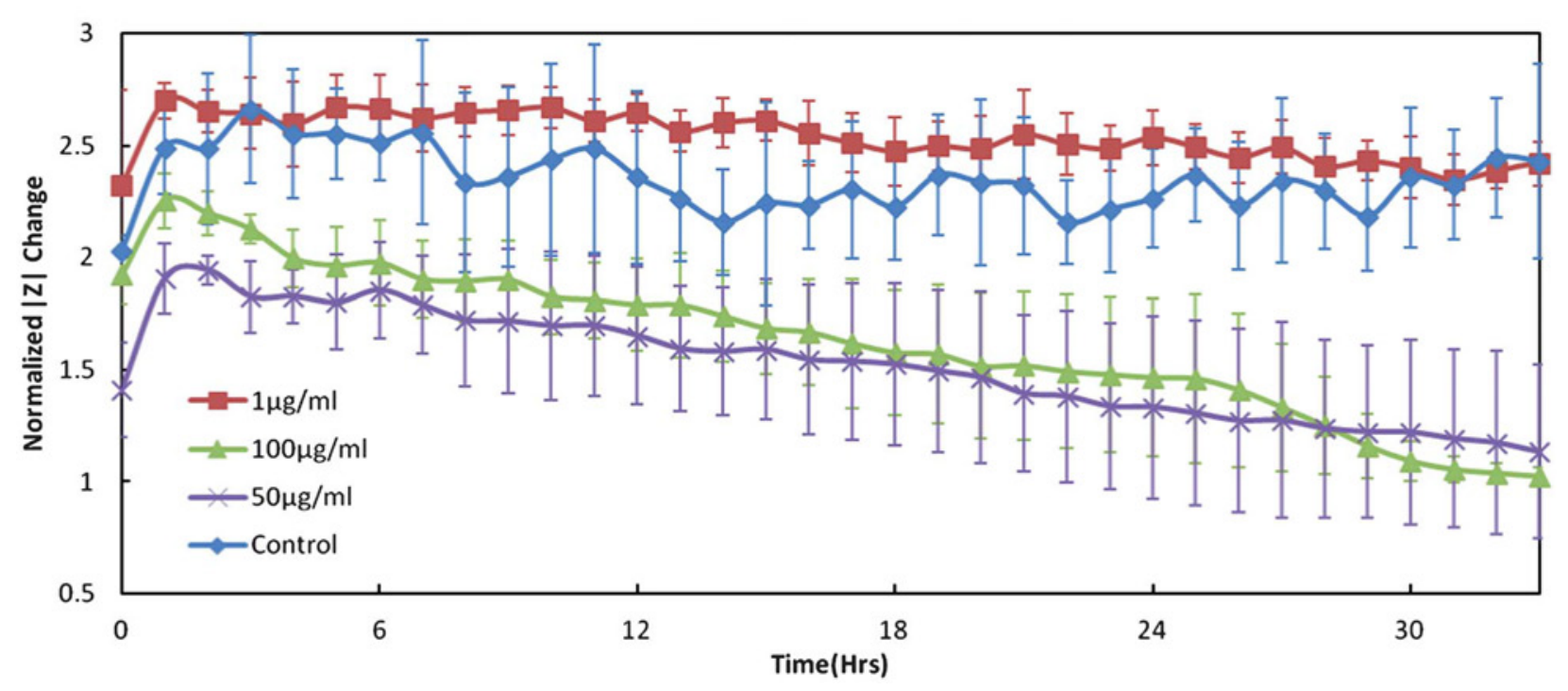

Figure 12. Average $|\mathrm{Z}|$ at a frequency of $96.49 \mathrm{kHz}$ recorded from each electrode on a single device with the standard deviation for a minimum of 24 hours. Lower concentrations $\left(0\right.$ and $\left.1 \mathrm{mg} \mathrm{ml}^{-1}\right)$ of silica $\mathrm{NW}$ maintain stable impedance while those with higher concentrations (50 and $100 \mathrm{mg} \mathrm{ml}^{-1}$ ) undergo decreased impedances. (Reproduced with Permission[1])

In this study, real-time impedance responses were used to monitor cellular bio impedance of the epithelial breast cancer cell line Hs578T in response to palladium catalyzed silica nanowires. Online cellular impedance measurements verified the results of previous studies indicating that a high concentration SiNW negatively impacts the viability of a confluent cellular monolayer.[85, 86] Julien et al.examined the mechanisms of cell death induced by exposing 3T3-L1, HeLa and Panc10.05 cellular cultures to silica nanowires and examining the effects at multiple time points. Cell counts taken using the tryphan blue exclusionary technique indicated that cells exposed to silica nanowires continued proliferative activity at the control concentration of $37 \mu \mathrm{g} \mathrm{ml}^{-1}$, compared to cells exposed to the apoptosis inducer cycloheximide $(\mathrm{CHX})$, which resulted in cell death. Further inspection showed relatively low caspase 3 and 7 activity indicating that minimal apoptosis is induced 
upon exposure to silica nanowires alone. This suggests that cell death due to nanowire inoculation occurs mostly because of necrotic processes, induced by physical displacement from the surface as opposed to apoptotic processes induced by cytotoxic effects of the nanowires. Our data revealed similar trends, showing an inert response to silica nanowires at $1 \mu \mathrm{g} \mathrm{ml}^{-1}$ concentrations for periods up to 48 hours (data not shown) with no negative effects on cellular proliferation monitored via the cellular impedance. Higher NW concentrations resulted in decreases in the cellular impedance (Fig. 13a) representing weaker tight junctions and cell adherence to the electrodes, eventually leading to total cell death within the first 24 hours of exposure. Our impedance based system was capable of real-time monitoring via multiple measurements of a single culture. This improves upon the temporal resolution available to procedures using standard assays, reducing the need for redundant samples.

$|\mathrm{Z}|$ of $50 \mu \mathrm{g} / \mathrm{ml}$ Sample

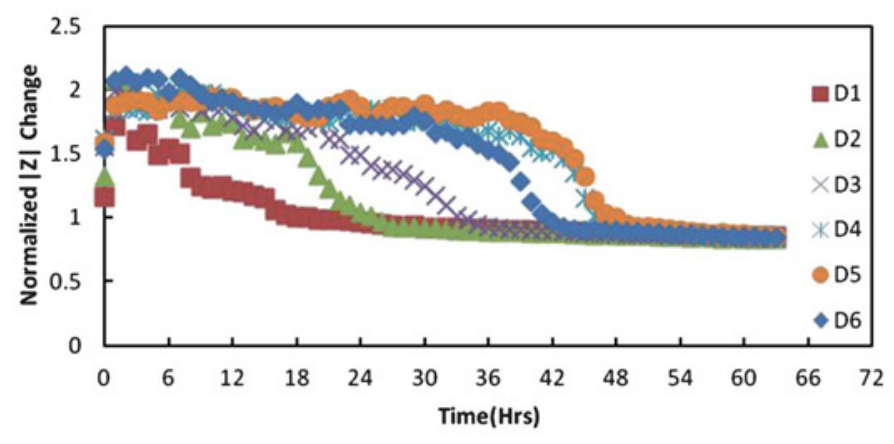

(a)

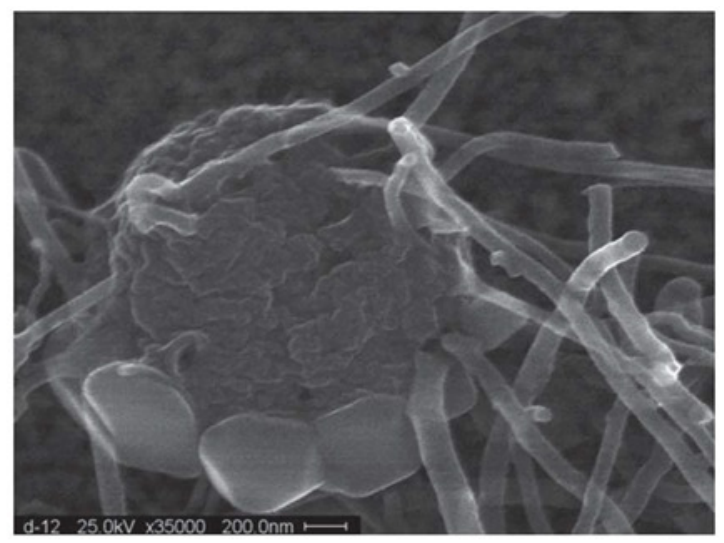

(b)

Figure X. (a) Impedance response of cells on a multiple electrodes on a device exposed to $50 \mathrm{mg} \mathrm{ml}^{-1}$ of silica nanowires. (b) SEM image revealing high density of nanowires causing cell death. (Reproduced with Permission[1]) 
In addition, the inertness of silica nanowires is further supported by the apparent spatial dependent response to nanowire inoculation at $50 \mu \mathrm{g} \mathrm{ml}^{-1}$. Fig. 13a shows that decreases in cellular impedance did not occur in concert. A sharp reduction in impedance is first seen at electrode D1 after six hours post NW-seeding. At 18 and 20 hours, the impedance at electrodes D2 and D3 drops significantly. Finally, at approximately 36 hours the impedance drops at the remaining electrodes indicating total cell death. The staggered effect on the impedance can be attributed to the random distribution of nanowires within suspension. Qi et al. examined the effect of high concentrations of nanowires on cellular adhesion by incubating HepG2 cells with $100 \mu \mathrm{g} \mathrm{ml}^{-1}$ of silica nanowires immediately after cellular seeding. $62.5 \%$ of cells were found to be adherent after 18 hours of incubation when compared with cells incubated alone. Scanning electron microscopy (SEM) and energy dispersive X-ray spectroscopy (EDS) found that rounded and poorly adherent cells had high levels of silicon surrounding them. Additionally, reverse transcriptase polymerase chain reaction (RT-PCR) found a down regulation in expression of adhesion specific proteins integrin, FAK, and Col I.

Our recorded impedance data confirms that areas exposed to larger amounts of NW died off faster than those in areas with lower amounts. After impedance measurements were complete, devices were imaged using SEM (Fig. 13b). It can be seen that areas surrounding electrodes contain a higher concentration of NWs which impeded cellular adhesion to the surface causing cell death. Furthermore, it was seen that cells can also be mechanically punctured by the NW. Finally, these results demonstrate a unique approach to studying the interactions between biomaterials and nanomaterials. By integrating a new electrode design, multi-spatial monitoring of a single cellular culture was realized. 


\section{Conclusion}

A new multi-spatial electrode design and real-time impedance monitoring system were used to demonstrate a potential application of monitoring the bio impedance of biological systems. Quantitative impedance measurements were used to verify prior experiments that found that lower concentrations of silica nanowires are inert when exposed to cellular cultures, evidenced by stable fluctuations in the measured impedance overtime. At concentrations of 50 and $100 \mu \mathrm{g} \mathrm{ml}^{-1}$ nanowires have fatal interactions due to mechanical disturbances that prevent proper adhesion to the surface. Due to the capability of this system to work in real-time, it improves upon the design of traditional experimental assays because it allows a higher number of time-points to be assessed without the need for increasing the number of experimental samples. Also, the use of an 8-electrode array enables multiple measurements of the same sample, adding spatial resolution and statistical value through measurement redundancy. By offering quantitative, real-time, and non-destructive measurements of nanowire-cell interactions, this system is a viable complement to standard endpoint assays.

\section{Chapter 6}

\section{Conclusions and Future Outlook}

Conclusions

Nanostructure integration into microscale sensing devices has been demonstrated using silica nanowires. The nanowires were integrated into an interdigitated microelectrode device without the use of commonplace nanowire transfer methods. A direct grown method was used to place nanowire into microscale device and was achieved without damaging the 
pre -existing sensing structure, by tailoring nanowire catalyst placement and synthesis atmosphere. Nanostructures formed using these approaches were uniformed in size and dimension with predictable morphologies and chemical compositions. Sensor platforms with nanowire structures were synthesized at $1050^{\circ} \mathrm{C}$ on $\mathrm{Si}<111>$ substrates and were processed in ambient environments without the use of hazardous precursors.

Silica nanowires were observed to determine how their physical and chemical properties could be tuned to develop devices, which could be mass produced with SERS substrate capabilities. Growth kinetic experiments performed using gold and palladium catalyst yielded dense and consistent silica nanowire growth. This consistent growth led to the development of growth model fitting, through use of the Maximum Likelihood Estimation (MLE) and Bayesian hierarchical modeling. It was determined from gathered data that an exponential-linear model was the best for fitting silica nanowire growth and was used to produce optimal nanowire growth patterns via Vapor Liquid Solid (VLS) growth methods. Further, the thermodynamic contributions of silicon and oxygen to the growth of silica nanowires was explored and used to explain growth factors that contributed to growth inhibition at temperatures higher that $1150^{\circ} \mathrm{C}$.

For potential use as in vivo diagnostic aid silica nanowires were examined in the cellular media containing epithelial breast cancer cells to determine the possible cytoxic affects. Quantitative studies were performed on this media using impedance spectroscopy, a technique used for studying cellular interactions in real time. It was found that palladium catalyzed silica nanowires were toxic to breast cancer cells, especially at higher concentrations. Cells seeded with 50 and $100 \mu \mathrm{g} / \mathrm{mL}$ of nanowires was prevented from proliferating a mechanical effect, as opposed to necrotic. However, over time (24 hours) the 
$1 \mu \mathrm{g} / \mathrm{mL}$ concentration of nanowires began causing mechanical disturbances in cellular interaction leading to cell death. This studied revealed that in lower concentration nanowires could be used as a diagnostic aid for short periods, which coincides with the current point of care objectives rapid and efficient sample testing and diagnostic correlation.

Additionally a method for direct nanowire integration was developed that allowed for silica nanowires to be grown directly into interdigitated micro-scale sensing structures. This technique eliminated the need for physical nanowire transfer thus preserving nanowire structure and performance integrity, which could lead to reduces fabrication cost. Successful nanowire integration was physical verified using Scanning Electron Microscopy and confirmed electrically using Electrochemical Impedance Spectroscopy of immobilized Prostate Specific Antigens (PSA).

Lastly, this work contributes to the use of nanostructures in unison with pre-existing micro-scale platforms as a gateway to improving drug delivery, point of care testing, and patient diagnosis. The experiments performed above serve as a guideline to addressing the metallurgic challenges in nanoscale integration of materials with varying composition and to understanding the effects of nanomaterials on biological structures that come in contact with the human body.

\section{Future Tasks}

Opportunities for further investigation past the tasks past the work presented are as follows but are limited to:

a) Enhance hydrogen gas sensing using Pd catalyzed silica nanowires.

Studies so far have shown that nanostructures can be used to detect/discriminate at the molecular level, but mass manufacturing of systems that contain them are non-existent. 
Silica nanowires are typically synthesized with $\mathrm{Pd}$ as the catalysts. This facilitates a sensing platform with high surface to volume ratio. Thereby, utilizing the nanowires for gas detection will yield enhanced and discriminatory response. To address this, data will be collected that pertains to, a) validating the nanowire gas detection mechanism thru biological particle mass transport, to verify sensing electrical viability, and b) studying the factors influencing the morphology of nanostructures, specifically tailoring nano-surfaces to insure reaction with gas particles.

b) Dielectric Barrier Layer Template/Polymer Temperature Characterization.

An alternative method for site-specific growth of silica nanowires on silicon wafers will be demonstrated using PECVD $\mathrm{SiO}_{2}$ dielectric barrier layer. The motivation for this work is to explicate the factors contributing to residual stress, deformation and fracture of PECVD silicon oxide films so as to obtain a quality film to be used as the DBL. PECVD oxide films are typically compressive and often cause convex wafer bowing.

The objective is to obtain a low tensile stress silicon oxide film by adjusting the relative concentration of the carrier gases in the plasma to control the film buckling and exploring others materials such nitride as replacement for $\mathrm{SiO}_{2}$ and a can be used for selective growth of silicon-based nanostructures (Figure1). Oxide film strength can be monitored using Stoney's equation to calculate the stress of the silicon oxide[87], where E is the Young's Modulus, $v$ is the Poisson Ratio, $t_{s}$ is the substrate thickness, $t_{\mathrm{f}}$ is the oxide thickness, and $R_{1}$ and $R_{2}$ is the wafer curvature of the bare silicon wafer and the deposited oxide layer respectively.

$$
S=\frac{E}{1-v} \cdot \frac{t_{s}^{2}}{6 t_{f}}\left(\frac{1}{R_{1}}-\frac{1}{R_{2}}\right)
$$



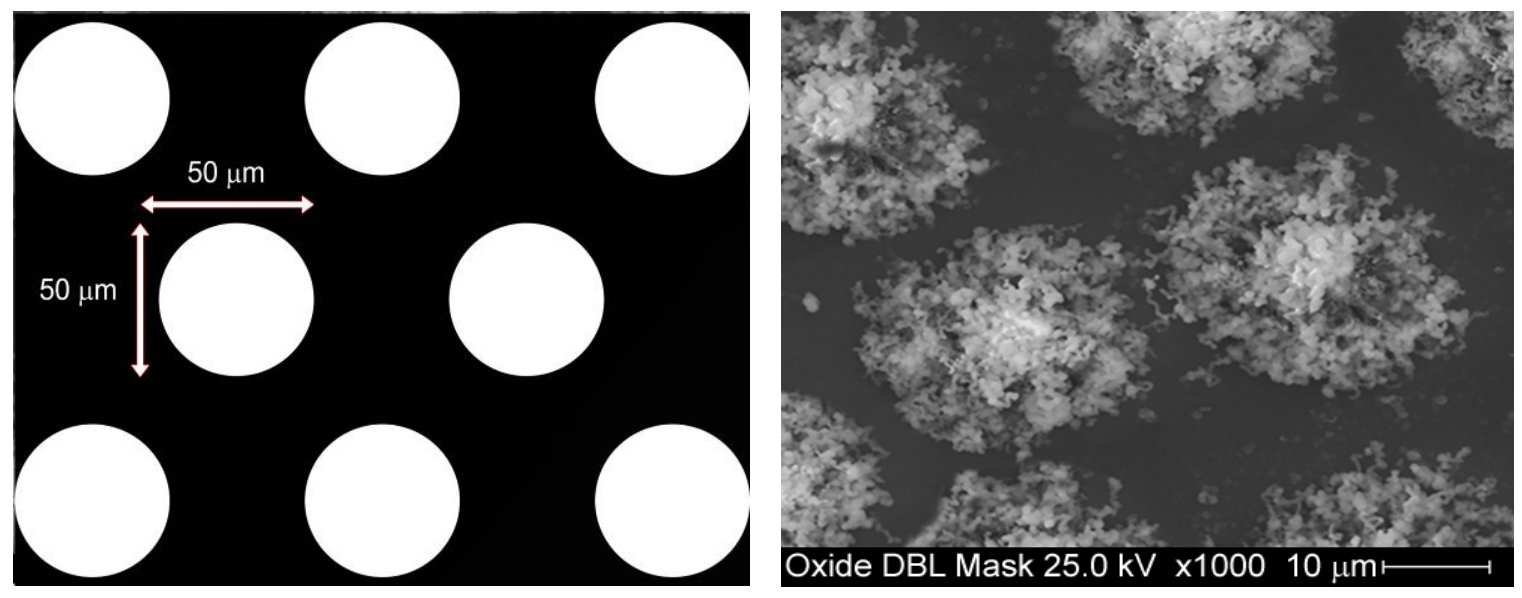

Figure 1. Nanowire growth restricted to by patterned DBL (a) Mask design of the a ideal Dielectric Barrier Layer system (b)SEM image of NW growth confined by an actual Dielectric Barrier Layer design.

c) Site specific placement of VLS catalyst for patterned nanowire growth

Additional understanding of metal catalyst is needed in order to grow nanostructures in patterned positions. Producing nanowires in such an ordered and with aligned positioning will aid in their use as detection mechanisms, by providing additional access via nanowire stem to analytes or other enzymatic agents. Most importantly, is the need for to exploration into the minimum catalyst drop size is needed to synthesize a single wire from a molten droplet. This data could be used in tandem with optimized growth conditions to fabricate a device structure that contains elements (nanowires) and is fully customizable, depending on sensing strategy or detection need (figure 2).

d) Copper was explored as nanowire growth catalysts. This material was specifically chosen for low temperature application. In work by Yao et. al [88] $\mathrm{Cu}$ was used a catalyst for low temperature synthesis of Si-based nanowires at $500^{\circ} \mathrm{C}$. The use of copper as a catalyst aligns well the use of Si-based nanowires due to the possibility of immediate integration into Si-based IC structures, as of current $\mathrm{Cu}$ is used an interconnect material. In the BioMEMS 
and Microsystems laboratory, nanowires with were produce using copper TEM grids and $\mathrm{Si}$ $<111>$ wafers at $300^{\circ}$ C. Figure 3 is a table top SEM micrograph showing the nanowires to vertically oriented moderate uniformity towards the middle of image. The tips of the wires are narrow and pointed with catalyst at tip. This is suggestive that the Vapor-Solid growth could be controlling the synthesis of these wires. These wires, with their low growth temperature and favorable morphologies have application in systems that involve the integration of nanostructures with microscale devices.

e) Lastly, differing growth substrates should be considered, for purpose of cost reduction and fabrication ease. Towards this in goal, graphene based nanostructures have been suggested and presented as new emerging technologies. Recently, Mohseni et. al [89] have produced vertically aligned high density single crystalline InAs nanowires graphene substrates using van der Waals epitaxy. This growth technique and substrate materials will make graphene based materials mainstream due to graphene's low cost and flexibility characteristics, a direct contrast to properties of silicon based materials.

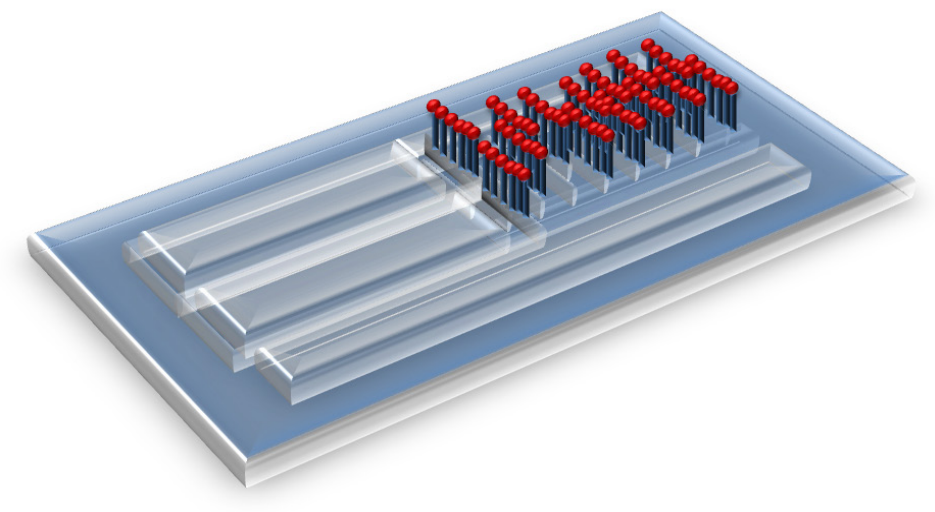

Figure 2. Ideal sensing device with vertically aligned silica nanowires integrated into interdigitated microelectrode structure. 


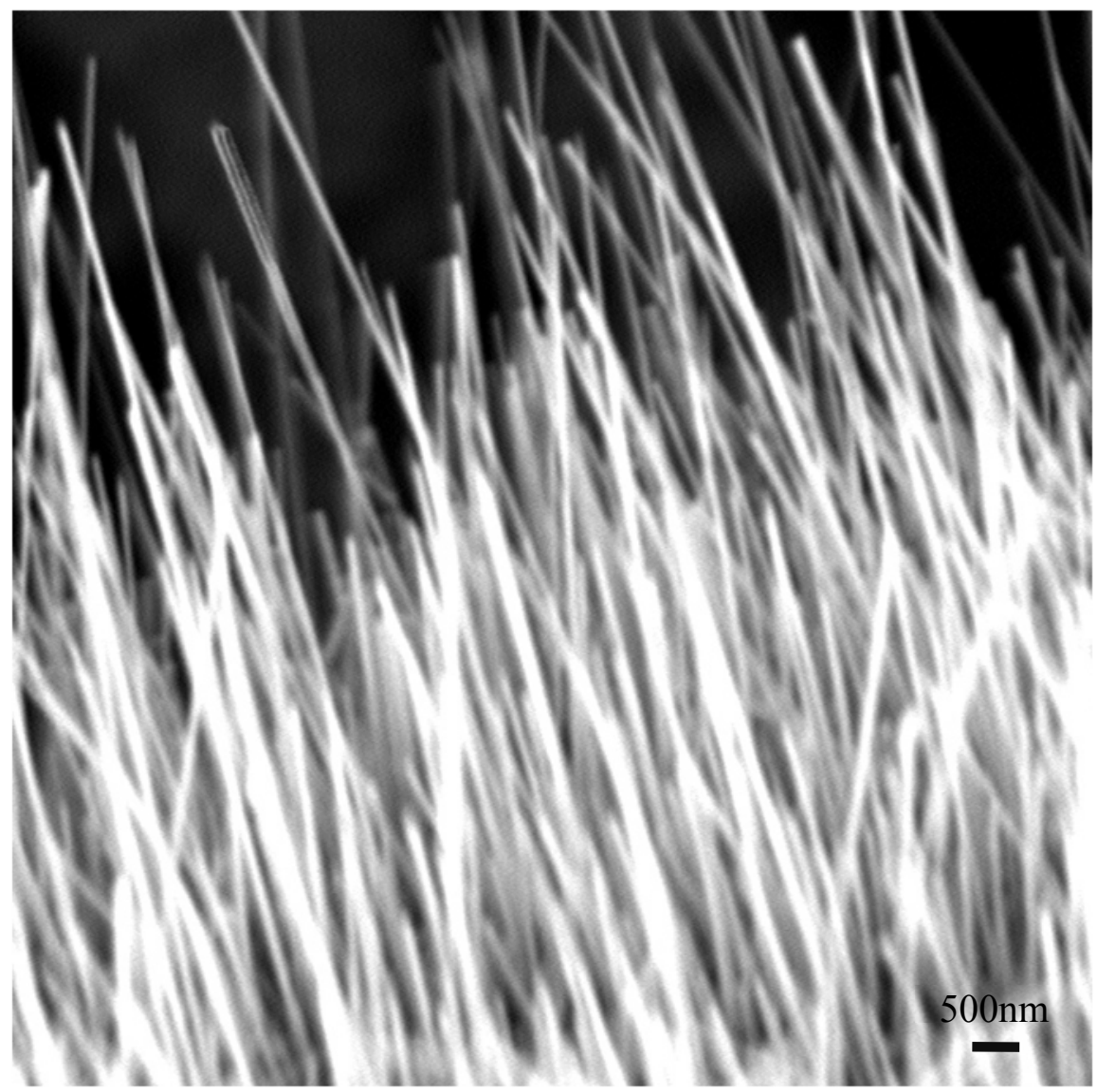

Figure 3. Nanowires grown from copper grid using silicon carrier wafer at $300^{\circ} \mathrm{C}$.[Courtesy Angela Colucci] 


\section{References}

1. Alexander Jr, F.A., et al., Real-time impedance analysis of silica nanowire toxicity on epithelial breast cancer cells. Analyst, 2012. 137(24): p. 5823-5828.

2. Huey, E., et al., Optimized growth and integration of silica nanowires into interdigitated microelectrode structures for biosensing. Sensors and Actuators B: Chemical, 2012. 175(0): p. 29-33.

3. Tong, L.G., R. R. Ashcom, J. B. He, S. Lou, J. Shen, M. Maxwell, I. Mazur, E. , Subwavelength-diameter silica wires for low-loss optical wave guiding. NATURE LONDON, 2003: p. 2.

4. Domachuk, P. and B.J. Eggleton, Photonics: Shrinking optical fibres. Nat Mater, 2004. 3(2): p. 85-86.

5. Alivisatos, P., The use of nanocrystals in biological detection. Nature biotechnology, 2004. 22(1): p. 47-52.

6. McAlpine, M.C., et al., Highly ordered nanowire arrays on plastic substrates for ultrasensitive flexible chemical sensors. Nat Mater, 2007. 6(5): p. 379-384.

7. McAlpine, M.C., R.S. Friedman, and C.M. Lieber, High-Performance Nanowire Electronics and Photonics and Nanoscale Patterning on Flexible Plastic Substrates. Proceedings of the IEEE, 2005. 93(7): p. 1357-1363.

8. Wagner, R.S. and W.C. Ellis, VAPOR-LIQUID-SOLID MECHANISM OF SINGLE CRYSTAL GROWTH. Vol. 4. 1964: AIP. 89-90.

9. Wu, Y. and P. Yang, Direct Observation of Vapor-Liquid-Solid Nanowire Growth. Journal of American Chemical Society, 2001. 123: p. 2.

10. Xu, J.M., Plastic electronics and future trends in microelectronics. Synthetic Metals, 2000. 115(1-3): p. 1-3.

11. R.F.Service, Electronics begin to flex their muscle. Science 2006(312): p. 1.

12. Reuss, R.H., et al., Macroelectronics: Perspectives on Technology and Applications. Proceedings of the IEEE, 2005. 93(7): p. 1239-1256.

13. Saito, R., G. Dresselhaus, and M.S. Dresselhaus, Physical properties of carbon nanotubes. Vol. 4. 1998: Imperial college press London.

14. Iijima, S., Helical microtubules of graphitic carbon. Nature, 1991/11/07. 354(6348).

15. Dresselhaus, M.S., et al., Carbon nanotubes. 2000: Springer. 
16. Meyyappan, M., A review of plasma enhanced chemical vapour deposition of carbon nanotubes. Journal of Physics D: Applied Physics, 2009. 42(21): p. 213001.

17. Levitt, A.P., Whisker technology. 1970: John Wiley \& Sons.

18. Liu, B. and H.C. Zeng, Hydrothermal Synthesis of ZnO Nanorods in the Diameter Regime of $50 \mathrm{~nm}$. Journal of the American Chemical Society, 2003. 125(15): p. 44304431.

19. Wang, J. and L. Gao, Hydrothermal synthesis and photoluminescence properties of $\mathrm{ZnO}$ nanowires. Solid State Communications, 2004. 132(3-4): p. 269-271.

20. Jiaqiang, X., et al., Hydrothermal synthesis and gas sensing characters of ZnO nanorods. Sensors and Actuators B: Chemical, 2006. 113(1): p. 526-531.

21. Liu, Z., et al., Synthesis of Copper Nanowires via a Complex-Surfactant-Assisted Hydrothermal Reduction Process. The Journal of Physical Chemistry B, 2003. 107(46): p. 12658-12661.

22. Lin, L.W., et al., Water-assisted synthesis of silicon oxide nanowires under supercritically hydrothermal conditions. Journal of Applied Physics, 2007. 101(1): p. 014314-7.

23. Xie, B.-Q., et al., A Hydrothermal Reduction Route to Single-Crystalline Hexagonal Cobalt Nanowires. European Journal of Inorganic Chemistry, 2006. 2006(12): p. 2454-2459.

24. Wang, X. and Y. Li, Selected-Control Hydrothermal Synthesis of $\alpha$ - and $\beta$-MnO2 Single Crystal Nanowires. Journal of the American Chemical Society, 2002. 124(12): p. 2880-2881.

25. Wang, Z., et al., A Simple Hydrothermal Route to Large-Scale Synthesis of Uniform Silver Nanowires. Chemistry - A European Journal, 2005. 11(1): p. 160-163.

26. Jaeger, R.C., G.W. Neudeck, and R.F. Pierret, Introduction to Microelectronic Fabrication, 2002, Prentice Hall.

27. Zhang, R.Q., Y. Lifshitz, and S.T. Lee, Oxide-Assisted Growth of Semiconducting Nanowires. Advanced Materials, 2003. 15(7-8): p. 635-640.

28. Shi, W.S., et al., Oxide-assisted growth and optical characterization of galliumarsenide nanowires. Applied Physics Letters, 2001. 78(21): p. 3304-3306.

29. Okamoto, H. and T.B. Massalski, The Au-Si (Gold-Silicon) system. Bulletin of Alloy Phase Diagrams, 1983. 4(2): p. 190-198.

30. Okamoto, H., Pd-Si (Palladium-Silicon). Journal of Phase Equilibria and Diffusion, 2007. 28(2): p. 231-232. 
31. Tanner, L. and H. Okamoto, The Pt-Si (Platinum-Silicon) System. Journal of Phase Equilibria, 1991. 12(5): p. 571-574.

32. Wacaser, B.A., et al., Preferential interface nucleation: an expansion of the VLS growth mechanism for nanowires. Advanced Materials, 2009. 21(2): p. 153-165.

33. Wang, H., et al., Morphology Control in the Vapor-Liquid-Solid Growth of SiC Nanowires. Crystal Growth \& Design, 2008. 8(11): p. 3893-3896.

34. Kim, H.W.S., S. H. Lee, J. W. Lee, C. Hwang, H. J. Chung, S.-Y. Kim, H.-S. Hwang, S. K. Yeom, G. Y. Lee, N.-E., Synthesis, Structural Characterization, and Photoluminescence Properties of SiO x Nanowires Prepared Using a Palladium Catalyst. JOURNAL- KOREAN PHYSICAL SOCIETY, 2007. 50(6).

35. Hu, J.Q., et al., A simple large-scale synthesis of very long aligned silica nanowires. Chemical Physics Letters, 2003. 367(3-4): p. 339-343.

36. Morales, A.M. and C.M. Lieber, A Laser Ablation Method for the Synthesis of Crystalline Semiconductor Nanowires. Science, 1998. 279(5348): p. 208-211.

37. Chang, P.-C., et al., ZnO Nanowires Synthesized by Vapor Trapping CVD Method. Chemistry of Materials, 2004. 16(24): p. 5133-5137.

38. Truhlar, D.G., Electron Scattering. Semiemperical Methods of Electronic Structure Calculations, Part B: Applications, 1977. 8.

39. Praveen Kumar Sekhar, S.N.S., Dinesh K Sood and Shekhar Bhansali, Selective growth of silica nanowires in silicon catalysed by Pt thin film Nanotechnology, 2006. 17(18).

40. Sarkar, J., G. Khan, and A. Basumallick, Nanowires: properties, applications and synthesis via porous anodic aluminium oxide template. Bulletin of Materials Science, 2007. 30(3): p. 271-290.

41. Heath, J.R., Superlattice Nanowire Pattern Transfer (SNAP). Accounts of Chemical Research, 2008. 41(12): p. 1609-1617.

42. Lee, K.-N., et al., Well controlled assembly of silicon nanowires by nanowire transfer method. Nanotechnology, 2007. 18(44): p. 445302.

43. Heath, J.R. and M.A. Ratner, Molecular electronics. 2003.

44. Melosh, N.A., et al., Ultrahigh-density nanowire lattices and circuits. Science, 2003. 300(5616): p. 112-115.

45. Jones, T.B., Basic theory of dielectrophoresis and electrorotation. Engineering in Medicine and Biology Magazine, IEEE, 2003. 22(6): p. 33-42. 
46. Hughes, M.P., AC electrokinetics: applications for nanotechnology. Nanotechnology, 2000. 11(2): p. 124.

47. Close, G.F., et al., A $1 \mathrm{GHz}$ integrated circuit with carbon nanotube interconnects and silicon transistors. Nano Letters, 2008. 8(2): p. 706-709.

48. Agarwal, V., et al. A CMOS integrated thermal sensor based on Single-Walled Carbon Nanotubes. in Sensors, 2008 IEEE. 2008: IEEE.

49. MacNaughton, S., et al. Carbon nanotube and graphene based gas micro-sensors fabricated by dielectrophoresis on silicon. in Sensors, 2010 IEEE. 2010: IEEE.

50. Morgan, H. and N.G. Green, AC electrokinetics: colloids and nanoparticles. 2003: Research Studies Press.

51. Pohl, H.A. and I. Hawk, Separation of living and dead cells by dielectrophoresis. Science, 1966. 152(3722): p. 647-649.

52. Chen, C.-L., et al., The heterogeneous integration of single-walled carbon nanotubes onto complementary metal oxide semiconductor circuitry for sensing applications. Nanotechnology, 2009. 20(22): p. 225302.

53. Lucci, M., et al., Gas sensing using single wall carbon nanotubes ordered with dielectrophoresis. Sensors and Actuators B: Chemical, 2005. 111: p. 181-186.

54. Dan, Y., et al., Dielectrophoretically assembled polymer nanowires for gas sensing. Sensors and Actuators B: Chemical, 2007. 125(1): p. 55-59.

55. Smith, P.A., et al., Electric-field assisted assembly and alignment of metallic nanowires. Applied Physics Letters, 2000. 77(9): p. 1399-1401.

56. Martin, C.R., Membrane-based synthesis of nanomaterials. Chemistry of Materials, 1996. 8(8): p. 1739-1746.

57. Hernández, R.M., et al., Template fabrication of protein-functionalized goldpolypyrrole-gold segmented nanowires. Chemistry of Materials, 2004. 16(18): p. 3431-3438.

58. John F. Conley, J., L. Stecker, and Y. Ono, Directed integration of ZnO nanobridge devices on a Si substrate. Applied Physics Letters, 2005. 87(22): p. 223114.

59. Saif Islam, M., et al., A novel interconnection technique for manufacturing nanowire devices. Applied Physics A, 2005. 80(6): p. 1133-1140.

60. Islam, M.S., et al., Ultrahigh-density silicon nanobridges formed between two vertical silicon surfaces. Nanotechnology, 2004. 15(5): p. L5. 
61. Tabib-Azar, M., et al., Mechanical properties of self-welded silicon nanobridges. Applied Physics Letters, 2005. 87(11): p. 113102-113102-3.

62. Hochbaum, A.I., et al., Controlled growth of Si nanowire arrays for device integration. Nano Letters, 2005. 5(3): p. 457-460.

63. Tufts, R. PANALYTICAL X'PERT PRO MRD. 2009 [cited 2013 June 1]; Available from:

http://nnrc.eng.usf.edu/documents/tools/Panalytical\%20Xpert\%20Pro\%20MRD.pdf.

64. Beane, R.J., Using the Scanning Electron Microscope for Discovery Based Learning in Undergraduate Courses. Journal of Geoscience Education, 2004. 52(3): p. 250253.

65. Bieber, J. Hitachi S-800 Scanning Electron Microscope. 2009 [cited 2013 June 01]; Available from: http://nnrc.eng.usf.edu/documents/tools/Hitachi\%20S800\%20Scanning\%20Electron\%20Microscope.pdf.

66. Yusuf Emirov, P.D. TRANSMISSION ELECTRON MICROSCOPE TECNAI F20. 2009 [cited 2013 June 1]; Available from:

http://nnrc.eng.usf.edu/documents/tools/Transmission\%20Electron\%20Microscope $\%$ 20TECNAI\%20F20.pdf.

67. Wang, J., Electrochemical biosensors: Towards point-of-care cancer diagnostics. Biosensors and Bioelectronics, 2006. 21(10): p. 1887-1892.

68. Arya, S.K., et al., Advances in materials for room temperature hydrogen sensors. Analyst, 2012. 137(12): p. 2743-2756.

69. A.Kishore Reddy, B.S.R., P.Prathyusha, T.Anirudh bhargav, T.Rajani,V.Rajamanickam, P.Krishna Reddy, M.Arul selvan, Emerging Trends of Nanotechnology in Cancer Therapy, in International Journal of Pharmaceutical \& Biological Archives. 2011. p. 8.

70. Ferrari, M., Cancer nanotechnology: opportunities and challenges. Nat Rev Cancer, 2005. 5(3): p. 161-171.

71. Woodward Iv, J.T. and J.A. Zasadzinski, Thermodynamic limitations on the resolution obtainable with metal replicas. Journal of Microscopy, 1996. 184(3): p. 157-162.

72. Yao, Y., F. Li, and S.-T. Lee, Oriented silicon nanowires on silicon substrates from oxide-assisted growth and gold catalysts. Chemical Physics Letters, 2005. 406(4-6): p. 381-385.

73. Suzuki, T., Effect of annealing a silicon wafer in argon with a very low oxygen partial pressure Journal of Applied Physics, 2000. 88(11): p. 6. 
74. Sekhar, P.K. and S. Bhansali, Manufacturing aspects of oxide nanowires. Materials Letters, 2010. 64(6): p. 729-732.

75. Yang, Y., et al., The effect of annealing temperature, residual O2 partial pressure, and ambient flow rate on the growth of SiOx nanowires. Applied Physics A: Materials Science \& Processing, 2012. 107(4): p. 885-890.

76. Kim, T.-H., A. Shalav, and R.G. Elliman, Active-oxidation of Si as the source of vapor-phase reactants in the growth of $\mathrm{SiO}[\mathrm{sub} x]$ nanowires on $\mathrm{Si}$. Journal of Applied Physics, 2010. 108(7): p. 076102.

77. Min, J. and A.J. Baeumner, Characterization and optimization of interdigitated ultramicroelectrode arrays as electrochemical biosensor transducers. Electroanalysis, 2004. 16(9): p. 724-729.

78. Mamishev, A.V., et al., Interdigital sensors and transducers. Proceedings of the IEEE, 2004. 92(5): p. 808-845.

79. Alexander Jr, F., D.T. Price, and S. Bhansali. Optimization of interdigitated electrode (IDE) arrays for impedance based evaluation of Hs $578 T$ cancer cells. in Journal of Physics: Conference Series. 2010: IOP Publishing.

80. Arya, S.K., et al., Antibody functionalized interdigitated [small mu ]-electrode (ID[small mu ]E) based impedimetric cortisol biosensor. Analyst, 2010. 135(8): p. 1941-1946.

81. Murphy-Perez, E., S.K. Arya, and S. Bhansali, Vapor-liquid-solid grown silica nanowire based electrochemical glucose biosensor. Analyst, 2011. 136(8): p. 16861689.

82. Schwan, H.P., Electric characteristics of tissues. Radiation and Environmental Biophysics, 1963. 1(3): p. 198-208.

83. Schwan, H.P., Electrical properties of tissue and cell suspensions. Advances in biological and medical physics, 1957. 5: p. 147.

84. Giaever, I. and C.R. Keese, Micromotion of mammalian cells measured electrically. Proceedings of the National Academy of Sciences, 1991. 88(17): p. 7896-7900.

85. Qi, S., et al., Effects of Silicon Nanowires on HepG2 Cell Adhesion and Spreading. ChemBioChem, 2007. 8(10): p. 1115-1118.

86. Julien, D.C., et al., In vitro proliferating cell models to study cytotoxicity of silica nanowires. Nanomedicine: Nanotechnology, Biology and Medicine, 2010. 6(1): p. 84-92. 
87. Mackenzie, K.D., et al. Characterization \& Optimization of Low Stress PECVD Silicon Nitride for Production GaAs Manufacturing. in The International Conference on Compound semiconductor manufacturing technology. 2004.

88. Yao, Y. and S. Fan, Si nanowires synthesized with $\mathrm{Cu}$ catalyst. Materials Letters, 2007. 61(1): p. 177-181. 


\title{
Appendix
}

\section{Physical Knowledge Integration in Nano-manufacturing using Approximate Bayesian Computation}

Li Wang $^{1}$, Qiang Huang ${ }^{1^{*}}$, Eric Huey ${ }^{2}$, Subramanian Krishnan $^{2}$, Shekhar Bhansali ${ }^{3}$

${ }^{1}$ Daniel J. Epstein Department of Industrial and Systems Engineering University of Southern California, Los Angeles, California, 90089, United States

${ }^{2}$ Department of Electrical Engineering, University of South Florida, Tampa, Florida, 33620, United States

${ }^{3}$ Department of Electrical and Computer Engineering, Florida International University, Miami, FL, 33174, United States

\begin{abstract}
There is plenty of modeling work from a physical perspective in nano-manufacturing. Due to their deterministic nature, however, they are inadequate in characterization of nanomanufacturing processes as large variability is generally observed. The stochastic nature of processes involved in nano-manufacturing means opportunities for statistical modelling. The challenge in integrating existing physical knowledge in such statistical models is that physical knowledge is often presented in differential equation form which may have no closed form solution and thus yields difficulties in determination of likelihood function. Another issue is that the observations are often with large noises. In this study, we propose a method that naturally integrates physical knowledge with statistical model by using approximate Bayesian computation $(\mathrm{ABC})$ method. In this approach, the use of likelihood function can be avoided and we can estimate the parameters in the differential equation form physical models directly. Numerical simulation as well as a case study based on silica nanowire growth would be included to show the performance of the proposed approach.
\end{abstract}




\section{INTRODUCTION}

Nano-manufacturing is the controllable manipulation of materials structures, components, devices, and systems at the nanoscale (1 to 100 nanometers) in one, two, and three dimensions for large-scale reproducibility of value-added components and devices [1]. It is the essential step in transferring the advances in nanotechnology into commercial production and achieving their potential. Currently, however, there are still great challenges facing nano-manufacturing as the typical yield of nano-devices is $10 \%$ or less[2]. Process modeling will provide the needed understanding and is of great importance in order to obtain improvement. The current physical models, however, cannot describe the real process satisfactorily as there is large variability [3-4]. In this study, we will propose a novel way based on Approximate Bayesian computation (ABC) method to integrate such physical knowledge with statistical models which has large potential in characterizing nanomanufacturing processes.

Specifically, we will use Si nanowire growth process as an example. The advantages of studying the Si nanowire growth is that there are large amount of physical knowledge available[5-9].Those physical models, however, due to the difficulties in obtaining closed form solution and large variability in experiment observations, cannot be directly applied. This makes this process be a good candidate to try our new approach.

In section 2, we first introduce some existing physical model for Si nanowire growth. Approximate Bayesian computation method will be introduced in section 3 followed by simulation study and a case study based on actual silica nanowire growth data. 


\section{PhysicAl Model for Si NANOWIRE GROWTH}

Since vapor-liquid-solid (VLS) mechanism been introduced for the whisker growth in 1964 by Wagner and Ellis[5], there are two kinds of models been developed, namely, absorption-induced[5-7] and diffusion-induced models[7-9]. Ruth and Hirth[8] proposed a NW growth rate model:

*Corresponding author: Tel.: (213) 740-7433; Fax: (213) 740-1120; Email: qiang.huang@usc.edu

$$
\begin{aligned}
& \frac{\mathrm{dL}}{\mathrm{dt}}=\frac{2 \Omega\left(\mathrm{N}_{\infty}-\mathrm{N}_{0}\right) \mathrm{L}}{\tau \mathrm{R}} \text {, if NW length } \mathrm{L} \leq \mathrm{L}_{\mathrm{f}}=\sqrt{2 \mathrm{D} \tau} \text {, as } \mathrm{L}_{\mathrm{f}} \text { is the diffusion length } \\
& \frac{\mathrm{dL}}{\mathrm{dt}}=\frac{2 \mathrm{~L}_{\mathrm{f}} \Omega\left(\mathrm{J}-\mathrm{N}_{0} / \tau\right)}{\mathrm{R}} \text {, if } \mathrm{L}>\mathrm{L}_{\mathrm{f}}
\end{aligned}
$$

where $\mathrm{D}$ is diffusion coefficient, $\tau$ is mean life time of adatom on the NW wall, $\Omega$ is atomic volume of $\mathrm{Si}$, $\mathrm{J}$ is impingement flux, $\mathrm{R}$ is $\mathrm{NW}$ radius, $\mathrm{N}_{0}$ is adatom concentration at the liquid alloy, and $\mathrm{N}_{\infty}$ is adatom concentration at the base substrate. For more complicated molecular beam epitaxy, Dubrovskii et.al[5] developed a model to unify two types of VLS models. Those physical models, presenting in differential equation forms, often do not have closed form solution and thus present a challenge for building statistical model based on them as there is no closed formed maximum likelihood function. Another issue is that in nano-manufacturing, data is often limited as the sampling procedures are both time consuming and expensive. As a result, the maximum likelihood estimator, which based on the asymptotic behavior of the samples, could be inappropriate. In [3-4], Huang et.al proposed to use Bayesian framework to avoid those problems, but those work are limited 
with model structures which have an explicit functional dependence between response and input variables and cannot be directly applied in the case when the dependence is in a differential equation form as we discussed.

\section{APPROXIMATE BAYESIAN COMPUTATION}

For Bayesian parameter estimation setting, we have a likelihood function $\mathrm{f}(\mathrm{x} \mid \theta)$, prior distribution $\pi(\theta)$ on the parameter space $\Theta$. With those information, we can obtain the posterior distribution of $\theta$ by computing $f(\theta \mid x) \propto f(x \mid \theta) \pi(\theta)$ given the observed $x$. The difficulty lies in the fact that for the nano-manufacturing, the physical knowledge often presents in an ordinary, partial or stochastic differential equation form and a closed form solution can be difficult to obtain or even nonexistent.

Approximate Bayesian computation makes use of simulation in performing statistical inference in this setting[10]. Due to its ability to deal with normally analytically or computationally intractable complex systems, it is widely used in estimation in biological systems[11-14]. In [11], $\mathrm{ABC}$ is used to estimate the parameters in models describing complicated Y chromosome microsatellite mutation process. Beaumont et.al [12] argues that the $\mathrm{ABC}$ method combines many advantages of Bayesian inferences as well as high computational efficiency when summary statistics are used instead of entire observed data set. Markov Monte Carlo method has also been proposed to be integrated into $\mathrm{ABC}$ to improve acceptance rate and further improve computational efficiency [13]. The $\mathrm{ABC}$ method, however, also has some limitations due to the potential information loss due to the use of summary statistics and the evaluation of approximated posterior distribution [13]. For 
our study, as we choose to use all observed data instead of summary statistics, the information loss issue is avoided and the adequacy of approximated posterior distribution's adequacy can be checked by the estimation result.

The key idea of $\mathrm{ABC}$ is to replace likelihood function evaluation by parameter simulation from prior distribution. For example, if our model is that:

$$
\frac{d y}{d x}=g(x \mid \theta), y_{0}=a, \text { with } X=\left(x_{1}, \ldots, x_{d}\right)
$$

Our objective is to estimate $\theta$ based on noisy observation $Y^{\prime}=Y+\varepsilon, \varepsilon \sim N_{d}\left(0, \sigma^{2} I_{d}\right)$ as $Y=\left(y_{1}, \ldots, y_{j}, \ldots, y_{m}\right)$ corresponding to $X_{j}$ values. The difficulty with standard Bayesian estimation procedure is that it may be impossible to integrate $\mathrm{g}(\mathrm{x} \mid \theta)$ analytically and thus we cannot get an analytical expression of likelihood function $f(x \mid \theta)$. In this case, with $A B C$ rejection algorithm, we simply generate $\theta^{*}$ from $\pi(\theta)$, then we can numerically obtain $\mathrm{y}^{*}$ using the differential equation, initial condition and a normal random generator for each $\mathrm{x}$. With a distance norm $\rho$, if $\rho\left(\mathrm{Y}^{*}, \mathrm{Y}^{\prime}\right) \leq \mathrm{s}$, which is a specified tolerance level, then we accepted $\theta^{*}$. Repeat the process $n$ times, we obtain an approximate sample from the posterior distribution. As one may observe, when $s \rightarrow 0$, the approximate posterior distribution becomes exact. For continuous random variables, however, the tolerance level s cannot be 0 and a small s would yield an approximated posterior distribution.

To improve the approximation, Beaumont et.al [12] proposed an improvement that considered the distance between each sample with the observation. The basic idea is that the distribution of sample point which is closer to the observation is closer to the exact posterior distribution. In order to implement the idea, two procedures are used: 
1) Weighting $\theta_{i}$ based on $\rho\left(Y^{*}-Y^{\prime}\right)$

2) Adjusting $\theta_{i}$ using local linear regression

In this study, we will adopt this improved version of $\mathrm{ABC}$ proposed by Beaumont et.al[13] as it allows us to increase the tolerance level $s$ to increase the $\theta^{*}$ acceptance rate while the approximation is insensitive to the choice of s. Euclidean norm is chosen for the distance norm $\rho\left(\mathrm{Y}^{*}, \mathrm{Y}^{\prime}\right)=\sqrt{\sum_{\mathrm{j}=1}^{\mathrm{m}}\left(\mathrm{y}_{\mathrm{j}}^{*}-\mathrm{y}_{\mathrm{j}}^{\prime}\right)^{2}}$ and Epanechnikov kernel $\mathrm{K}_{\mathrm{S}}(\mathrm{t})$ is chosen for weighting:

$$
\mathrm{K}_{\mathrm{s}}(\mathrm{t})= \begin{cases}\operatorname{cs}^{-1}\left(1-\left(\frac{\mathrm{t}}{\mathrm{s}}\right)^{2}\right) & , \mathrm{t} \leq \mathrm{s} \\ 0 & , \mathrm{t}>\mathrm{s}\end{cases}
$$

while $\mathrm{c}$ is a normalizing constant.

\section{SiMULATION STUDY}

To demonstrate the applicability of the improved $\mathrm{ABC}$ method and investigate the effect of choice of tolerance level s, a simulation study is performed. The physical model behind of the simulation is from [15] and is used to describe the InAs nanowire length growth:

$$
\frac{\mathrm{dl}}{\mathrm{dt}}=\mathrm{V}_{\mathrm{L}} \tanh \left(\frac{\sqrt{2} \mathrm{l}}{\lambda_{\mathrm{NW}}}\right)
$$

Here $\mathrm{l}$ is the length of nanowire, $\mathrm{t}$ is the growth time, $\mathrm{V}_{\mathrm{L}}$ is the linear growth rate and $\lambda_{\mathrm{NW}}$ is the diffusion length of the material at the temperature. 
For the simulation study, we first simulate $20(\mathrm{l}, \mathrm{t})$ pairs with known parameter $\mathrm{V}_{\mathrm{L}}$ and $\lambda_{\mathrm{NW}}$ using numerical integration, then add a small normal noise to the simulated length to simulate the inherent uncertainty in the growth process as well as the observation error. The parameter setting for the simulation is:

$$
\mathrm{V}_{\mathrm{L}}=10, \frac{\sqrt{2}}{\lambda_{\mathrm{NW}}}=0.01, \mathrm{l}(1)=0.05
$$

The noise added is normally distributed with mean 0 and $\sqrt{2}$ standard deviation. One exception is that the length should not be smaller than 0 , so when the random noise makes the length smaller than 0 , we replace it with 0.01 . The simulated data set is listed in Table A1 in appendix.

With the $\mathrm{ABC}$ procedure introduced in previous section, we set the prior distribution of $\pi\left(\mathrm{V}_{\mathrm{L}}\right) \sim \mathrm{U}(0,20)$ and $\pi\left(\frac{\sqrt{2}}{\lambda_{\mathrm{NW}}}\right) \sim \mathrm{U}(0,0.02)$ as diffused and non-informative. The tolerance level s is set as 300,400 and 800 and we do 100,000 runs.

The estimation result is summarized in Table 1 and 2. As the tolerance level becomes larger, the number of acceptance becomes larger, but the estimation result becomes more biased with larger variation due to less accurate approximation of true posterior distribution. Figure 1 also shows that with smaller tolerance level, the estimated curve converges to the true curve(using mean of accepted parameters). Figure 2 shows an example of approximate posterior distribution of $\mathrm{V}_{\mathrm{L}}$ converges to a symmetrical distribution with mean close to true mean under 300 tolerance level. 
Table 1: Acceptance rate with tolerance level

\begin{tabular}{l|l}
\hline Tolerance Level & Number of \\
& Acceptance \\
\hline 300 & 71 \\
500 & 158 \\
800 & 272 \\
\hline
\end{tabular}

Table 2: Posterior Distribution of Parameters

\begin{tabular}{l|l|l|l|l|l}
\hline Tolerance & Parameter & 25 Percentile & Median & 75 Percentile & Standard \\
& & & & & Deviation \\
\hline 300 & $\mathrm{~V}_{\mathrm{L}}$ & 9.992 & 10.064 & 10.140 & 0.13 \\
& $\sqrt{2} / \lambda_{\mathrm{NW}}$ & 0.01004 & 0.01010 & 0.01018 & 0.0001 \\
\hline 500 & $\mathrm{~V}_{\mathrm{L}}$ & 10.016 & 10.091 & 10.189 & 0.16 \\
& $\sqrt{2} / \lambda_{\mathrm{NW}}$ & 0.01005 & 0.01013 & 0.01022 & 0.0002 \\
\hline 800 & $\mathrm{~V}_{\mathrm{L}}$ & 10.048 & 10.141 & 10.263 & 0.19 \\
& $\sqrt{2} / \lambda_{\mathrm{NW}}$ & 0.01004 & 0.01014 & 0.01028 & 0.0002 \\
\hline
\end{tabular}



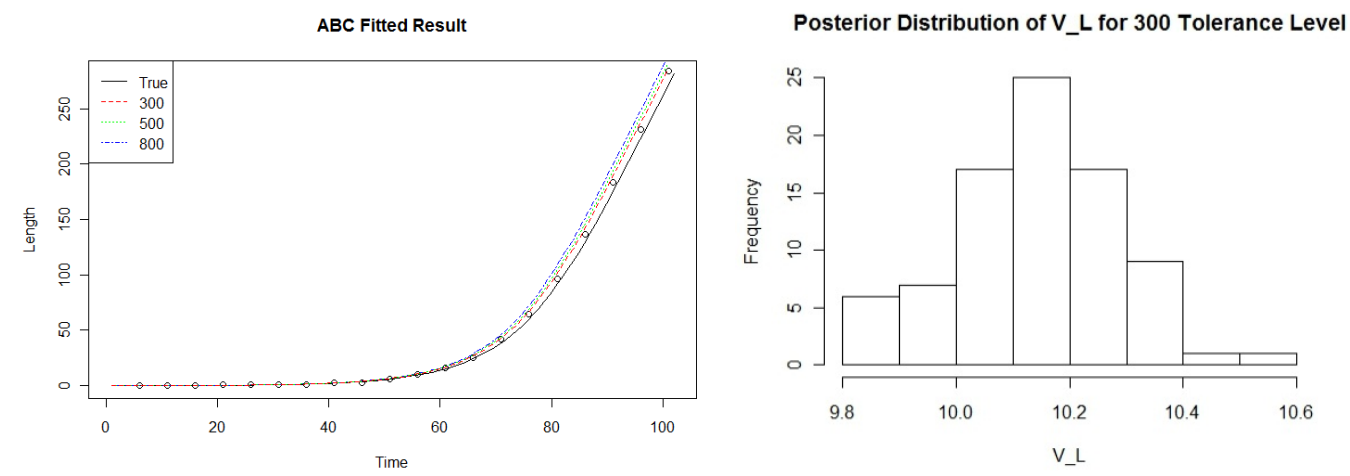

Figure 1: Comparison of different tolerance level Figure 2: Posterior distribution of $\mathrm{V}_{\mathrm{L}}$ under 300 tolerance level

\section{CASE Study}

In [4], we explored the weight growth of silica nanowire catalyzed by $\mathrm{Pd}$ under 1100 degrees Celsius. In this case study, we performed experiments under another temperature setting, namely, 1050 degrees Celsius and tried to see if the weight growth can be modeled in the physical model in [15], which is used in previous section. The data is listed in Table 2.

Table 2: Weight Growth under 1050 degrees Celsius

\begin{tabular}{l|l|l|l}
\hline Weight $(\mathrm{mg})$ & Time(min) & Weight $(\mathrm{mg})$ & Time $(\mathrm{min})$ \\
\hline 0.000 & 5 & 0.160 & 75 \\
0.000 & 5 & 0.331 & 75 \\
0.009 & 5 & 0.240 & 90 \\
0.005 & 5 & 0.321 & 90 \\
0.016 & 10 & 0.240 & 90 \\
\hline
\end{tabular}




\begin{tabular}{l|l|l|l}
\hline 0.078 & 10 & 0.210 & 96 \\
0.060 & 20 & 0.240 & 96 \\
0.044 & 20 & 0.528 & 120 \\
0.086 & 20 & 0.443 & 120 \\
0.050 & 30 & 0.282 & 180 \\
0.114 & 30 & 0.344 & 180 \\
0.161 & 30 & 0.753 & 220 \\
0.040 & 40 & 0.832 & 220 \\
0.120 & 60 & 0.700 & 240 \\
0.090 & 60 & 0.663 & 240 \\
0.195 & 60 & & \\
\hline
\end{tabular}

With $\pi\left(V_{\mathrm{L}}\right) \sim \mathrm{U}(0,0.4)$ and $\pi\left(\frac{\sqrt{2}}{\lambda_{\mathrm{NW}}}\right) \sim \mathrm{U}(0,0.1)$ based on observation on the data. The tolerance level s is set as 4.75 and we do 100,000 runs. The estimation result is summarized in Table 3. The fitting result and posterior parameter distribution for $\mathrm{V}_{\mathrm{L}}$ can be seen in Figure 3 and 4. As one can observe, the fitting result is reasonable give large variations observed in data.

Table 3: Case Study Summary

\begin{tabular}{l|l|l|l|l|l|l}
\hline Tolerance & Number of & Parameter & 25 & Median & 75 & Standard \\
Level & Acceptance & & Percentile & & Percentile & Deviation \\
\hline
\end{tabular}




\begin{tabular}{l|l|l|l|l|l|l}
\hline 4.75 & 2197 & $\mathrm{~V}_{\mathrm{L}}$ & 0.17 & 0.22 & 0.31 & 0.09 \\
& & $\sqrt{2} / \lambda_{\mathrm{NW}}$ & 0.031 & 0.046 & 0.067 & 0.02 \\
\hline
\end{tabular}
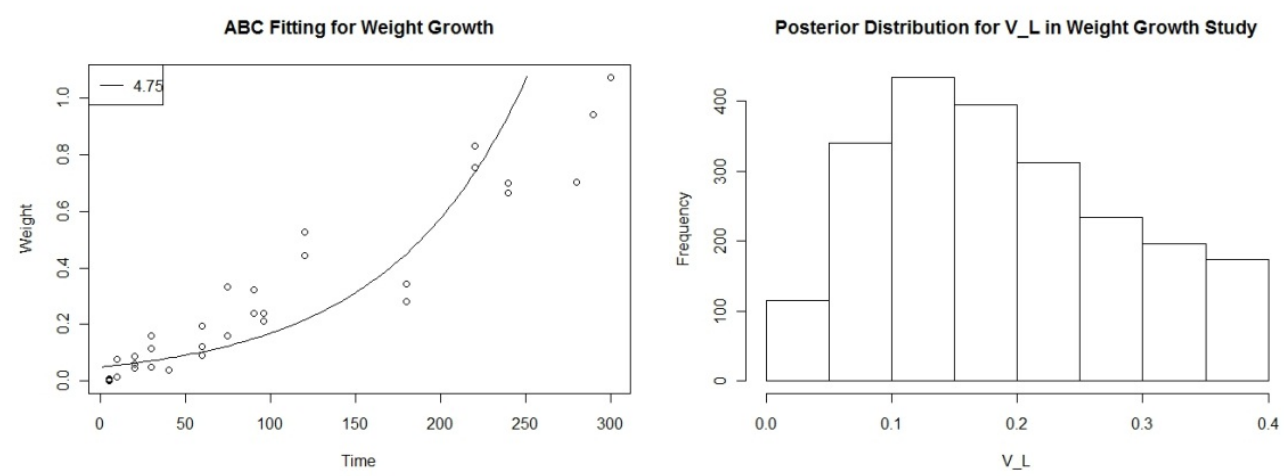

Figure 3: $\mathrm{ABC}$ fitting result for weight growth Figure 2: Posterior distribution of $\mathrm{V}_{\mathrm{L}}$ for weight growth study

\section{SUMMARY}

In summary, this paper proposed using $\mathrm{ABC}$ algorithm to handle the problem associated with physical differential equation models in nano-manufacturing, namely, the maximum likelihood function is unavailable. To show the applicability of our proposed method, both simulation study and real world data is presented.

\section{ACKNOWLEDGEMENTS}

This work is partially supported by NSF grants CMMI-1002580 and NSF CMMI-0700659. 


\section{REFERENCES}

[1] http://www.internano.org

[2] Kuo, W., "Challenges related to reliability in nano-electronics", IEEE Transactions on Reliability, Vol.55, No.4, pp.569-570, 2006.

[3] Huang, Q.,"Physics-Driven Bayesian Hierarchical Modeling of Nanowire Growth Process at Each Scale", IIE Transactions, IIE Transactions on Quality and Reliability, Vol. 43, pp. 1-11,2011.

[4] Huang, Q., Wang, L., Dasgupta, T., Zhu, L., Sekhar, P.K., and, Bhansali, S., An, Y. "Statistical Weight Kinetics Modeling for Silica Nanowires Growth Catalyzed by Pd Thin Film", IEEE Trans on Automation Science and Engineering, Vol. 8, pp.303310,2011 .

[5] Wagner,R.S. and Ellis, W.C.:" Vapor-Liquid-Solid Mechanism of Single Crystal Growth", Applied Physics Letters, Vol.4, pp.89-90, 1964

[6] Givargizov,E. , "Fundamental aspects of VLS growth", J. Crystal Growth, Vol. 31, pp. $20-30,1975$.

[7] V. G. Dubrovskii, N. V. Sibirev, G. E. Girlin, J. C. Harmand, and V.M. Ustinov, "Theoretical analysis of the vapor-liquid-solid mechanism of nanowire growth during molecular beam epitaxy", Phys. Rev. E, Vol.73, p. 021603, 2006.

[8] V. Ruth and J. P. Hirth, "Kinetics of diffusion-controlled whisker growth", J. Chem. Phys., Vol. 41, no. 10, pp. 3139-3149, 1964.

[9] Kikkawa, J., Ohno, Y., and Takeda,S. "Growth rate of silicon nanowires", Appl. Phys. Lett., Vol. 4, p. 123109, 2005

[10] Tavare, S., Balding, D.J., Griffiths, R.C., and Donnelly, P." Inferring Coalescence Times from DNA Sequence Data", Genetics, Vol. 2, pp, 505-518, 1997

[11] Pritchard, J.K. and Seielstad, M.T. and Perez-Lezaun, A. and Feldman, M.W., "Population growth of human Y chromosomes: a study of Y chromosome microsatellites", Molecular Biology and Evolution,Vol.16, no.12, pp.1791-1798, 1999

[12] Beaumont, M.A. and Zhang, W. and Balding, D.J.," Approximate Bayesian computation in population genetics", Genetics,Vol.162, no.4, pp.2025,2002

[13] Marjoram, P. and Molitor, J. and Plagnol, V. and Tavare, S.,"Markov chain Monte Carlo without likelihoods", PNẢ S, Vol.100,no.26,p.15324, 2003

[14] Beaumont, M.A, Cornuet, J., Marin, J. and Rober, C, "Adaptive approximate Bayesian computation", Biometrika, Vol. 96, pp. 983-990, 2009.

[15] Dayeh, S.A., Yu, E. T and Wang D., "Surface Diffusion and Substrate-Nanowire Adatom Exchange in InAs Nanowire Growth", Nano Letters, Vol.9, pp. 1967-1972, 2009. 
APPENDIX

Table A1: Simulated Growth Data

\begin{tabular}{l|l|l|l}
\hline Length $(\mathrm{nm})$ & Time $(\mathrm{min})$ & Length $(\mathrm{nm})$ & Time(min) \\
\hline 0.01 & 6 & 10.02 & 56 \\
0.26 & 11 & 16.12 & 61 \\
0.13 & 16 & 25.58 & 66 \\
1.23 & 21 & 41.84 & 71 \\
0.67 & 26 & 64.35 & 76 \\
1.21 & 31 & 96.55 & 81 \\
0.87 & 36 & 136.84 & 86 \\
3.06 & 41 & 183.79 & 91 \\
2.97 & 46 & 231.08 & 96 \\
5.78 & 51 & 284.14 & 101 \\
\hline
\end{tabular}

Nanowire separation and Sorting Studies

One of the main challenges in this work was the complication in growing nanowires directly at the desired device. With the current processes, nanowires are removed from growth substrate via mechanical scrapping motion and the fragments are placed onto desired device. This technique is physically damaging to the nanowires. Therefore, a more expedient way to transfer nanowires has become extremely vital.

In current process explored below figure $1 \mathrm{a}-\mathrm{d}$, nanowires are scrapped from the silicon chip and are placed in a test tube. The white film covering the chip exhibits the presence of nanowires. More dense is the white layer, more are the nanowires. To ensure that no nanowires are lost, we then put $1 \mathrm{~mm}$ of acetone solution into the test tube where the scrapped nanowires were place, from the walls. The acetone solution turns slightly white 
acknowledging the presence of nanowires. Now in order to separate the nanowires, which are usually clustered, we place that test tube in the sonication machine.

In sonication, the electrical signal is converted into physical vibrations by the use of piezoelectric crystal. The vibration helps to separate nanowires from its clusters by an effect known as cavitation. Cavitation is a process which occurs due to the vibration, where large amount of microscopic bubbles are formed and destroyed simultaneously. When the bubbles burst, they send small shockwaves to the nanowires. Collapsing of thousands of these bubbles create a relatively powerful effect to disunite the nanowires.
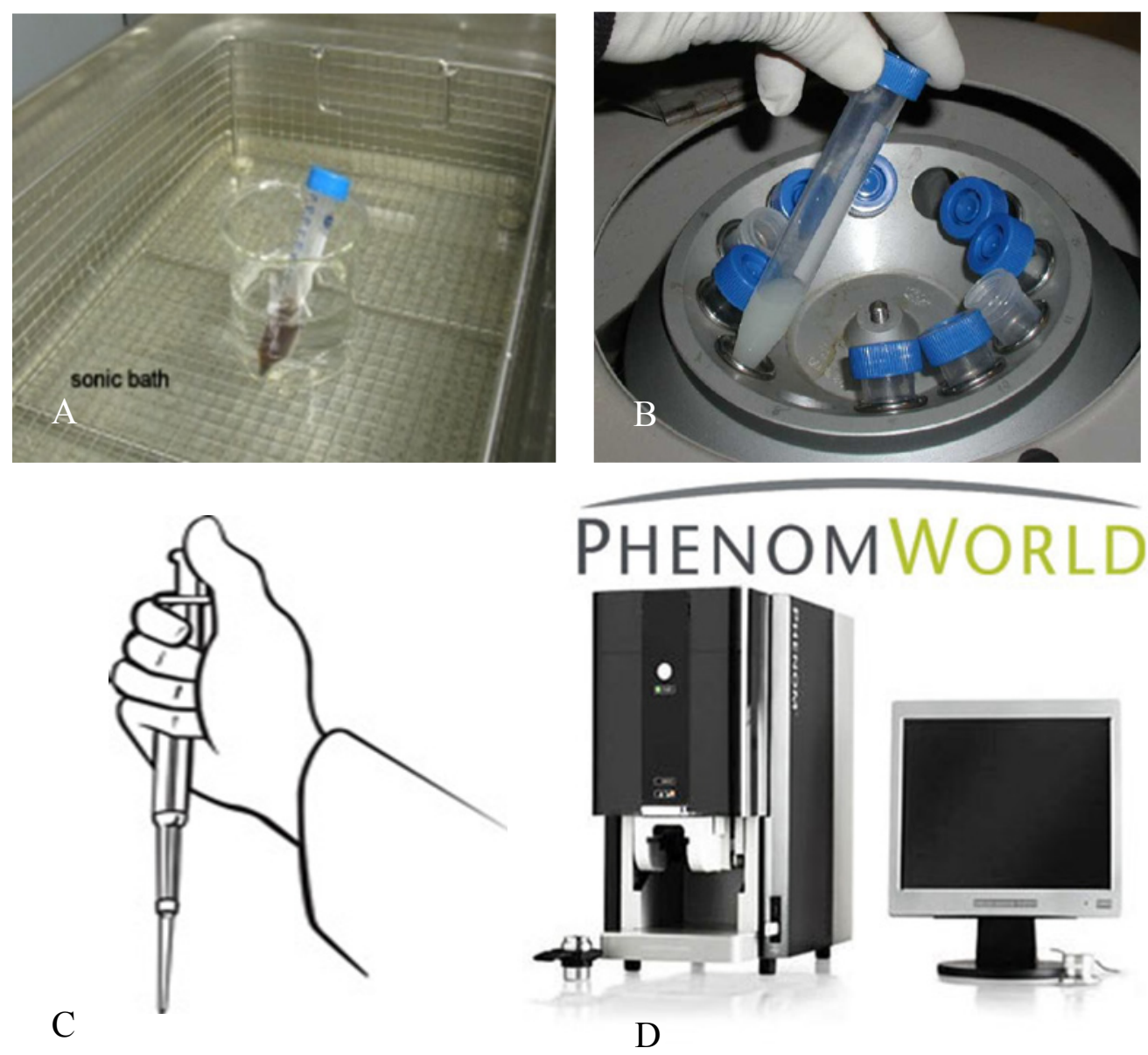

Figure 1. Process steps for nanowire separation study, a) sonic bath, b) centrifuge, c) pipette solution dropper, and d) imaging device 


\section{Observations}

Figures 2 are micrographs of samples where the nanowires were removed from growth substrate. Nanowires did latch onto TEM grids as well as expected. Groups of nanowires were found to be similarly sized. During TEM operation nanowires reacted to the electron beam and would burn away or drift out of view after prolonged exposure. Nanowires did adhere together; this is attributed to the natural positive and negative charge build-up along the oxide wire surface. Scraping the nanowires over the small test tube was difficult and may have caused introductions of artifacts observed in figure $2 \mathrm{a}$. In the future, before centrifuging a vicious oil-based substance could be used to coat the nanowires to reduce charge build up along the surface to aid in separation.
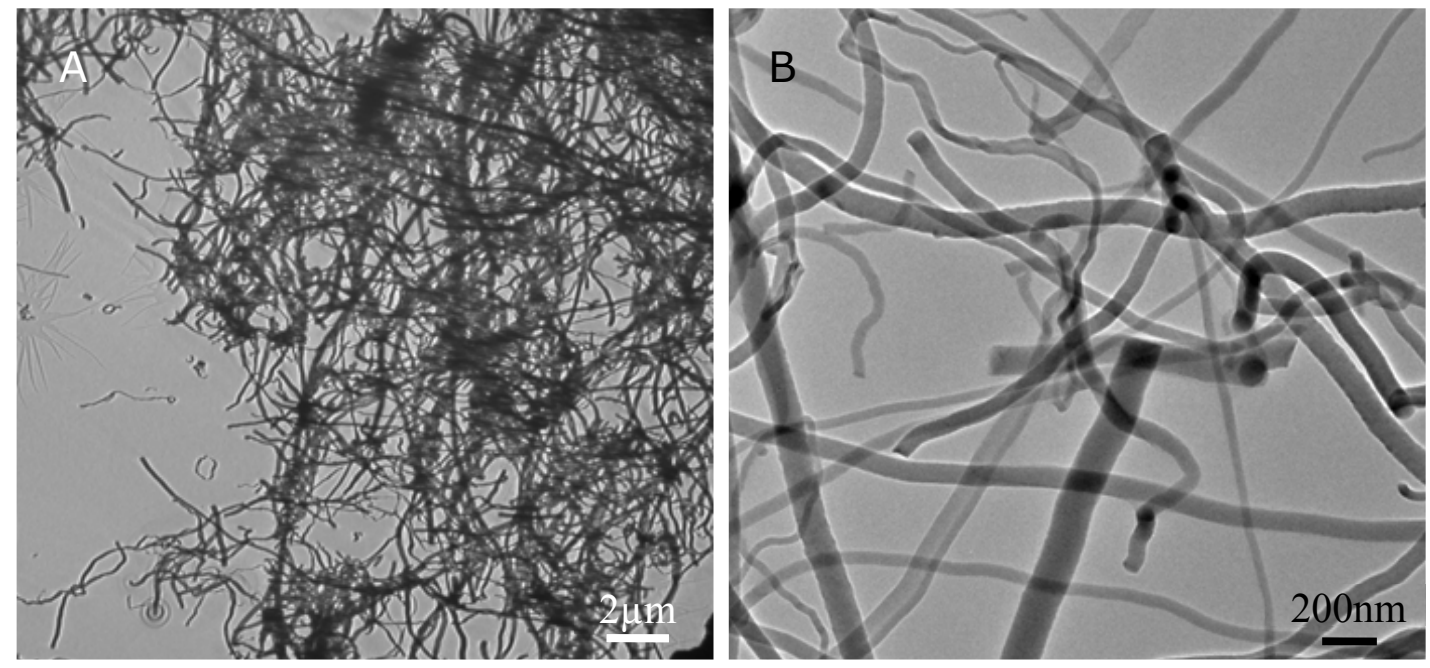

Figure 2. TEM images of scraped and sorted silica nanowires dispersed in Acetone. A) 2um and B) 200nm resolution's. 


\section{VITA}

\section{ERIC G. HUEY}

\section{Summary}

Published nanoscience researcher with seven years of experience working with engineering and bio-science. Interested in an opportunity that will allow for engagement and interaction with other knowledgeable, motivated, and achievement driven individuals in a collaborative setting.

\section{Education}

Doctorate of Philosophy, Electrical Engineering GPA: 3.67

Florida International University, Miami, FL

Master of Science, Electrical Engineering GPA: 3.67

University of South Florida, Tampa, FL

Bachelor of Science, Electrical Engineering, GPA 2.81

Southern University and A\&M College, Baton Rouge, LA

Experience

National Synchrotron Light Source, Brookhaven National Laboratory

Upton, New York

$2006 \& 2009-2012$

Summer Research Scientist

- Design/Built Atomic Layer Deposition System

- Cost Savings: $\$ 200,000$

- Design/Fabricated MOS Capacitors with Hafnium Oxide\Aluminum Contacts

- Published Results in Applied Physics Letters

○ Appl. Phys. Lett. 97, 242108 (2010); doi:10.1063/1.3524262

- Assisted in Strategy Development for Maia Detector (Fabrication/Characterization)

○ Maia Detector won DOE 2011 R\&D Award

\section{Research Experience for Undergraduates (REU) at Michigan State University}

East Lansing, Michigan

June 2008- August 2008

Research Intern

- Learned Thin Film Deposition Techniques (Sputtering, Evaporation, Atomic Layer Deposition)

- Co-Authored First Study on Electrical Scattering and Spin-Flipping at Metallic Interfaces

○ DOI:10.1103/PhysRevB.82.220401 


\section{Publications}

Eric Huey, Subramanian Krishnan, Brandon Richard, Sylvia Thomas, Shekhar Bhansali, "Growth Characteristics of Pd Catalyzed Silica Nanowires. Materials Research Letters, 2013. Submitted

B. Richard, N. Bolden, E. Huey, J. Lee, and S. Thomas, "Optical Effects of Doping Levels of Cobalt-doped ATO Sol-Gels on Carbon Fibers for Reflective Composite Coatings" Under Preparation

Alexander Jr, F.A.* Huey, Eric G., Price, Dorielle T., Bhansali, Shekhar, "Real-time impedance analysis of silica nanowire toxicity on epithelial breast cancer cells". Analyst, 2012. 137(24): p. 5823-5828.

*Joint First Author

Eric Huey, Sunil K. Arya, Subramanian Krishnan, Abhishek Dey, Shekhar Bhansali, "Optimized growth and integration of silica nanowires into interdigitated microelectrode structures for biosensing" Sensors and Actuators B: Chemical, Dec. 2011 Doi:10.1016/j.snb.2011.11.056

Hoang Yen Thi Nguyen, R. Acharyya, E. Huey, B. Richard, et al. "Conduction Electron Scattering and Spin-Flipping at Sputtered Co/Ni Interfaces", American Physical Society 2010, Phys. Rev. B 82, 220401(R) (2010)DOI:10.1103/PhysRevB.82.220401

Abdul K. Rumaiz, J. C. Woicik, G. A. Carini, D. P. Siddons, E. Cockayne, E. Huey, P. S. Lysaght, D. A. Fischer, and V. Genova, "Band alignment of atomic layer deposited $\mathrm{HfO}_{2}$ on clean and N passivated germanium surfaces”, Appl. Phys. Lett. 97, 242108 (2010);

DOI:10.1063/1.3524262

Huey, Eric, Siddons, D.P., "Development of Single and Double GEM (Gas Electron Multiplier) Based Detectors", Department of Energy, Journal of Undergraduate Research vol. VII, 2007

\section{Skills/Software}

OSHA 30-hour Certificate in General Industry Standards, Bench testing and oscilloscope, Photolithography, Metrology,PSPICE, Simulation, Labview, AutoCAD, Ansoft, Microsoft Office, MATlab, multimeters and protoboards 